*wals View/Frint Document Cover Sheet

This document was retrieved from the Boeing ISEARCH System.

Accession \#: D196071710

Document \#: SD-WM-DP-105

Title/Desc:

FINAL REPORT FOR TANK 241B112 AUGER SAMPLES 95-AUG-014 \& 95-AUG-015 


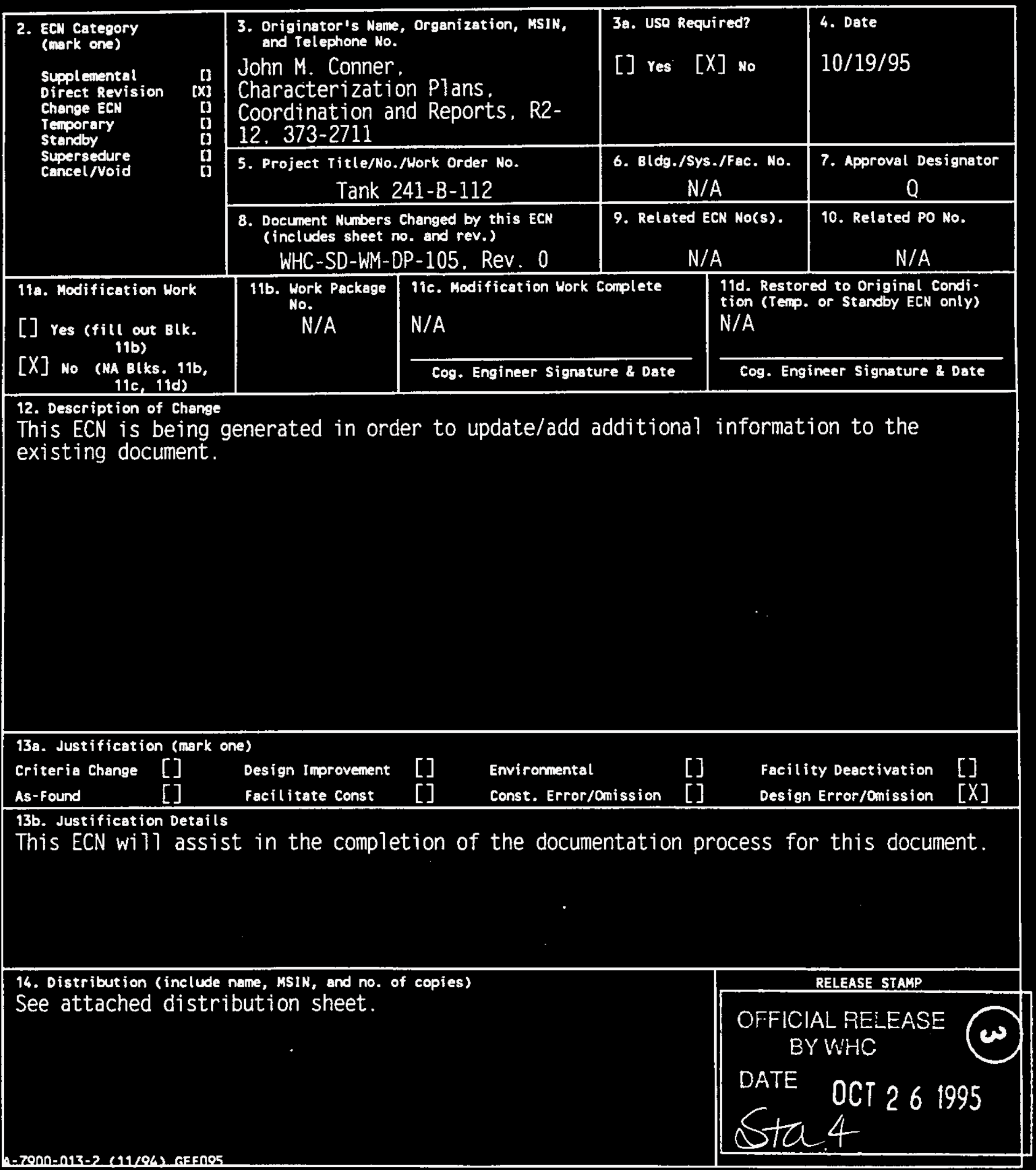




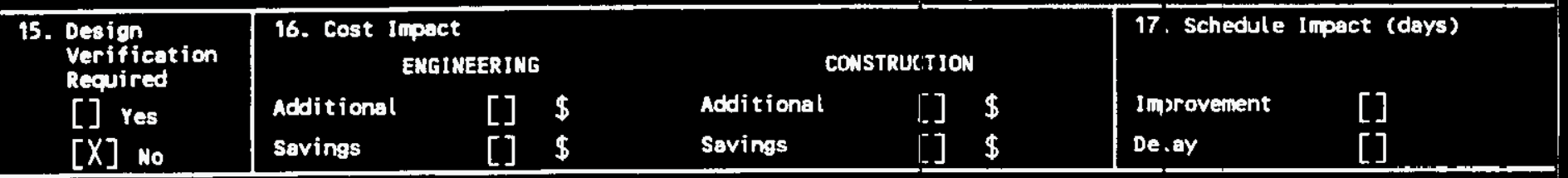

18. Change Inpact Review: Indicate the related docuirents (other than the engineer ing docunents ident if ied on side 1) that will be affected by the change described in Block 12 . Enter the affected document number in Block 19.

\section{SDD/DD}

Functional Design Criteris

Operating Specification

Criticality Specitication

Conceptual Design Report

Equipment Spec.

Const. Spac.

Procurement Spec.

Vendor Information

OM Manus!

FSAR/SAR

Sotety Equipmant List

Radiation Work Permit

Environmental Impact Statement

Environmental Report

Environmantal Permit
[]
[]
[]
[]
[]
[]
[]
[]
[]
[]
[]
[]
[]
[]
[]
[]
[]
[]
[]
[]
[]
[]
[]
[]
[]
[]
[]
[]
[]
[] Tank Calibration Manual Health Physics Procedure Stress/Design Report Interface Contral Drawing Calibration Procedure Installation Procedure

Maintenance Procedure Engingoring Procedure Operating Instruction Operating Proceduro Operational Safoty Requirement IEFD Drawing

Coll Arrangament Drawing Essential Material Specification Fac. Proc. Samp. Schedule Inspaction Plan Inventory Adjustmont Request Spares Multiple Unit Listing Test Procedures/Specification Component Index ASME Coded hem

[] Humen Factor Consideration

[] Computer Software

Electric Circuit Schedule

ICRS Procedure

19. Other Affected Documents: (NOTE: Documents listed belou will not be revised by this ECW.) Signatures below indicate that the signing organization has been notified of other affected documents $\mathrm{l}$ isted below. Document Nuber/Revision

N/A

Process Control Manual/Plan

[] Process Flow Chart

Purchase Roquisition

Ticklor File

]

20. Approvals

\section{signature}

OPERATIONS AND E,YAIMEERING

Cog. Eng. J.M. Comer

Cog. Mgr. J.G. Kristof zski

M/hir

aA Tzu L. Tung Tom

Environ.

other

program Support
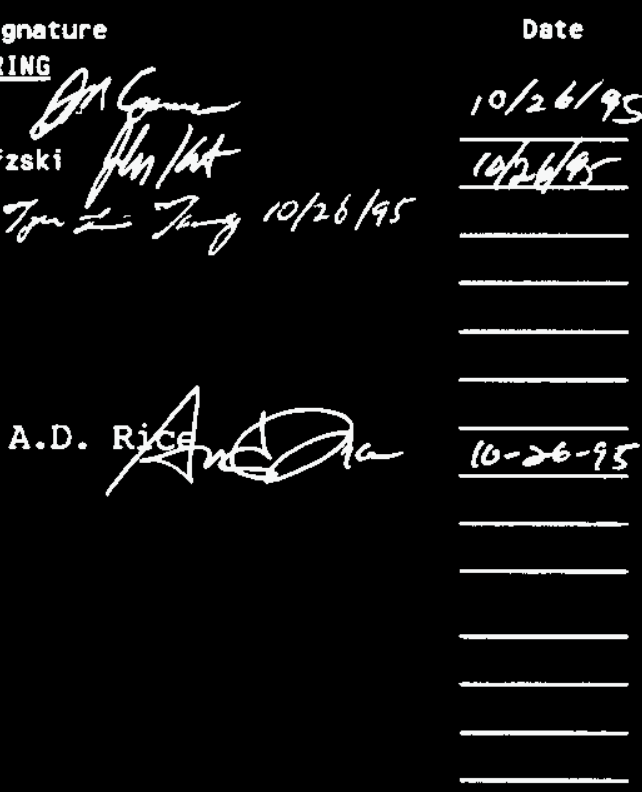

Signature

Date

\section{ARCHITECT-ENGIWEER}

PE

DA

Safety

Design

Environ.

other

\section{DEPARTMERT OF ENERGY}

signature or a Control Number that tracks the Approval signature

\section{ADDI TIOHAL}




\section{RELEASE AUTHORIZATION}

Document Number: WHC-SD-NM-DP-105, REV I

Documant Titte:

Final Report for Tank 241-B-112, Auger Samples 95AUG-014 and 95-AUG-015

Release Date:

$10 / 26 / 95$

This document was reviewed following the procedures described in WHC-CM-3-4 and is:

APPROVED FOR PUBLIC RELEASE

WHC Information Release Administration Specialist:
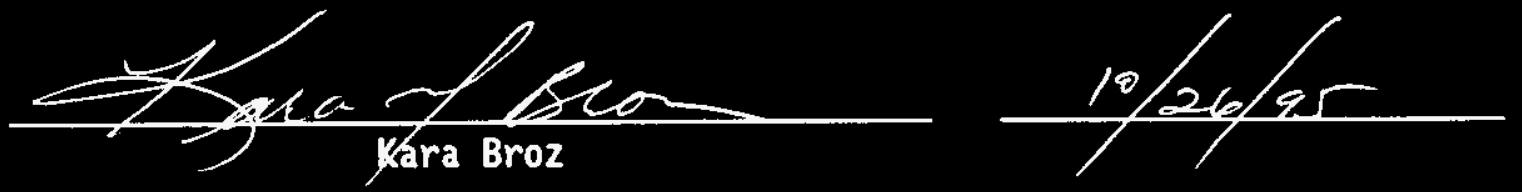

TRADEMARK DISCLATWER. Reference herein to any specific commercial product, process, or service by trade name, trademark, manufacturer, or otherwise, does not necessarily constitute or imply its endorsenent, recommendation, or favoring by the United States Government or any agency thereof or its contractors or subcontractors.

This report has been reproduced from the best available copy. Available in paper copy. Printed in the United States of America. To obtain copies of this report, contact:

Westinghouse Hanford Company - Document Control Services

P.0. Box 1970, Mailstop H6-08, Richland, WA 99352

Telephone: (509) 372-2420; Fax: (509) 376-4989 
THS PAGE INTENTIONALUY IEET BLANK 
2. Title

Final Report for Tank 241-B-112, Auger Samples 95AUG-014 and 95-AUG-015

5. Key Mords

Final Report, Tank 241-B-112, Tank B-112, B-112, B Farm. Auger Samples, 95-AUG-014, 95-AUG-015
3. Number

WHC-SD-WM-DP-105

4. Rev No. 1

6. Author

Name: John M. Conner

signatuf Come

Organization/Charge code $75310 /$ N4G4C

\section{Abstract}

N/A

8.

RELEASE STAMP

OFFICIAL RELEASE

BY WHC

DATE IOCT ? \& 1035

sta 4 
HIS PAGE INTENTIONALUY

LEFT BLANK 
(2) Title

Final Report for Tank 241-B-112. Auger Samples 95-AUG-014 and 95-AUG-015

CHANGE CONTROL RECORD

(3) Revision 0

(7) Initialiy released $05 / 01 / 95$ on EDT610421.
Authorized for Release

(5) Cog. Engr. (6) Cog. Mar. Date

J.M. Conner J.G. Kristofzski

J.M. Conner J.G. Kristofzski

1 RS Incorporate per ECN-625764. 

MHC-SD-MIN-DP-105, REV. 1

AMALYTICAL SERVICES

FINAL REPORT FOR TANK 241-B-112, AUGER SAMPLES 95-AUG-014 AND 95-AUG-015

Date Printed:

OCTOBER 18, 1995 


\section{TS PAGE INTENTIONAULY THIS PAGET BLANK}


MHC-SD-NIM-DP-105, REV. 1

TABLE OF CONTENTS

PART I Final Report for Tank 241-B-112, Auger Samples 95-AUG-014 and 95AUG-015

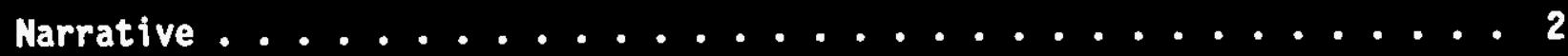

Chain of Custody Forms .................. 4

Hot Cell Logbook ..................... 7

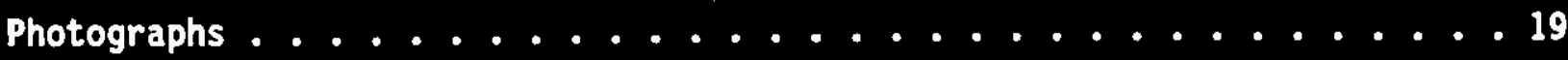

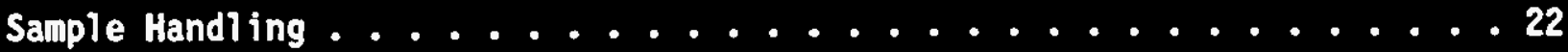

Extrusion Worklist $\$ 862 \ldots \ldots 23$

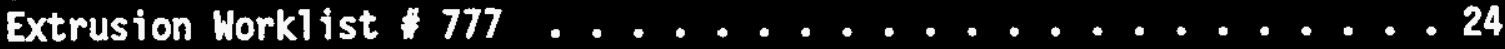

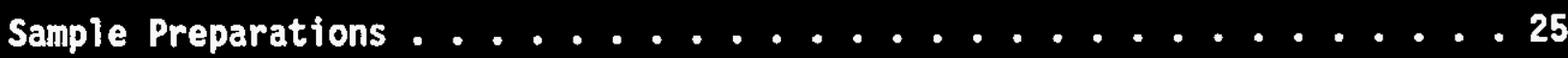

Fusion Digestion Workl ist $\$ 997 \ldots . . . . . . . . .26$

Fusion Digestion Worklist $11192 \ldots . . . . . . . .27$

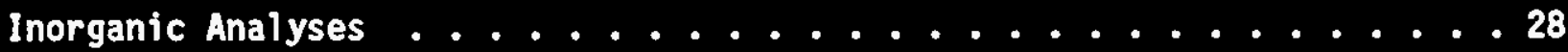

Differential Scanning Calorimetry (DSC)

DSC Morklist *1207. . . . . . . . . . . . . . 29

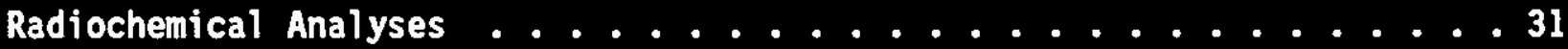

Alpha Total Analysis (AT)

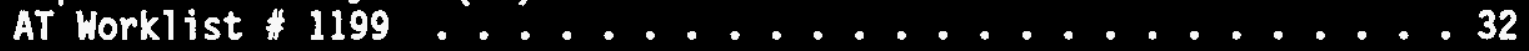

Part II 45-Day Safety Screen Results for Tank 241-B-112, Auger Samples, 95-AUG-014 and 95-AUG-015 .................... 44

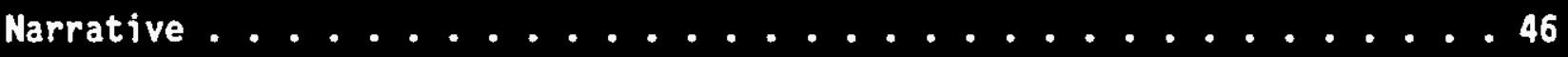

Sample Data Sumary ..................... 50

Undigested Sample Analyses - Direct . . . . . . . . . . 53

Differential Scanning Calorimetry (DSC)

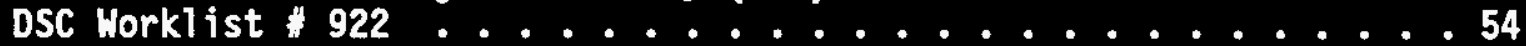

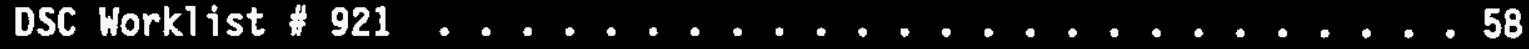


THIS PAGE INTENTIONALLX

LEFT BLANK 


\section{PART I}

MHC-SD-MK-DP-112, REV. 1

REVISED FIIAL REPORT FOR TANK 241-C-203, AUGER SAMPLES 95-AUG-020 AND 95-AUG-021 


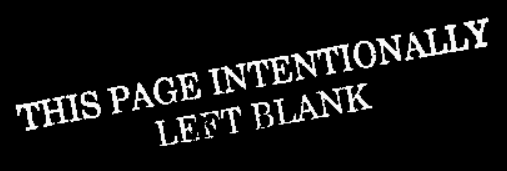


WHC-SD-NH-DP-105, REV. 1

MARRATIVE 


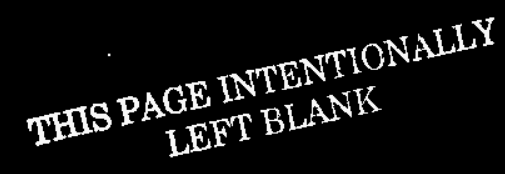


WHC-SD-MH-DP-105, REV. 1

FINAL REPORT FOR TANK 241-B-112, AUGER SAMPLES 95-AUG-014 AND 95-AUG-015

\section{ANALYTICAL SUMMARY}

Two auger samples from tank 241-B-112 (B-112) were received in the 222-S Laboratories and underwent safety screening analyses, consisting of differential scanning calorimetry (DSC), thermogravimetric analysis (TGA), and total alpha activity. All results for all analyses (DSC, TGA, and total alpha) were within the safety screening notification limits specified in the Tank Characterization Plan (TCP) [1]. No notifications nor secondary analyses were required.

SCOPE

This document constitutes the final laboratory report for the tank B-112 auger samples collected on March 16, 1995 (samples 95-AUG-014 and 95-AUG-015). As no additional analyses have been performed, the 45-day report, included as part II of this document (beginning on page 44), contains the data summary tables, discussions of the analytical methods and data, and raw data scans for DSC and TGA analyses. The data summary tables from the 45-day report (pp. 5052) will serve as the final data summary tables as well.

Additional information provided in this revision includes the sample chain-ofcustody sheets, photographs of the extruded auger samples, copies of the laboratory hot-cell notebook pages, and the raw data for the total alpha analyses.

\section{REFERENCE}

[1] R. D. Schreiber, Tank 241-B-112 Tank Characterization Plan, WHC-SD-WMTP-302, Rev. 0, February 6, 1995. 


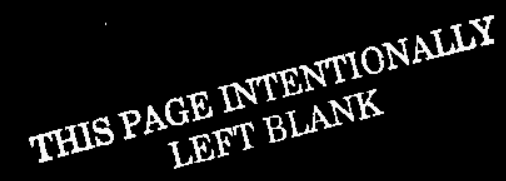


WHC-SD-WM-DP-105, REV. I

\section{CHAIN OF CUSTODY FORMS}




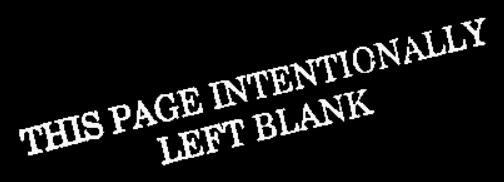




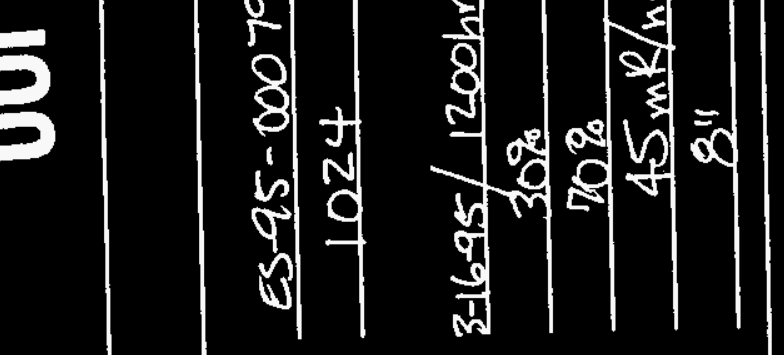

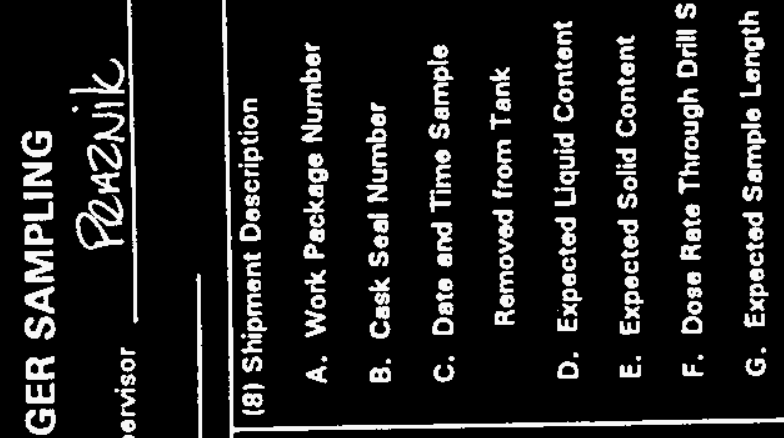

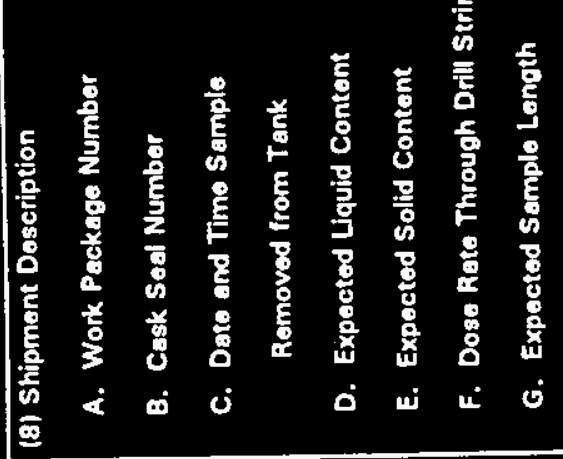

2 实

$\therefore$ क

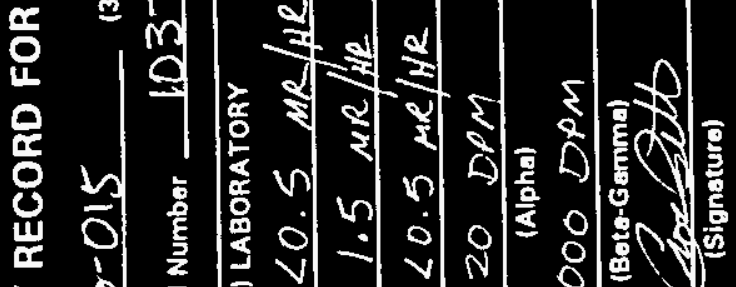

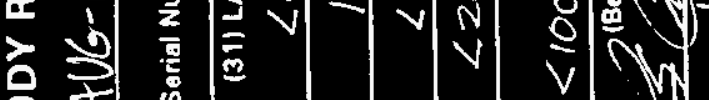

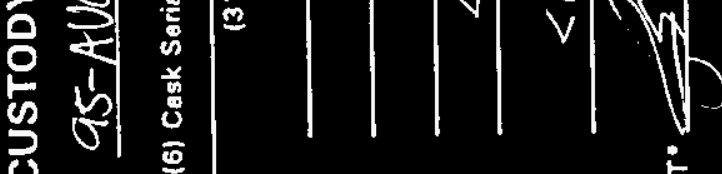

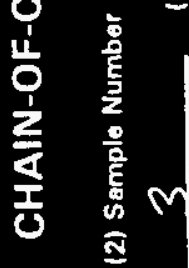

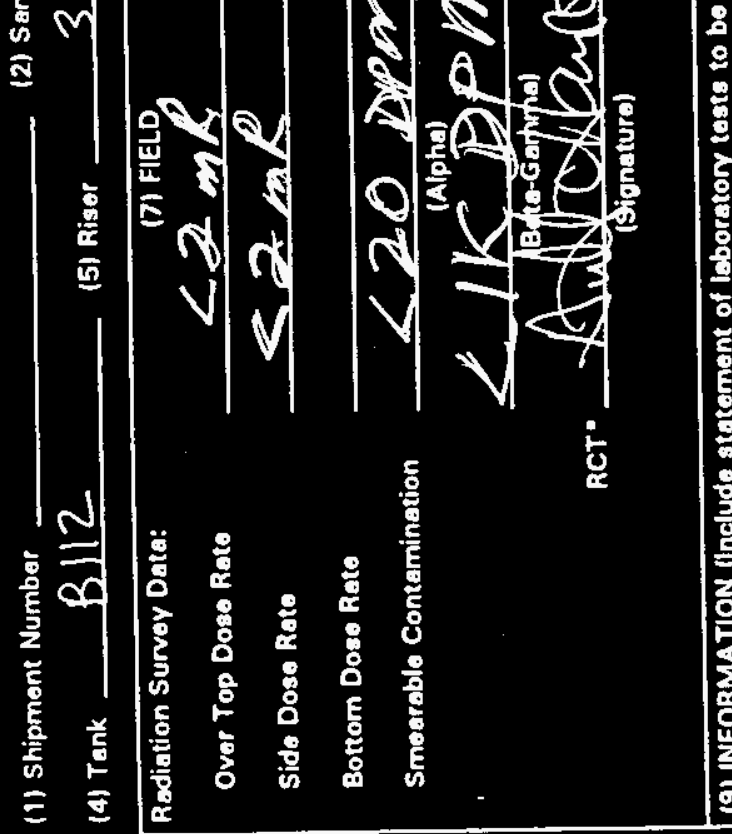

WHC-SD-WM-DP. 105, REV.

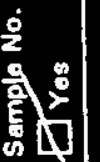
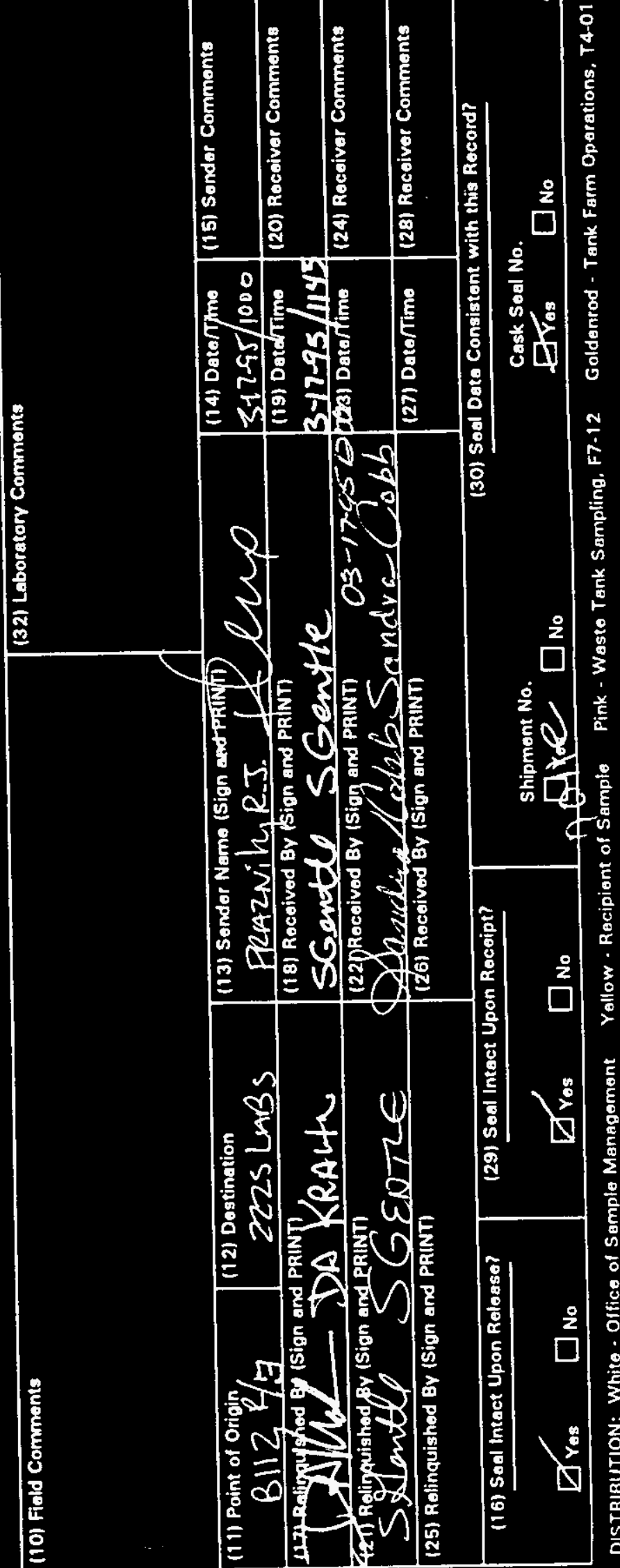
WHC-SD-WM-DP-105, REV. I

HOT CELL LOG BOOK 


$$
\begin{aligned}
& \text { THIS PAGE INTENTIONATI } \\
& \text { IOTIT BLANT }
\end{aligned}
$$


WHC-SD-WM-DP- 105, REV.1

\section{NOTEBOOK/LOGBOOK UNCLASSIFIED COVERSHEET}

\begin{tabular}{|c|c|}
\hline \multirow{3}{*}{$\begin{array}{l}\text { SECTION } \\
\text { RECORD COPY } \\
\text { NOT FOR NIDCIILATIOH } \\
\text { FEB } 171995\end{array}$} & Notabook No. $\mathrm{W} / \mathrm{HC}-\mathrm{N}-11231$ \\
\hline & Date of issue $2-17-95 \quad$ Copy \\
\hline & Title $241-B-112$ \\
\hline \multicolumn{2}{|l|}{ TECEIVED WHC BCSF } \\
\hline Author R. Fuller & $\begin{array}{l}\text { If continued from another notebook } \\
\text { give the notebook number }\end{array}$ \\
\hline
\end{tabular}

This is a Controlled Notebook. The aesigned custodian is responaibla for this book. When the book is comploted, contect your Recorde Management Specialist for a Retention Schedule. Complete Soction II of this form and return the Notebook to Unclassified Document Control, A4-18.

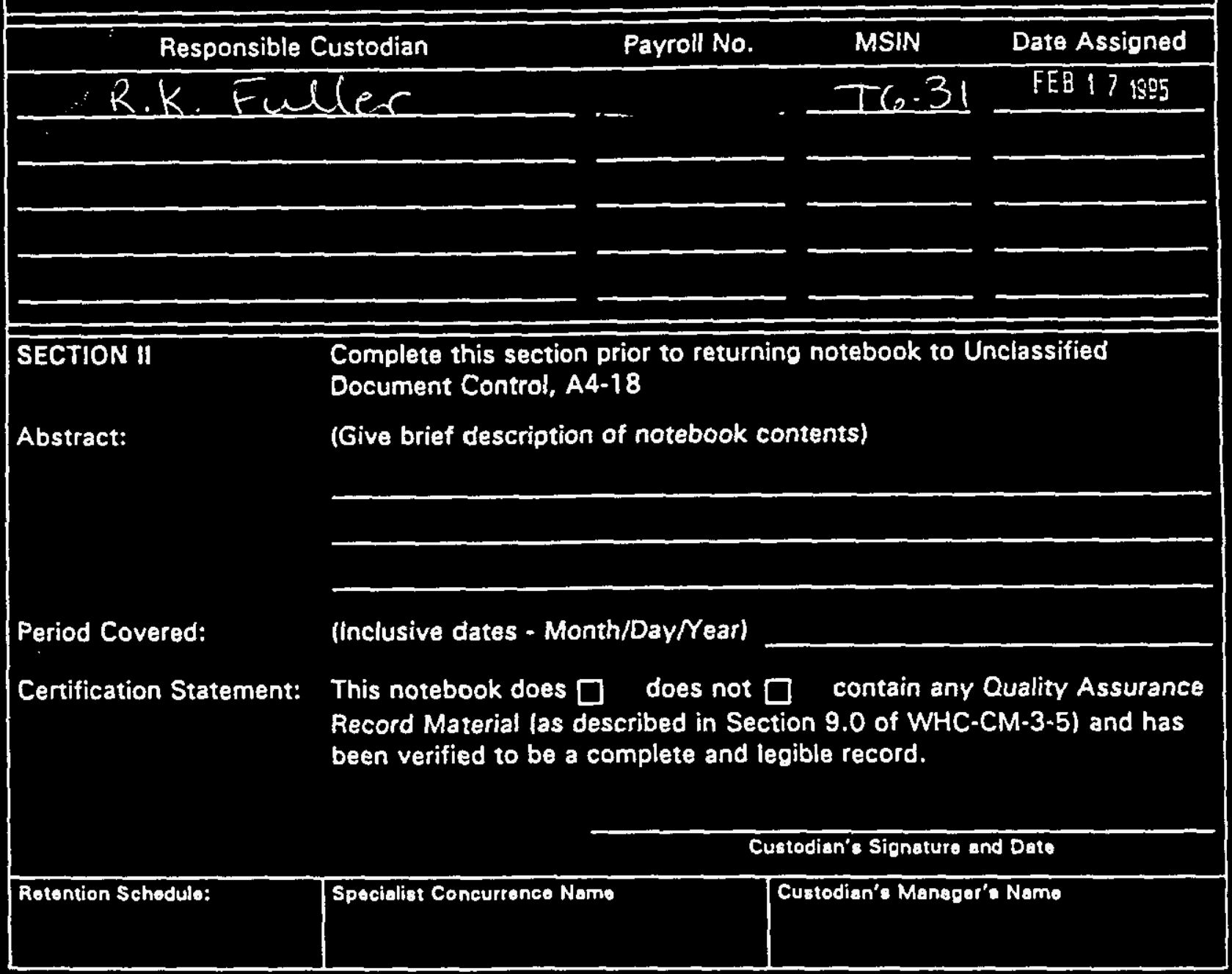




\section{WHC-SO-WM-DP-105, REV. I \\ Table of Contente}

(1) B112 95-AUG-014 Res *7

pageo

(2) B1/2 95-A4G-015 Rise *3

$10-13$ 
WHC-SD-WM-DP-105, REV -1

Pages 2 through 5 of Hot Cell Log Book number WhC-N-1123 Copy 1 were intentionally left Blank. 
Tinpy

CHAIN-OF-CUSTODY RECORD FOR AUGER SAMPLING

(1) Shipment Number (2) Sample Number 95-AVG-Ol4

(3) Supervisor Penznik

(4) Tank B/12 (5) Riser 7

(6) Cask Sorial Numbor 1033

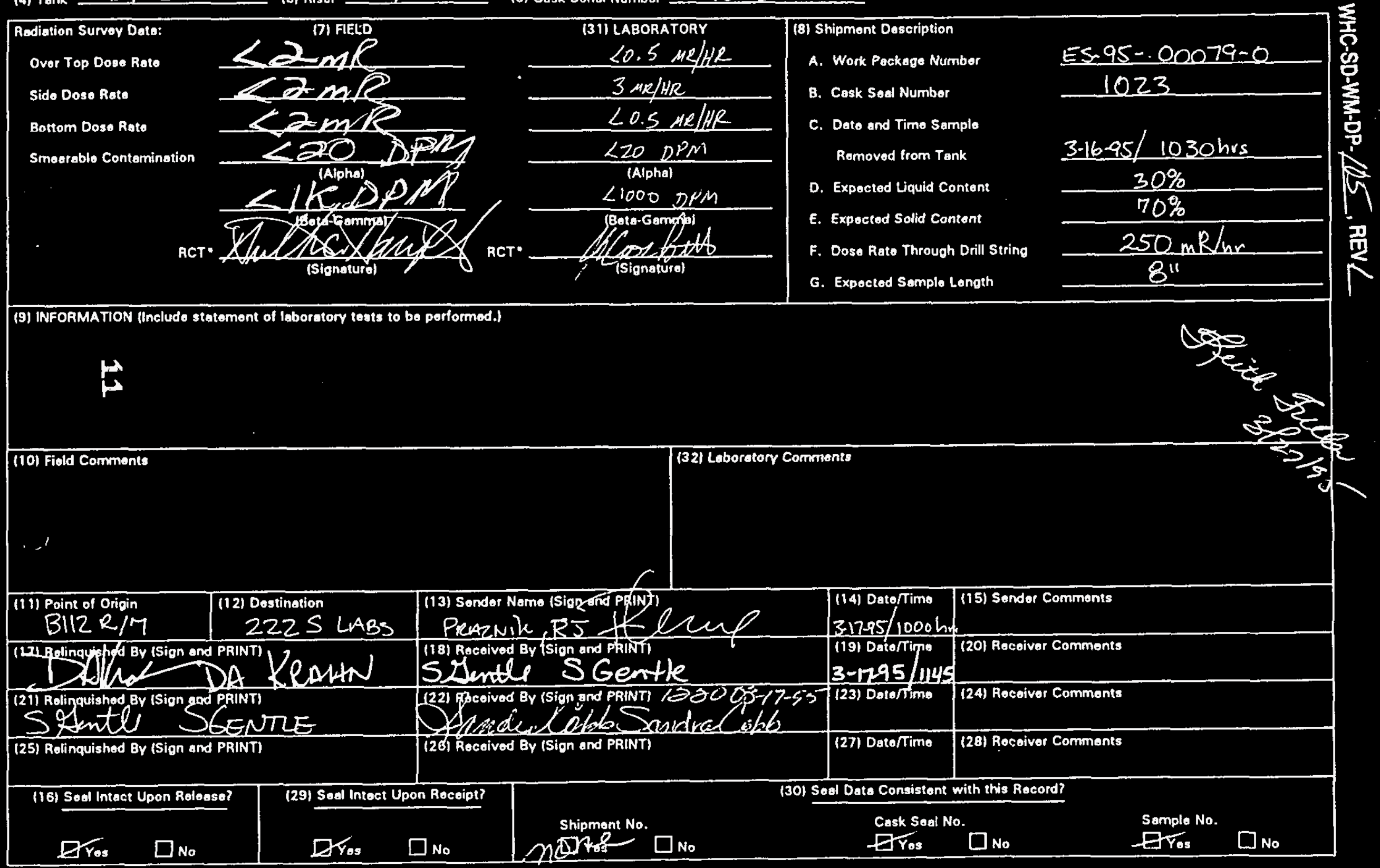

OISTRIBUTION: Whito - Olfice of Samplo Management Yollow - Rocipient of Sample Pink - Wasto Tenk Sampling, F7-12 Goldonrod - Tank Farm Operations, T4-01

BC. $6001-326(03 / 94)$ 
WHC-SO-WM-DP-105, REV. 1

75-ALG-0L4 RISER H 7 3/27/95

Kample \#. 95-A46-014

Cask * : 1033

Dose rate through drile etrixg $=250 \mathrm{mR} / \mathrm{hr}$

Worklist $\# 217$. Takcore nuember $=595 T 000356$

Tape \#6 B Farm

$20 \mathrm{gms}$ wt $=19.99 \mathrm{grams} .500 \mathrm{gm}$ wt $=499.97 \mathrm{grams}$ Temp $=80.6^{\circ} \mathrm{F} \quad$ Elumidity $=20 \%$

Solie:

Total amount of solie matevial recovered was about 7 grams. A emule amont of dask brow solid sample was lecated on plestes *2 threygh *8. Sample

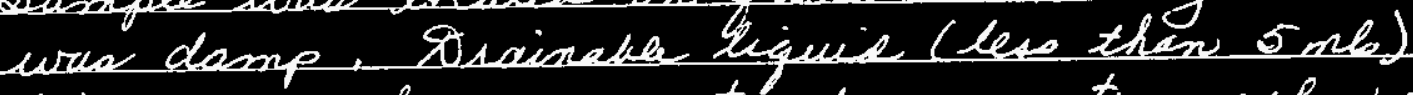
drippes of the anger ento the angen tray when the asger sleve was hemoned. Did nat redower the drainable lequid. Some gray solid yere cellecte from fluter $\# 2$ and $\# 3$ (posibe sruat). Subarmpled the remaiaing flutwo (\#4 through $\# 8$ ).

Sulsample trpasmatio:

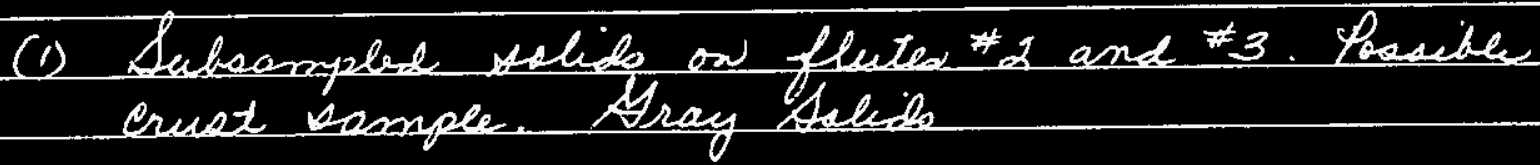

- Vial *6499 (20 ml-vial sige)

-2.02 gramo recoured

(2) Subsampled solid on ylutw \#4 thrngh *8.

Remaining valide. Browo selids.

- Vial * 6633 (20 ml - wall sije)

- 5.70 grame recoverud 


\section{WHC-SD-WM-DP-105, REV 1}

$3 k 2 / 45$

- tid not divide vamper into 1/2 seginenta deu to the lack of materil.

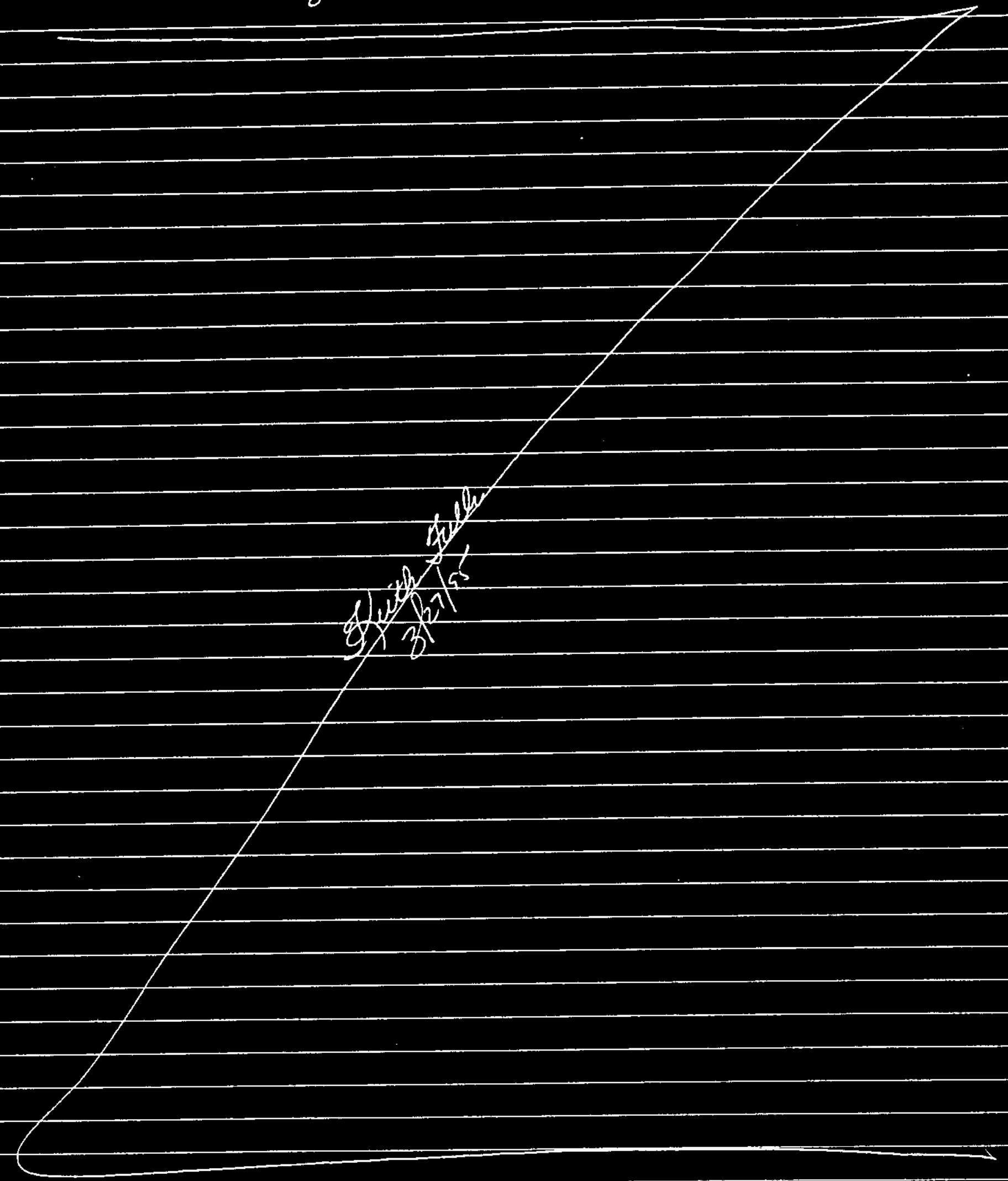




$$
\begin{gathered}
\text { WHC-SD-WM-DP-105, REV.1 } \\
\text { B112 95-AUCi-C14 Rise, } 47 \\
\text { Photograph }
\end{gathered}
$$
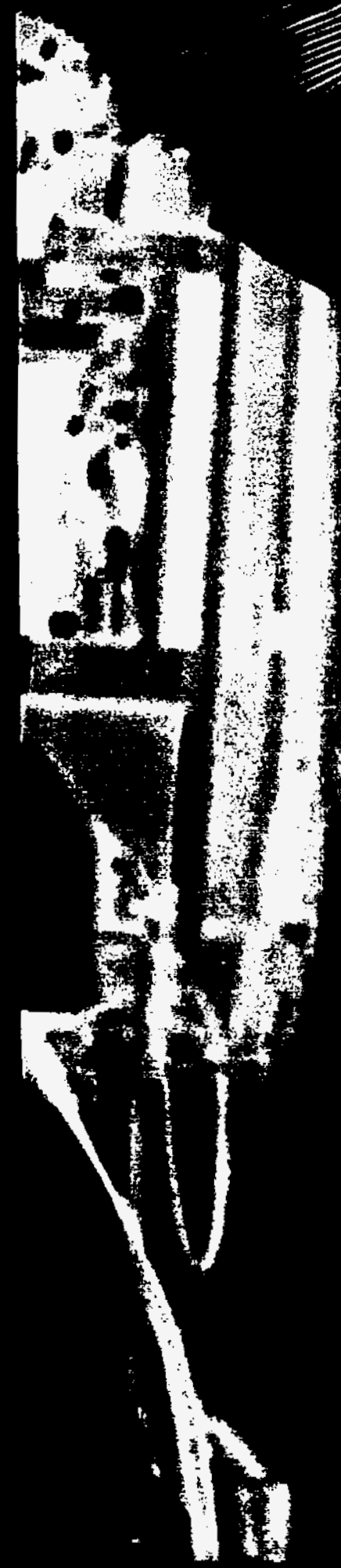
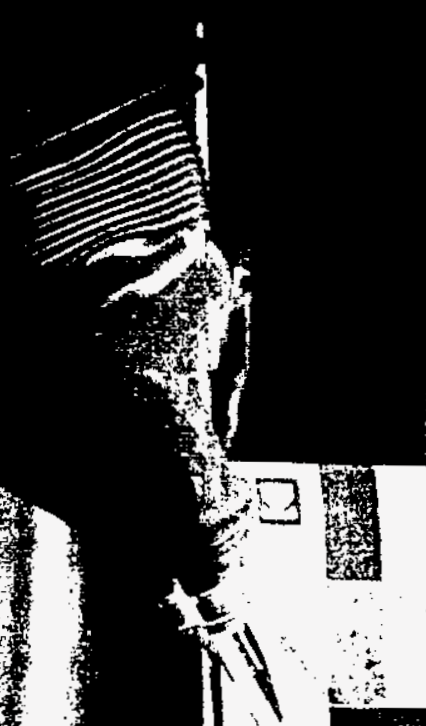

,
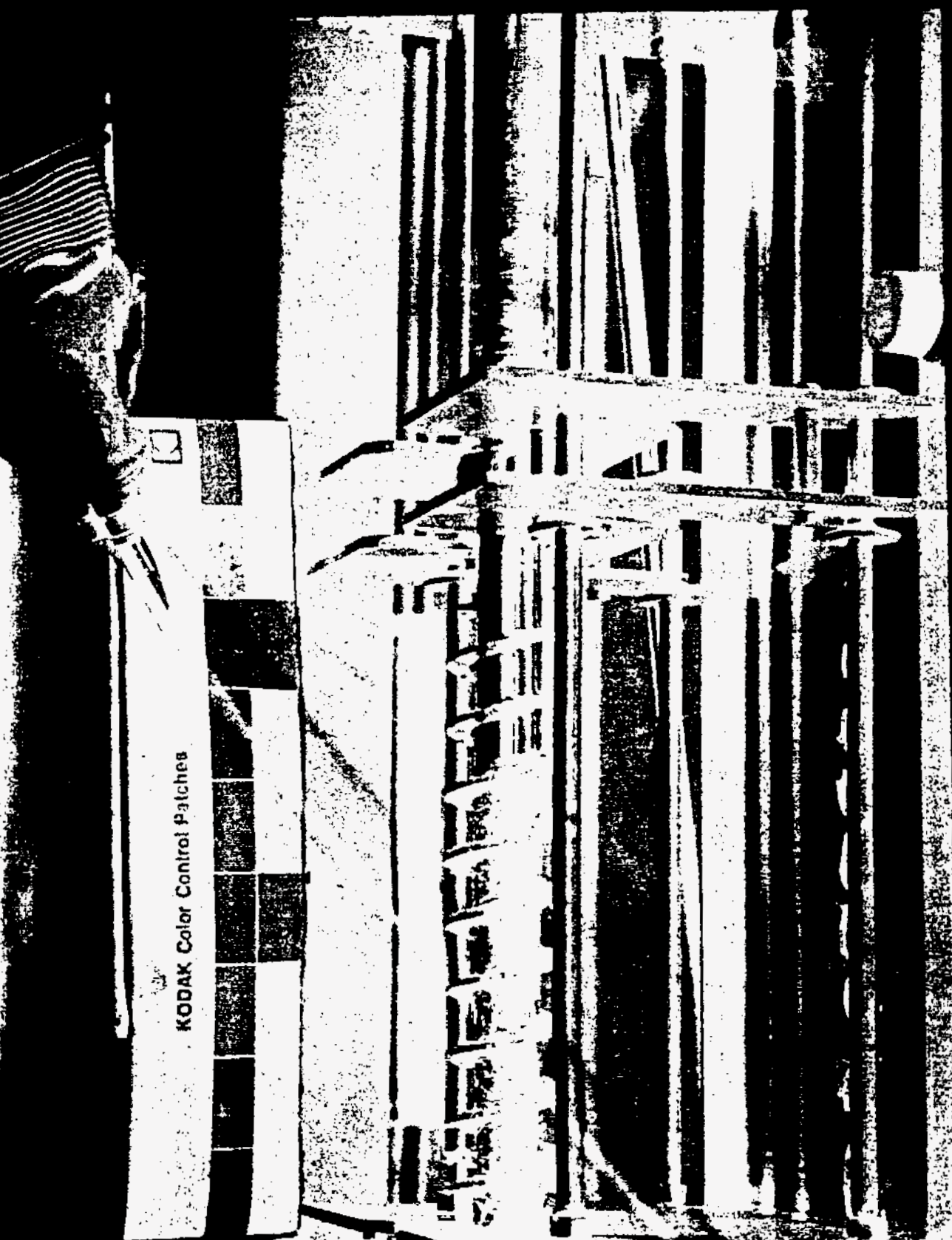

il al

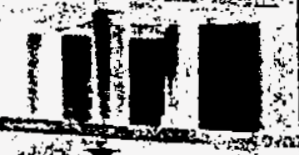
$p$

$\frac{-2}{|\pi|} \mid$
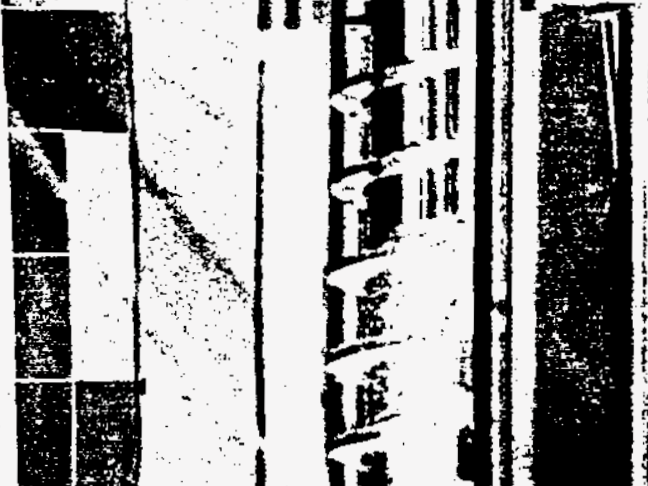

18.

(1)

L

tis

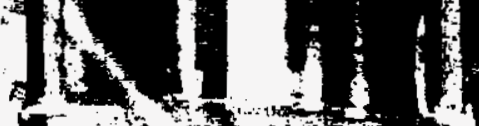

ing

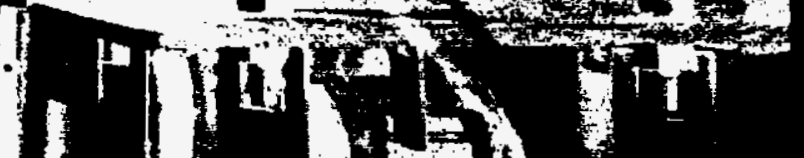

ind

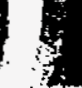

$\frac{1}{x+2}$

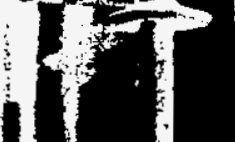

1

.)

1,

4

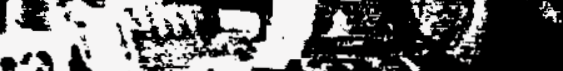

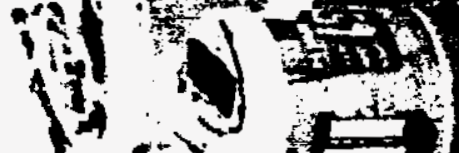

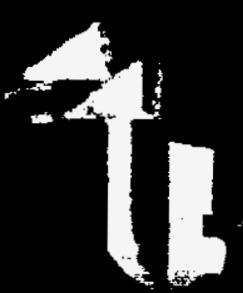

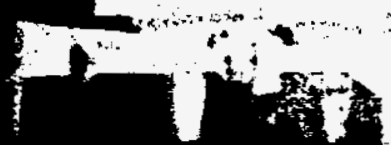

$i^{-1} \cdot x^{2}$

$+1$ 
WHC-SD-WM-DP- 105, REV $\perp$

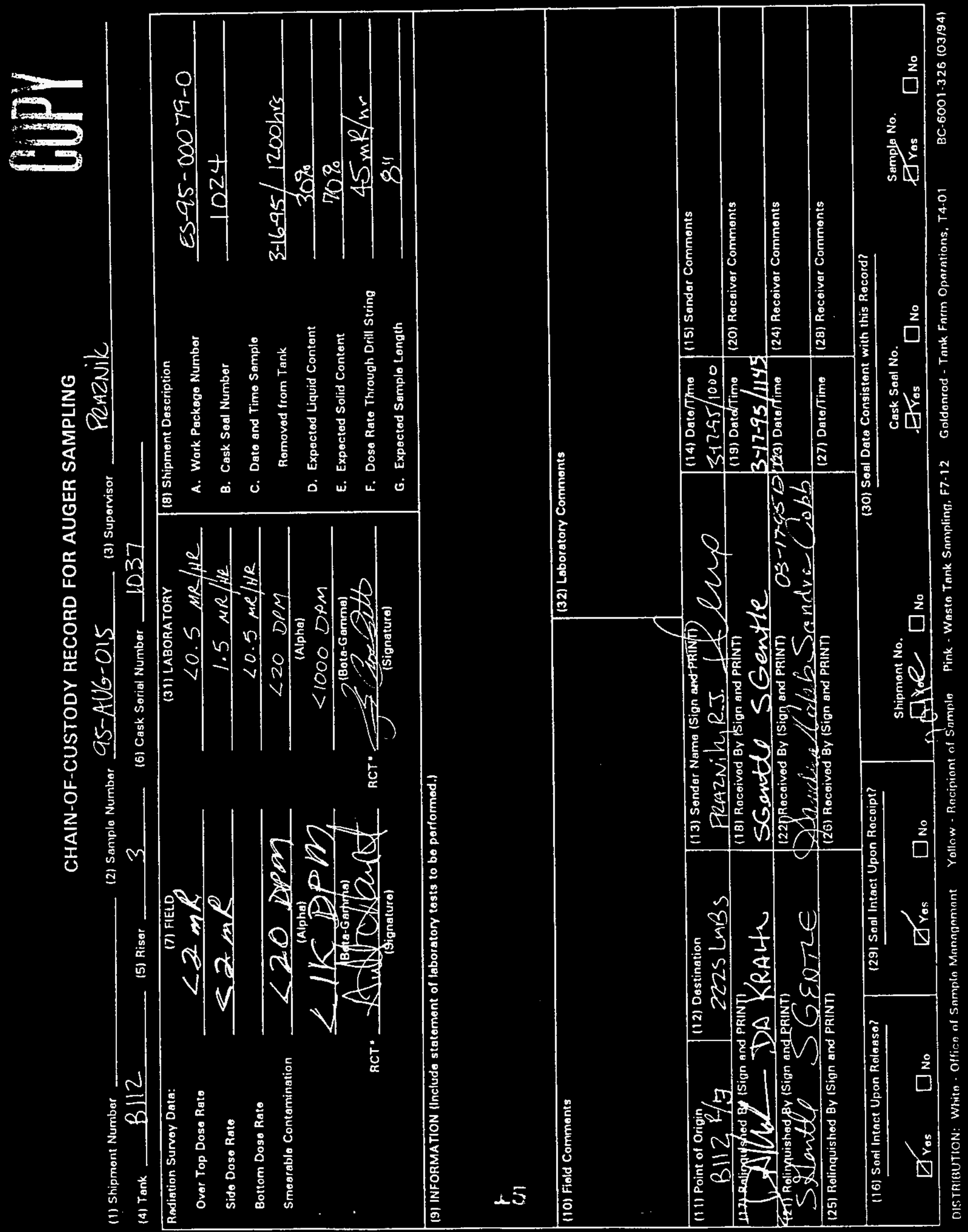


$3 / 28 / 55$

$$
b i
$$

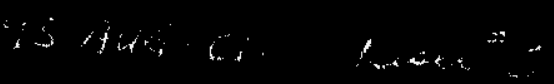

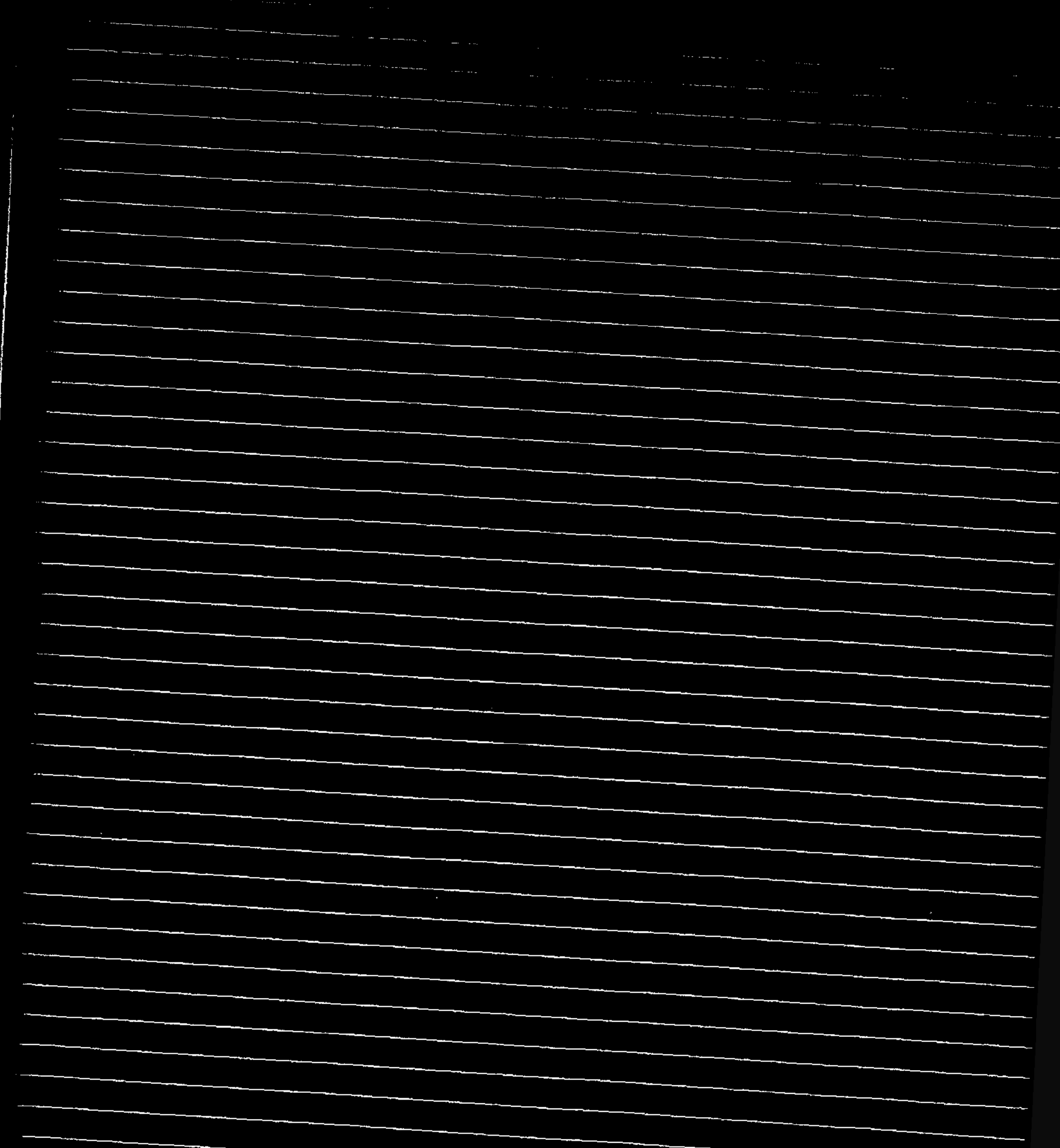




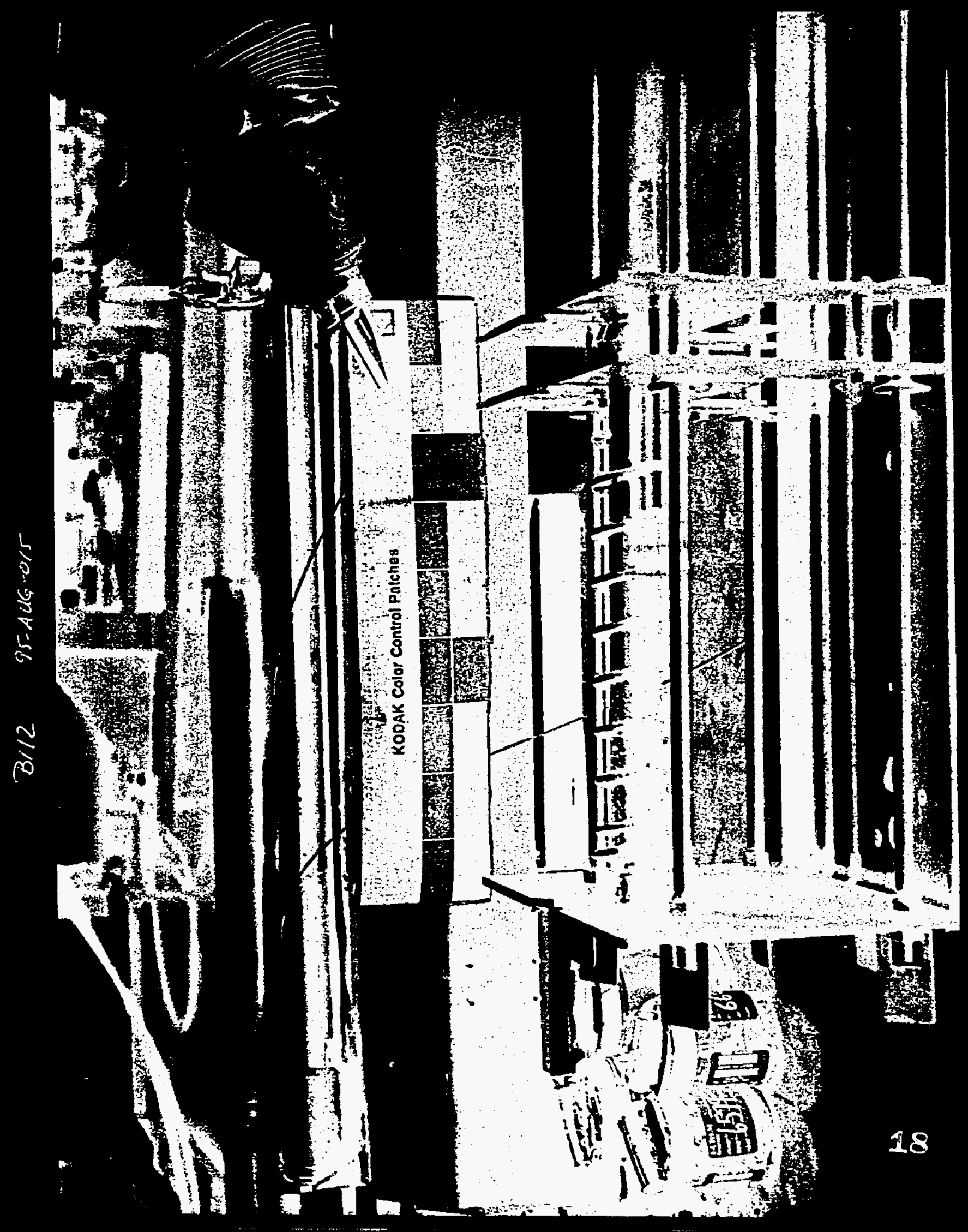




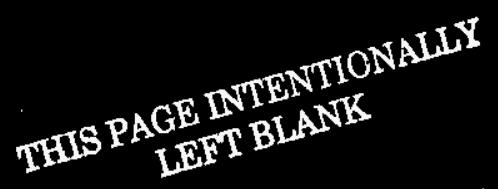


WHC-SD-MH-DP-105, REV. 1

PHOTOGRAPHS 


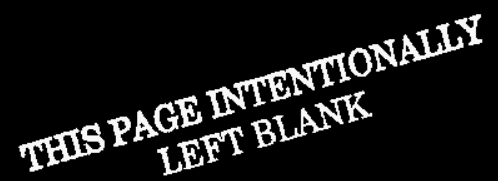


WHC-SD-WM-DP. 105, REV. I

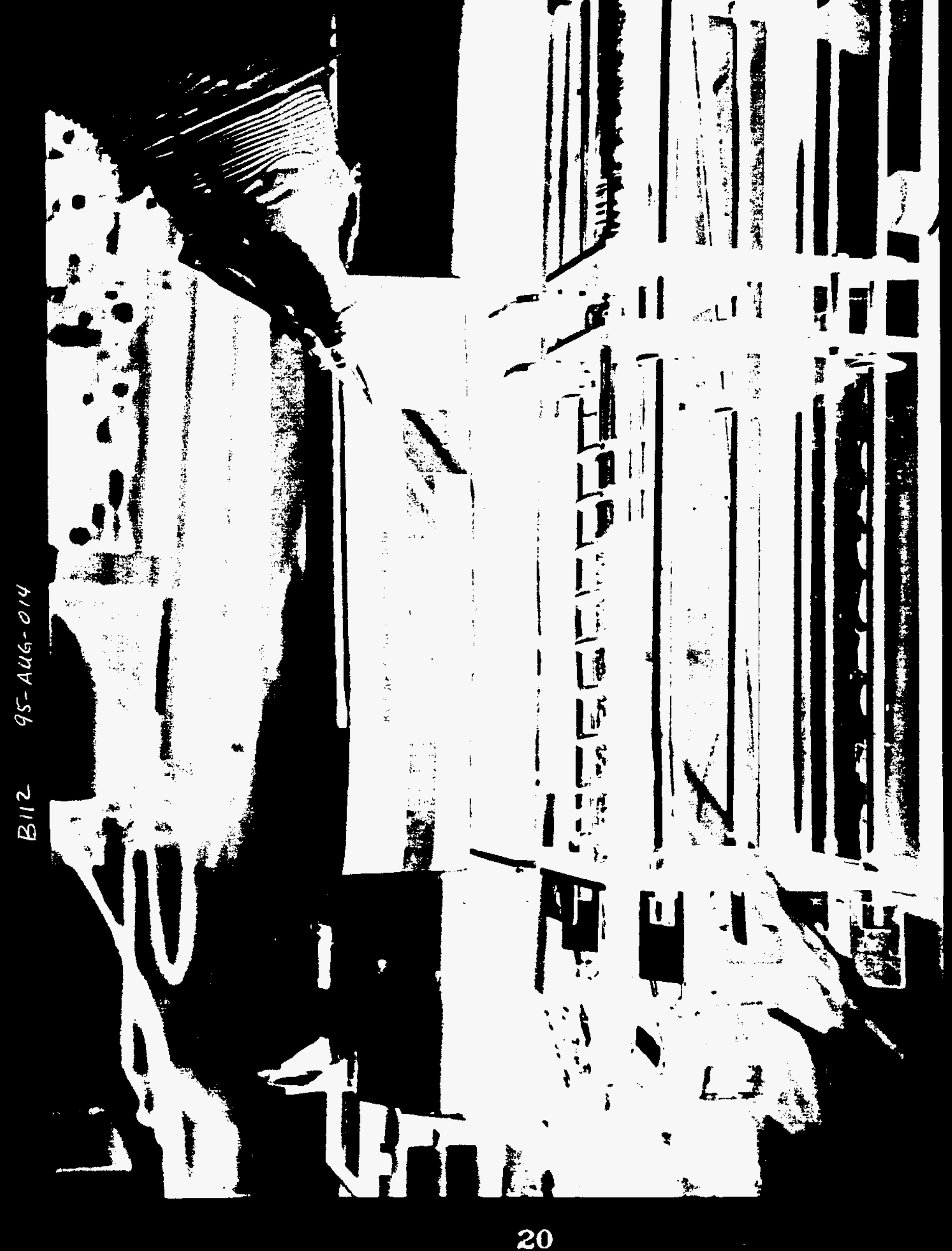


WHC-SD-WM-DP. 105 , REV. 1

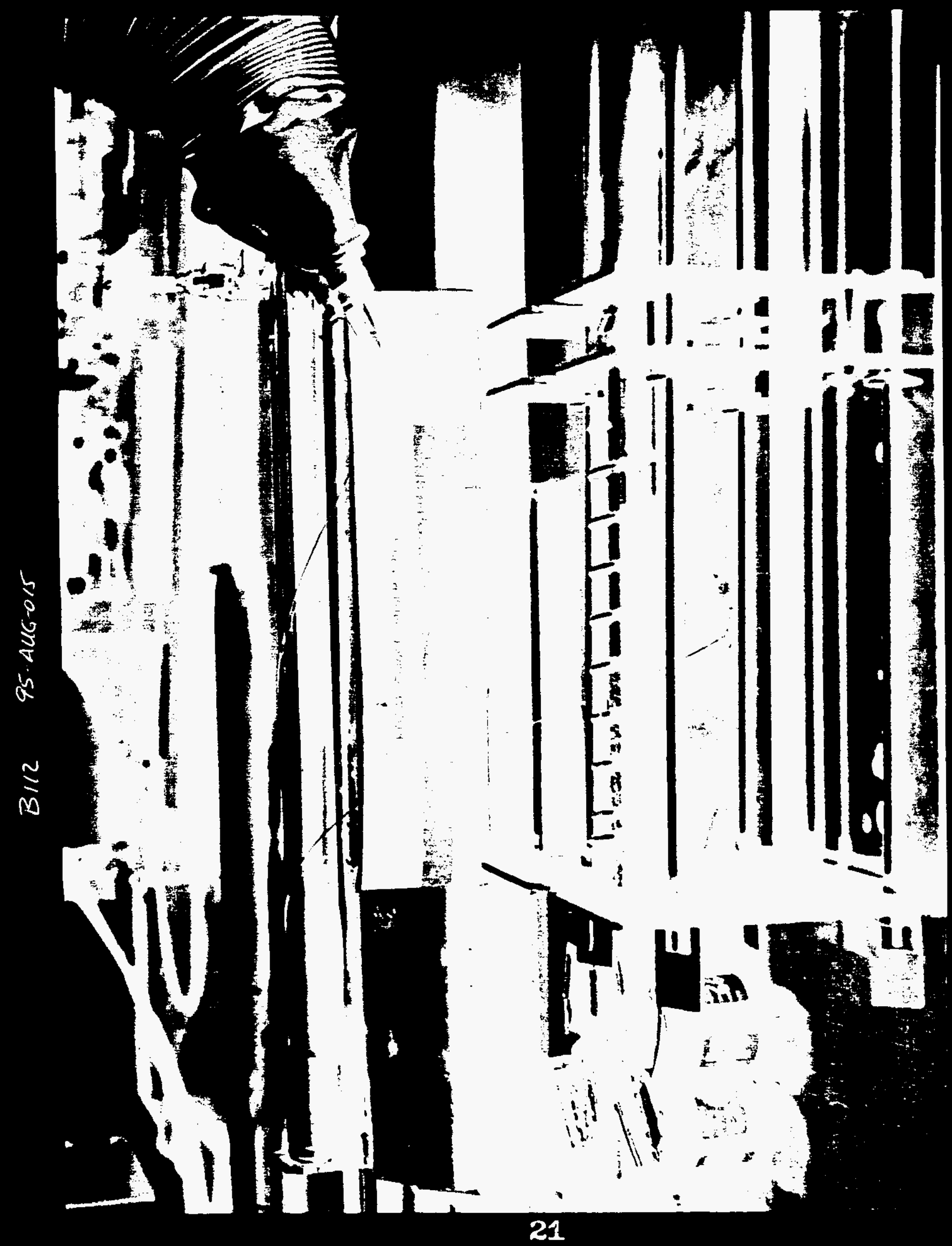




$$
\begin{aligned}
& \text { THIS PAGE INTENTIONAIIT } \\
& \text { LEFT BLANK }
\end{aligned}
$$


WHC-SD-WH-DP-105, REV. 1

SAMPLE HANDLING

22 


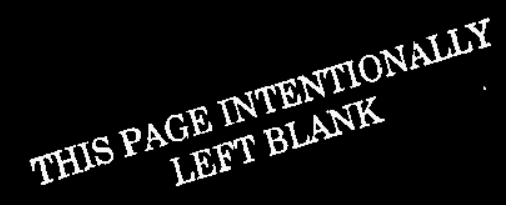




\section{LABCORE Data Entry Template for Worklist\# 862}

Analyst: $\quad C C$ Instrument: BA000_NA Book \# NA

Method: LO-160-103 Rev/Mod A-7

Worklist Comment: B-112 95-AUG-015 Riser 3 Extrusion

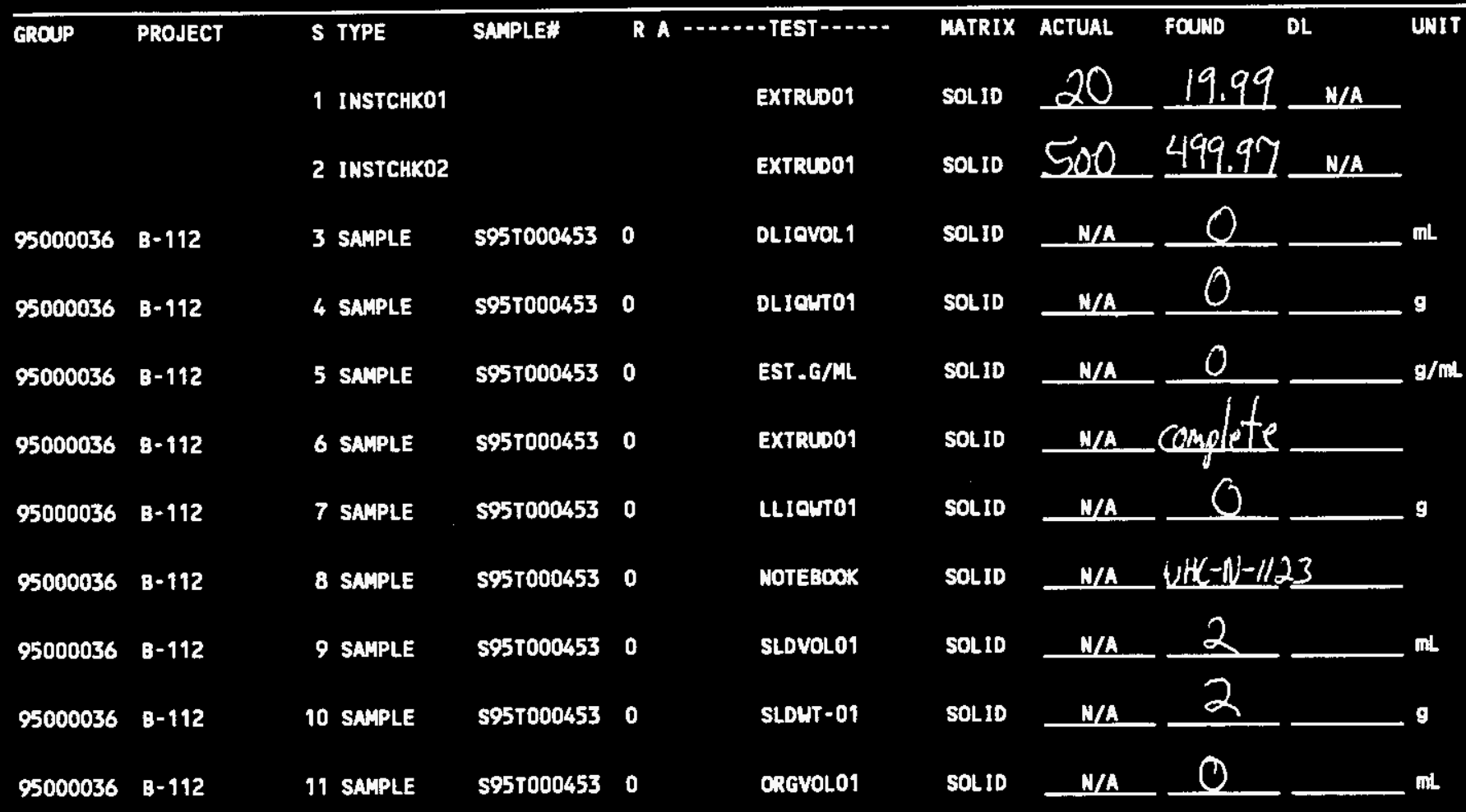

Final page for worklist \# 862

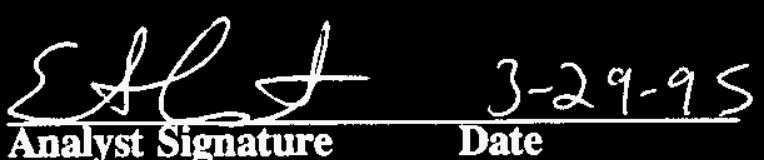

Analyst Stgnature Date

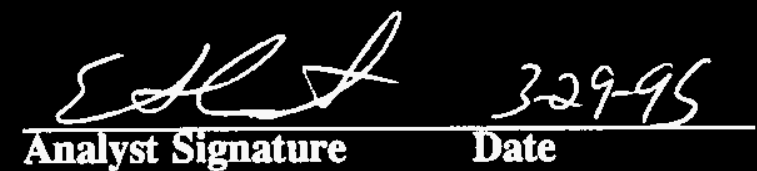

Was origimally worklist 4778 .

Data Entry Comments:

Units shown for $Q C$ (SPK \& STD) may not reflect the actual units. $D L=$ Detection Limit, $S=$ Worklist Slot Number, $R=$ Replicate Number, $A=$ Aliquot Code. 


\section{LABCORE Data Entry Template for Worklist\# 777}

Analyst: $\quad$ RK7 Instrument: BA000

Book \#

Method: LO-160-103 Rev/Mod A-7

Worklist Comment: B-112 95-AUG-014 Riser \#7

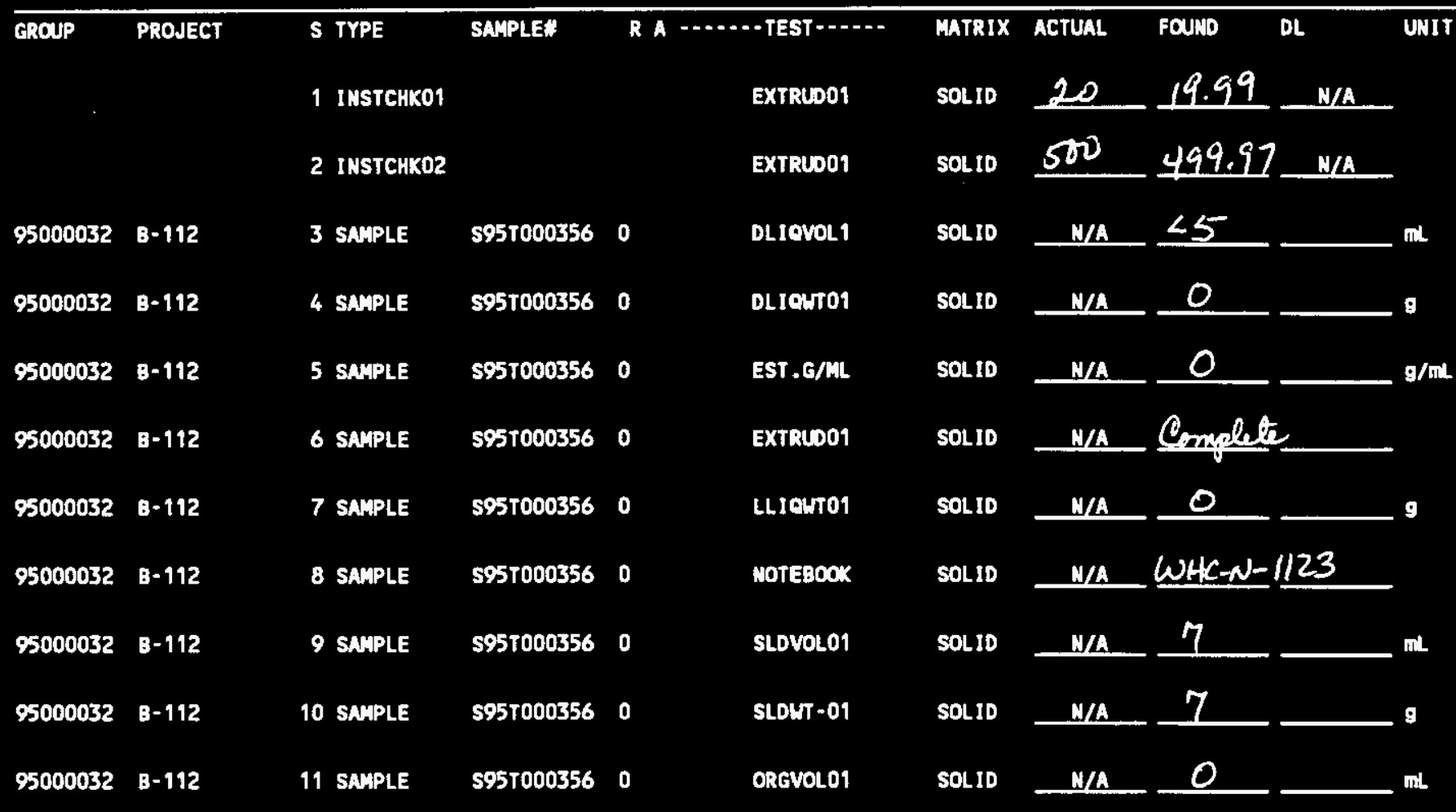

Final page for worklist \# 777

Heith fule 3/28/95

Andyst Signature Date

Analyst Signature Date

Data Entry Comments:

Units shown for $Q C$ (SPK \& STD) may not reflect the actual units. $D L=$ Detection Limit, $S=$ Worklist Slot Number, $R=$ Replicate Number, $A=$ Aliquot Code. 
WHC-SD-WM-DP-105, REY. 1

SAMPLE PREPARATIONS

25 


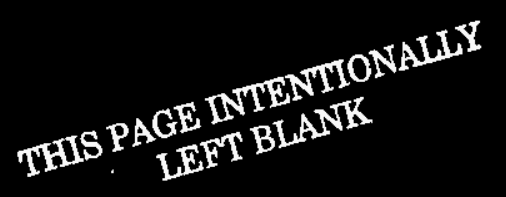




\section{LABCORE Data Entry Template for Worklist\# 1192}

Page: $\quad l$

\section{Analyst:

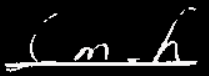 \\ Instrument: FUSO1 $\mathrm{A} / \mathrm{LL} 6 \mathrm{C}$ \\ Book \#}

Method: LA-549-141 Rev/Mod C. 2

Worklist Comment: SUPER RUSH B-112 FUSION - 357->864, 454-> 865

\begin{tabular}{|c|c|c|c|c|c|c|c|}
\hline \multirow[t]{2}{*}{ GROUP } & \multirow[t]{2}{*}{ PROJECT } & $S$ TYPE & SAMPLE\# & \multicolumn{2}{|c|}{ R A $\cdots$ - -TEST - . . } & MATRIX & ACTUAL \\
\hline & & 1 BLNK-P & & & FUSION01 & SOLID & 25 Cine 2 \\
\hline 95000032 & B-112 & $\begin{array}{l}2 \text { SAMPLE } \\
.51549\end{array}$ & $\begin{array}{r}595 T 000864 \\
25 \mathrm{C} \text {. }\end{array}$ & OF & FUSIONO1 & SOLID & 2,0616 \\
\hline 95000032 & B-112 & $\begin{array}{c}3 \text { oup } \\
.51979\end{array}$ & $\begin{array}{l}5951000864 \\
250 \mathrm{mC}\end{array}$ & $0 \mathrm{~F}$ & FUSIONO1 & SOLID & 2.06162 .0785 \\
\hline 95000036 & B-112 & $\begin{array}{l}4 \text { SAMPLE } \\
399-25\end{array}$ & 250 in & OF & FUSI OW01 & SOLID & 10968 \\
\hline 95000036 & B-112 & $\begin{array}{l}5 \text { Dup } \\
45079\end{array}$ & $\begin{array}{l}5957000865 \\
25 C^{\circ} m\end{array}$ & $8^{\circ}$ & FUSI ON01 & SOLID & 1.096819920 \\
\hline
\end{tabular}

\section{Final page for worklist \# 1192}
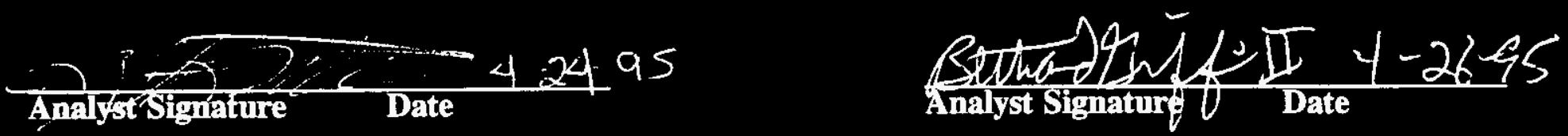

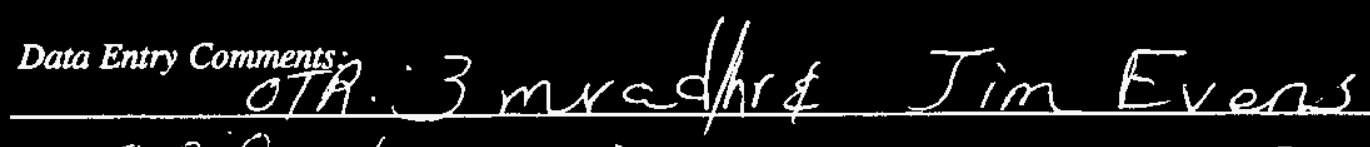

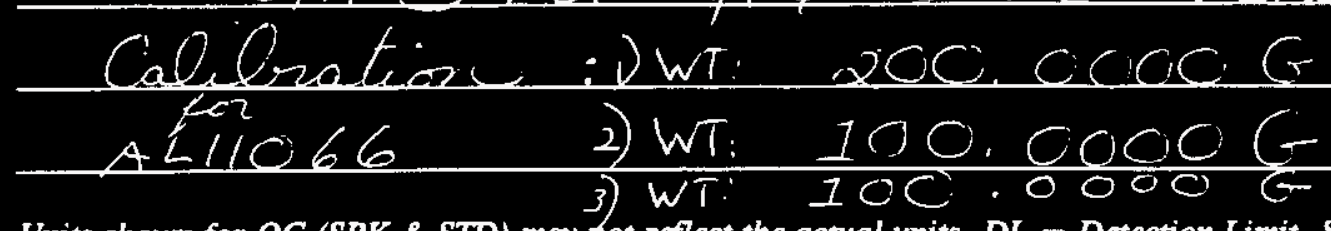

Units shown for $Q C$ (SPK \& STD) masyot reflect the actual units. $D L=$ Detection Limit, $S=$ Worklist Slot Number,

$R=$ Replicate Number, $A=$ Aliquot Code. 
WHC-SD-WH-DP-105, REV. I

INORGANIC AMALYSES 


\section{THIS PAGE INTENTIONALLY}

LEFT BLANK 


\section{LABCORE Data Entry Template for Worklist\# 1207}

Analyst: BDV Instrument: DSC01

Book \# -

Method: LA-514-113 Rev/Mod

$-$

Worklist Comment: Calculated dry DSC for B-112. bdv

WHC-SD-WM-DP- 105, REV. I

\begin{tabular}{|c|c|c|c|c|c|c|c|c|c|c|}
\hline GROUP & PROJECT & S TYPE & SAMPLE* & & - TEST- - . - & MATRIX & ACTUAL & FOUND & DL & UNIT \\
\hline 95000032 & B-112 & 1 SAMPLE & S95T000357 & 0 & DSC-02 & SOLID & N/A & 31.9 & & Joules/g Dry \\
\hline 95000032 & $8-112$ & 2 DUP & S95T000357 & 0 & DSC-02 & SOLID & 31.9 & 8.3 & $\mathrm{~N} / \mathrm{A}$ & Joules/g Dry \\
\hline 95000032 & B-112 & 3 DUP2 & \$95T000357 & 0 & DSC-02 & SOLID & 31.9 & 27.2 & N/A & Joules/g Dry \\
\hline 95000032 & B-112 & 4 SAMPLE & $595 T 000452$ & 0 & DSC-02 & SOLID & N/A & $\varnothing$ & & Joules/g Dry \\
\hline 95000032 & B-112 & 5 OUP & 595T000452 & 0 & DSC -02 & SOLID & $\varnothing$ & $\mathscr{Q}$ & N/A & Joules/g Dry \\
\hline 95000036 & $B-112$ & 6 SAMPLE & S95T000454 & 0 & DSC-02 & SOLID & H/A & $\phi$ & & Joules/g Dry \\
\hline 95000036 & $8-112$ & 7 DuP & S95T000454 & 0 & DSC-02 & SOLID & $\varnothing$ & $\phi$ & N/A & Joules/g Dry \\
\hline
\end{tabular}

\section{Final page for worklist \# 1207}

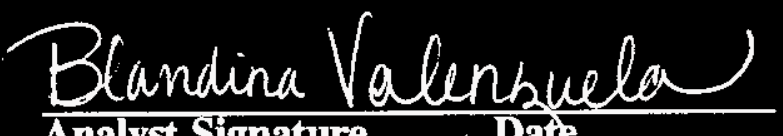

Analyst Signature 


\begin{tabular}{|c|c|c|c|}
\hline & $\begin{array}{l}\text { WHC } \\
\text { WHC }\end{array}$ & $\begin{array}{l}\text { D-WM-DP- } 105 \text {, REV }-1 \\
\text { D-WM-DP-105, REV. } 0\end{array}$ & \\
\hline & CALCU & TED DRY DSC & \\
\hline SAMPLE NO. & DSC RESULT (J/g) & TEA RESULT (\% water) & DRY DSC RESULT \\
\hline 5957000357 & 21.9 & 31.34 & 31.9 \\
\hline $357 D$ & 5.7 & 31.34 & 8.3 \\
\hline 35702 & 18.7 & 31.34 & 27.2 \\
\hline 452 & $\varnothing$ & 43.42 & $\varnothing$ \\
\hline 452D & $\emptyset$ & 43.42 & $\varnothing$ \\
\hline 454 & $\varnothing$ & 46.36 & $\phi$ \\
\hline 4540 & $\varnothing$ & 46.36 & 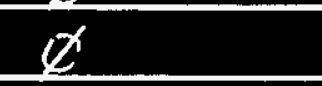 \\
\hline & & & \\
\hline & & & \\
\hline & & & \\
\hline & & & \\
\hline & & & \\
\hline & & & \\
\hline & & & \\
\hline & $\cdot$ & & \\
\hline & & & \\
\hline & & & \\
\hline & & & \\
\hline & & & \\
\hline & & & \\
\hline & & & \\
\hline & & & \\
\hline
\end{tabular}


WHC-SD-WM-DP-105, REV. 1

\section{RADIOCHEMICAL ANALYSES}

24 


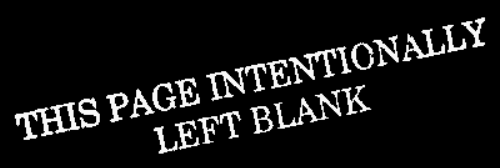




\section{LABCORE Data Entry Template for Worklist\# 1199}

\section{Analyst:

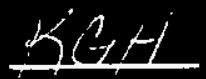 \\ Instrument: $\mathrm{ABO0} \$ / 5$ \\ Book \# $11.7123=2$}

Method: LA-508-101 Rev/Mod D-2

LiB 26872

Worklist Comment: Determine sample size using Ludlum. SLF WHC-SD-WM-DP. 105 , REV.L

\begin{tabular}{|c|c|c|c|c|c|c|c|c|c|c|}
\hline \multirow[t]{6}{*}{ GROUP } & PROJECT & S TYPE & SAMPLE: & R A & -..... TEST $\cdots$ & MATRIX & ACTUAL & FOUND & DL & UNIT \\
\hline & & 1 STO & & & CALPHAO1 ALPHAO1 & SOLID & & & N/A & $\mathrm{uCi} / \mathrm{g}$ \\
\hline & & 1 STD & & & DALPAO1 ALPHAO1E & SOL.10 & & & N/A & X ct. Error \\
\hline & & 2 BLNK-PREP & & & ALPHAO1 ALPHAO1 & SOLID & & & $N / A$ & $\mathrm{uCi} / \mathrm{g}$ \\
\hline & & 2 BLNK-PREP & & & ALPHAO1 ALPHAO1E & SOLID & & & $N / A$ & X Ct. Error \\
\hline & & 3 BLNK/BKG & 0.45 & & AALPHAO1 ALPHAO1 & SOLIO & & & N/A & uci/g \\
\hline 95000032 & $B \cdot 112$ & 4 SAMPLE & 595T000481 & OF & ALPHAOI ALPHAOT & SOLIO & $\mathbf{N} / \mathbf{A}$ & & & uCi/g \\
\hline 95000032 & $B-112$ & 4 SAMPLE & $595 \mathrm{r000481}$ & O F & DALPHAO1 ALPHAO1E & SOLID & $\mathbf{N} / \mathbf{A}$ & & & X Ct. Error \\
\hline 95000032 & B-112 & 5 DUP & $\$ 957000481$ & OF & ALPHAO1 ALPHAO1 & SOLID & & & $\mathrm{N} / \mathrm{A}$ & uCi/g \\
\hline 95000032 & B-112 & 5 DUP & S95T000481 & $0 F$ & DALPHA01 ALPHAO1E & SOL ID & & & $N / A$ & X Ct. Error \\
\hline \multirow[t]{6}{*}{95000032} & B-112 & 6 SPK & 5957000481 & OF & CALPHAO1 ALPHAO1 & SOLID & . & & $N / A$ & uCi/g \\
\hline & & 7 STO & & & DALPHAO1 ALPHAO1 & SOLID & & & $N / A$ & $\mathrm{uCi} / \mathrm{s}$ \\
\hline & & 7 sto & & & ALPHA01 ALPHAO1E & SOLID & & & $\mathbf{N} / \mathbf{A}$ & X ct. Error \\
\hline & & 8 BLNK-PREP & & & ALPHAO1 ALPHAO1 & SOL ID & & & $\mathrm{N} / \mathrm{A}$ & $\mathrm{uCi} / \mathrm{g}$ \\
\hline & & 8 BLNK-PREP & & & BALPHAOT ALPHAOTE & SOL I0 & & & $\mathbf{N} / \mathbf{A}$ & X Ct. Error \\
\hline & & 9 BLNK/BKG & .24 & & ALPHAO1 ALPHAO1 & SOLID & & & $\mathrm{N} / \mathrm{A}$ & $\mathbf{u C i} / \mathrm{g}$ \\
\hline 95000032 & $8-112$ & 10 SAMPLE & 5957000864 & OF & ALPHA01 ALPHAO1 & SOLID & $N / A$ & & & uci/g \\
\hline 95000032 & B-112 & 10 SAMPLE & $595 T 000864$ & OF & AALPHA01 ALPHA01E & SOLID & $N / A$ & & & x ct. Error \\
\hline 95000032 & $B-112$ & 11 DUP & S95T000864 & O F & DALPHAO1 ALPHAOT & SOLID & & & $\mathrm{N} / \mathrm{A}$ & $\mathrm{uCi} / \mathrm{g}$ \\
\hline
\end{tabular}

Data Entry Comments:

Low SPK resovery is the resmlt of Dish eecosion by Nel.

No refme requented.

A-ph $5 / 1 / 95$

Units shown for QC (SPK \& STD) may not reflect the actual units. $D L=$ Detection Limit, $S=$ Worklist Slot Number, $R=$ Replicate Number, $A=$ Aliquot Code. 


\section{LABCORE Data Entry Template for Worklist\# 1199}

\begin{tabular}{|c|c|c|c|c|c|c|c|c|c|c|}
\hline$\overline{\text { GROUP }}$ & PROJECT & S TYPE & SAMPLE\# & $\overline{R T}$ & $\cdots \cdots$ TEST $\cdots \cdots$ & MATRIX & ACTUAL & FOUND & $\overline{D L}$ & UNIT \\
\hline 95000032 & B-112 & 11 DUP & 5951000864 & OF & QALPHAOI ALPHAO1E & SOLID & & & $N / A$ & X Ct. Error \\
\hline 95000036 & B-112 & 12 SAMPLE & 5957000865 & $\mathrm{OF}$ & ลALPHAOI ALPHAO1 & SOLID & N/A & & & uCi/g \\
\hline 95000036 & 8-112 & 12 SAMPLE & $595 T 000865$ & $\mathrm{OF}$ & QALPHAO1 ALPHAO1E & SOLID & N/A & & & X ct. Error \\
\hline 95000036 & B-112 & 13 DUP & S95T000865 & OF & EALPHAO1 ALPHAO1 & SOL ID & & & $\mathbf{E} / \mathbf{A}$ & $u c i / g$ \\
\hline 95000036 & B-112 & 13 DUP & 5957000865 & $0 \mathrm{~F}$ & CALPHAO1 ALPHAOTE & SOLID & & & $\mathbf{N} / \mathbf{A}$ & X ct. Error \\
\hline
\end{tabular}

\section{Final page for worklist \# 1199}
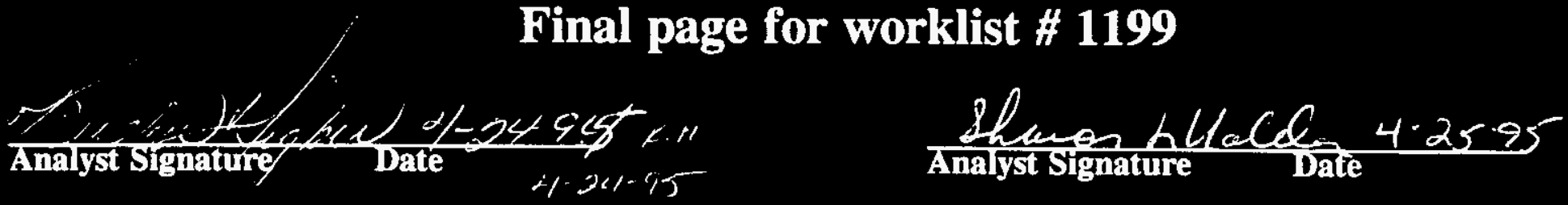

Data Entry Comments:

Units shown for $Q C$ (SPK \& STD) may not reflect the actual units. $D L=$ Detection Limit, $S=$ Worklist Slot Number, $R=$ Replicate Number, $A=$ Aliquot Code. 


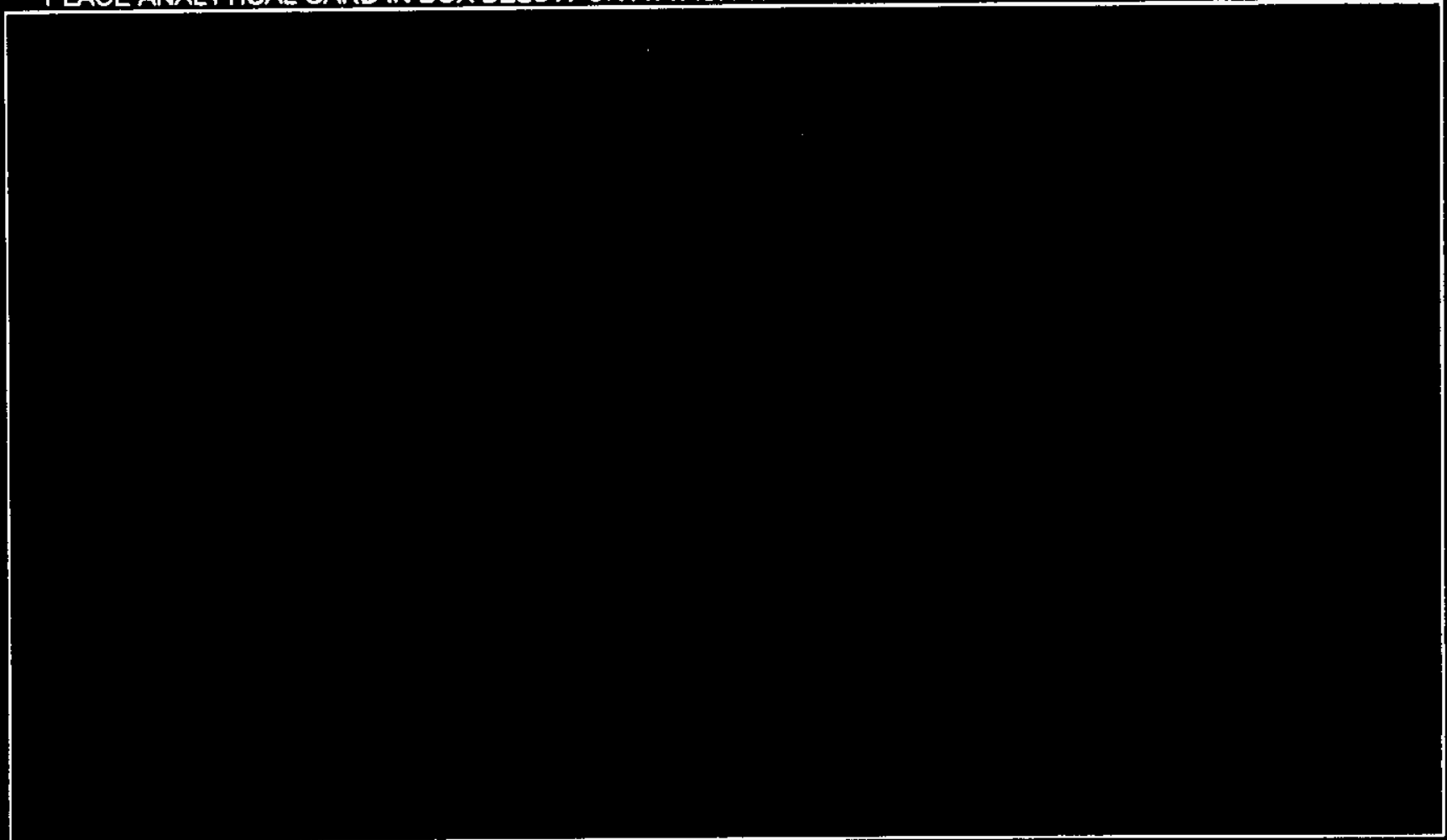

\begin{tabular}{|c|c|c|c|}
\hline \multicolumn{2}{|c|}{ AT : LA-508-101 (D-2) } & \multirow[b]{2}{*}{ STANDARD } & \multirow[b]{2}{*}{ REPUCATE } \\
\hline L & $8-101(A-3)$ & & \\
\hline 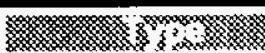 & DETECTOR NUMBER & 2 & 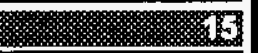 \\
\hline SAWDATV & DISH SIZE 1,2, or 5 & 1. & 2 \\
\hline raporas & TOTAL COUNTS & 68: & 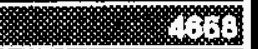 \\
\hline M99 & COUNT TIME IN MINUTES & 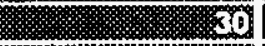 & 30 \\
\hline ; & BACKGROUND in $\mathrm{cPm}$ & (1201 & 0.7 \\
\hline Si & SAMPLE SLE in $\mathrm{mL}$ & 10000 & 10.000 \\
\hline 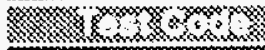 & DILUTION FACTOR & 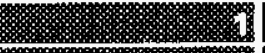 & 1 \\
\hline 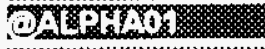 & DICEST DILUTION FACTOR & >ns. & 1 \\
\hline$>1$ & EFFICIENCY FACTOR (EFF) & 0.2380 & 0.2380 \\
\hline TEUाD & LC, Rmax, or Rs,(SAMPLE RATE) as APPROPRIATE & 153.933 & 154.900 \\
\hline mands & Sample Concentration in $\mathrm{MCil}$ & 2.91E-02 & BOOK: \\
\hline 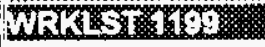 & Replicate Concentration in $\mathrm{IC}$ CL & 2.93E-02 & 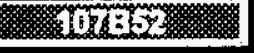 \\
\hline 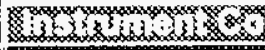 & & & \\
\hline WB26:20 & Average Concentration in ${ }^{\prime} \mathrm{CIL}$ & 2.9226E-02 & \\
\hline$\frac{1}{1}$ & & & \\
\hline KGH. & Rs (Sample Count Rate) $=(\mathrm{TC} / \mathrm{CT}$ ) - BKG & & \\
\hline$\quad 8 \quad 1 \quad 1$ & ALPHA TOTAL $\mu \mathrm{CI} L=$ Rs $* 1000 \mathrm{~mL} \Omega * \mathrm{DF} * \mathrm{DDF} /(\mathrm{EF}$ & $\mathrm{F} * \mathrm{SS} * 2220000 \mathrm{~d}$ & $\mathrm{~m} / \mu \mathrm{Ci})$ \\
\hline $312 \mathrm{Mg}$ & ALPHA TOTAL $\mu \mathrm{Ci} / \mathrm{mL}=$ ALPHA TOTAL $\mu \mathrm{Ci} L / 1000 \mathrm{~mL}$ & & \\
\hline 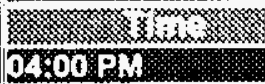 & $\begin{array}{l}\text { Relative Counting Error }=[\text { I (The Square Root of TC }+ \text { BKG } \\
\text { Detection Levels and Less Than Values are determined from P }\end{array}$ & $\begin{array}{l}\text { - CT) / (TC - BKG } \\
\text { rocedure LA-508-0 }\end{array}$ & $\begin{array}{l}\text { CT) }] * 1.96 * 100 \\
2 .\end{array}$ \\
\hline
\end{tabular}

v RESULTS $v$

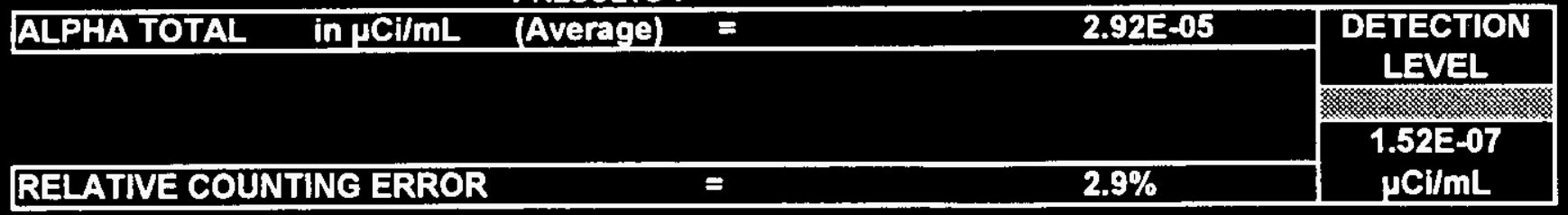

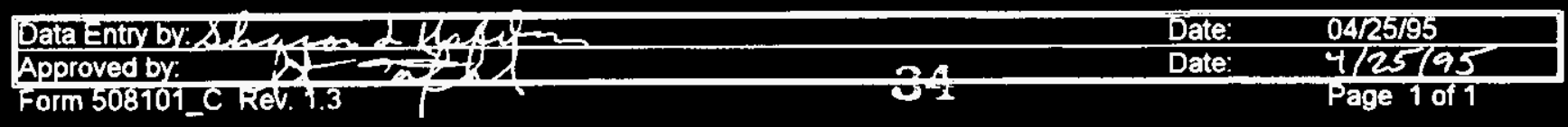




\begin{tabular}{|c|c|c|c|}
\hline \multicolumn{2}{|c|}{ AT : LA-508-101 (D-2) } & \multirow[b]{2}{*}{ BLANK } & \multirow[b]{2}{*}{ REPUCATE } \\
\hline LA-5 & $3-101(A-3)$ & & \\
\hline $13^{2}$ & DETECTOR NUMBER & SI & 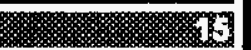 \\
\hline Brivin & DISH SIZE $\quad 1,2$, or 5 & 1 & 2 \\
\hline 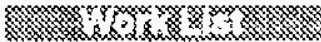 & TOTAL COUNTS & 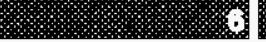 & 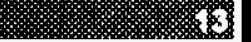 \\
\hline mer & COUNT TIME In MWNTES & 201 & 30 \\
\hline 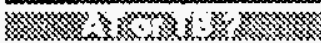 & BACKGROUND in $\mathrm{cPm}$ & On & 0.7 \\
\hline Ar & SAMPLE SIZE in $\mathrm{mL}$ & 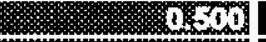 & 0.500 \\
\hline$\ldots$ & DILUTION FACTOR & 济圆 & 1 \\
\hline 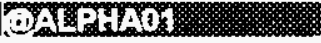 & DIGEST GRAMS of SOLIDS/L & 2021 & 0.4272 \\
\hline$-y_{100}$ & EFFICIENCY FACTOR & 0.2380 & 0.2380 \\
\hline SOY & LC, Rmax, or Rs,(SAMPLE RATE) as APPROPRIATE & 0.356 & 0.356 \\
\hline i.jus & Sample Concentration in $\mathrm{pCi} / \mathrm{g}$ & $3.16 E-03$ & BOOK: \\
\hline 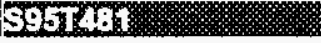 & Replicate Concentration in ychn & $3.16 E-03$ & \\
\hline W & & & \\
\hline ME26072 & Maximum Concentration in pCIg & 3.1583E-03 & \\
\hline 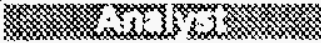 & & & \\
\hline SGH & Rs (Sample Count Rate) $=(\mathrm{TC} / \mathrm{CT})-\mathrm{BKG}$ & & \\
\hline $8^{\mathrm{s}}$ & ALPHA TOTAL $\mu \mathrm{C} / \mathrm{g} g=\mathrm{Rs} * 1000 \mathrm{~mL} \Omega$ " DF $/$ (EFF * Ss & "Dgl $* 2220000 d$ & $\mathrm{~m} / \mathrm{\mu Ci})$ \\
\hline Trangh & & & \\
\hline J4800RT & $\begin{array}{l}\text { Relative Counting Error }=\text { [ (IThe Square Root of TC + BKG } \\
\text { Detection Levels and Less Than Values are determined from } P\end{array}$ & $\begin{array}{l}{ }^{*} \text { CT) } /(\text { (TC - BKG } \\
\text { ocedure LA-508-00 }\end{array}$ & CT) $]=1.96 * 100$ \\
\hline
\end{tabular}

Y RESULTS $v$

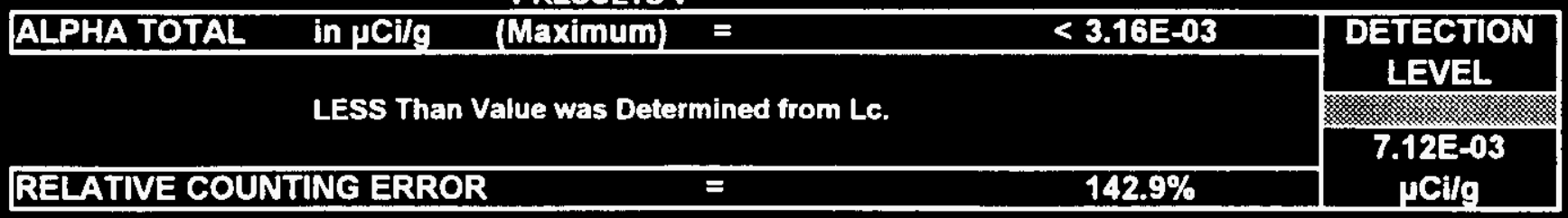

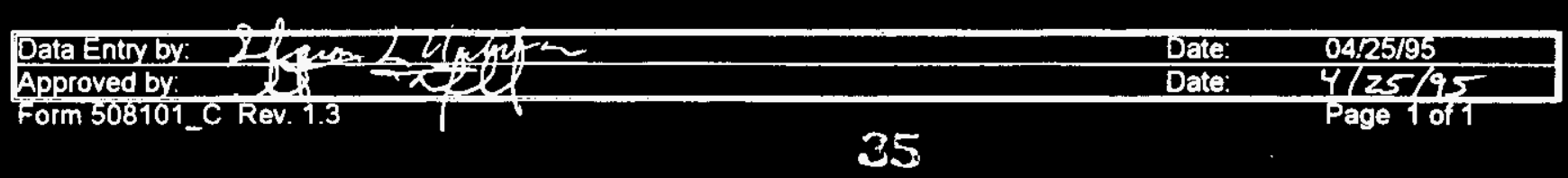




\section{AT : LA-508-101 (D-2)}

\section{LA-548-101 (A-3) SOLIDS}

\begin{tabular}{|c|c|c|c|}
\hline $3^{2}$ & DETECTOR NUMBER & 然 & 13 \\
\hline SAMD2 & DISH SIZE $\quad 1,2$, or 5 & 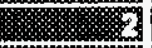 & 2 \\
\hline$\alpha_{1}^{2}$ & TOTAL COUNTS & 80 & 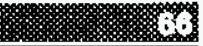 \\
\hline $\mathrm{HOS}$ & COUNT TIME in MINUTES & 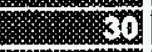 & 30 \\
\hline$x_{1}$ & BACKCROUND in cpm & 00 & 0.7 \\
\hline A & SAMPLE SLE in $\mathrm{mL}$ & 920 & 0.500 \\
\hline 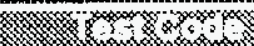 & OLLUTION FACTOR & 1 & 1 \\
\hline WARThis & DIGEST GRAMS of SOLIDSIL & $15 z^{2}$ & 0.4272 \\
\hline $\cos ^{\mathrm{scox}}$ & EFFICIENCY FACTOR & 0.2380 & 0.2380 \\
\hline SOIII & Lc, Rmax or Rs.(SAMPLE RATE) as APPROPRIATE & 0.967 & 1.500 \\
\hline is & Sample Concentration in pClls & 8.57E-03 & BOOK: \\
\hline $305 \mathrm{mat}$ & Replicate Concentration in ucha & 1.33E-02 & \\
\hline
\end{tabular}

\section{W}

W32687? Average Concentration in $\mu \mathrm{CU}$ ?

Sins 3

RCH Rs (Sample Count Rate) = (TC/CT) - BKG

T20195

.

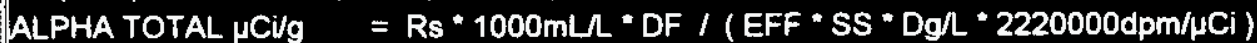

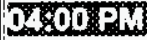

Relative Counting Error = [ |(The Square Root of TC + BKG * CT) / (TC - BKG * CT) ] *1.96*100 Detection Levels and Less Than Values are determined from Procedure LA-508-002.

$\checkmark$ RESULTS $v$

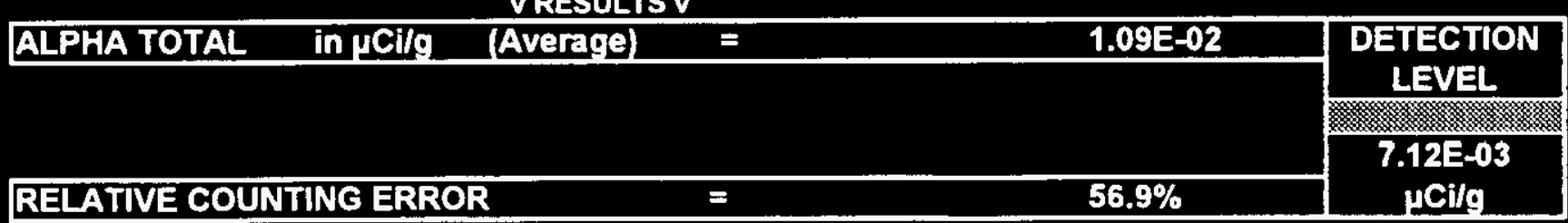

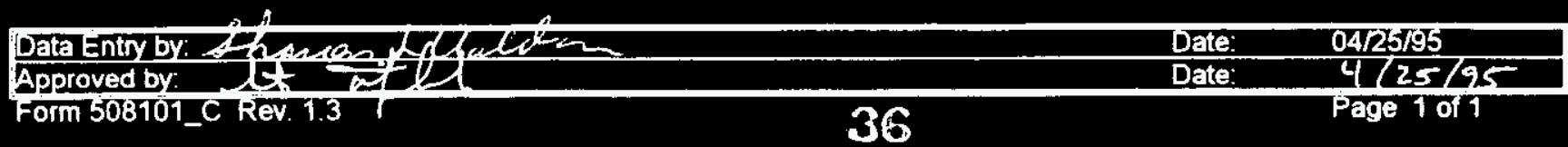


PLACE ANALYTICAL CARD IN BOX BELOW OR ATTACH TRAVELER WHC-SD-WM-DP. 105 , REV. 1

\begin{tabular}{|c|c|c|c|}
\hline \multicolumn{2}{|c|}{ AT : LA-608-101 (D-2) } & & \multirow[b]{2}{*}{ REPLICATE } \\
\hline \multicolumn{2}{|c|}{$L A-548-101(A-3)$} & SAMPLE & \\
\hline 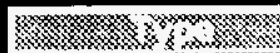 & DETECTOR NUMBER & 10 & 1., \\
\hline 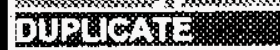 & DISH SIZE 1,2, or 5 & 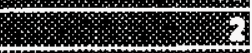 & 2 \\
\hline ry & TOTAL COUNTS & -1 & 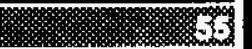 \\
\hline MTe & COUNT TIME in MINUTES & 5 & 30 \\
\hline 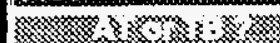 & BACKGROUND in cPm & 200 & 0.7 \\
\hline Ko. & SAMPLE SZE in $\mathrm{mL}$ & 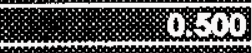 & 0.500 \\
\hline mon & DILUTION FACTOR & 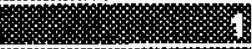 & 1 \\
\hline 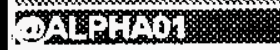 & DIGEST GRAMS Of SOLIDSIL & 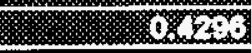 & 0.4296 \\
\hline 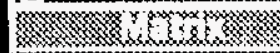 & EFFICIENCY FACTOR & 0.2380 & 0.2380 \\
\hline 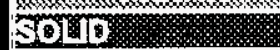 & Lc, Rmax or Rs,(SAMPLE RATE) as APPROPRIATE & 1.233 & 1.133 \\
\hline is & Sample Concentration in $\mathrm{MCL}$ & $1.09 E-02$ & 800K: \\
\hline $355 \pi$ & Replicate concentration in $\mathrm{MCH}$ & Q.995-03 & \\
\hline 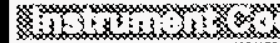 & & & \\
\hline WB26872 & Average Concentration in $\mu \mathrm{C} J$ ? & $1.0427 E-02$ & \\
\hline $\sin 3$ & & & \\
\hline BEH & Rs (Sample Count Rate) & & \\
\hline 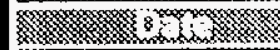 & $=R s * 1000 \mathrm{~mL} \Omega * \mathrm{DF} /(\mathrm{EFF} * \mathrm{Ss}$ & Dg $/ L * 2220000$ & $m / \mu C i)$ \\
\hline $5 \operatorname{arc695}$ & & & \\
\hline$\quad$ & Relative Counting Error $=$ [1(The Square Root of TC + BKG & $\left.{ }^{*} \mathrm{CT}\right) /(\mathrm{TC}-\mathrm{BKC}$ & CT)|I" $1.96 * 100$ \\
\hline T4600 & defermined from & rocedure LA- & \\
\hline
\end{tabular}

V RESULTS V

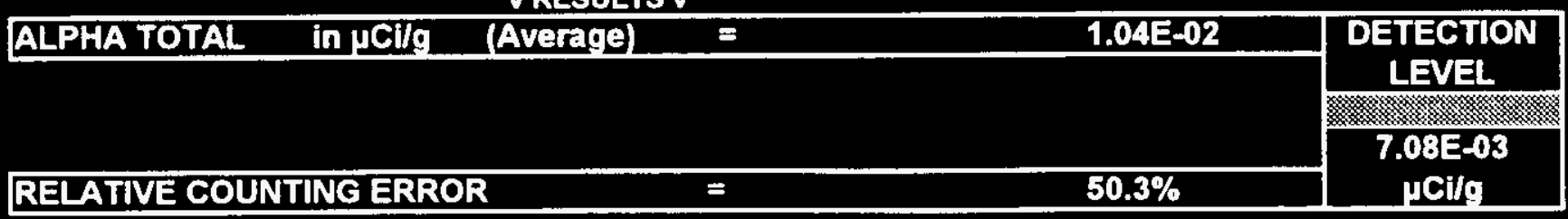

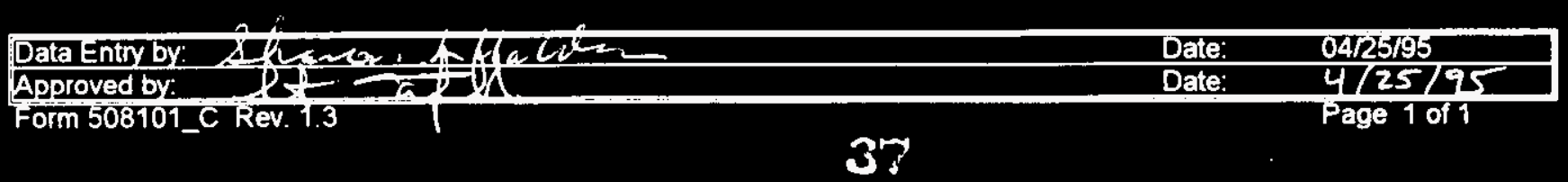


PLACE ANALYTICAL CARD IN BOX BELOW OR ATTACH TRAVELER WHC-SD-WM-DP. 105 REY 1

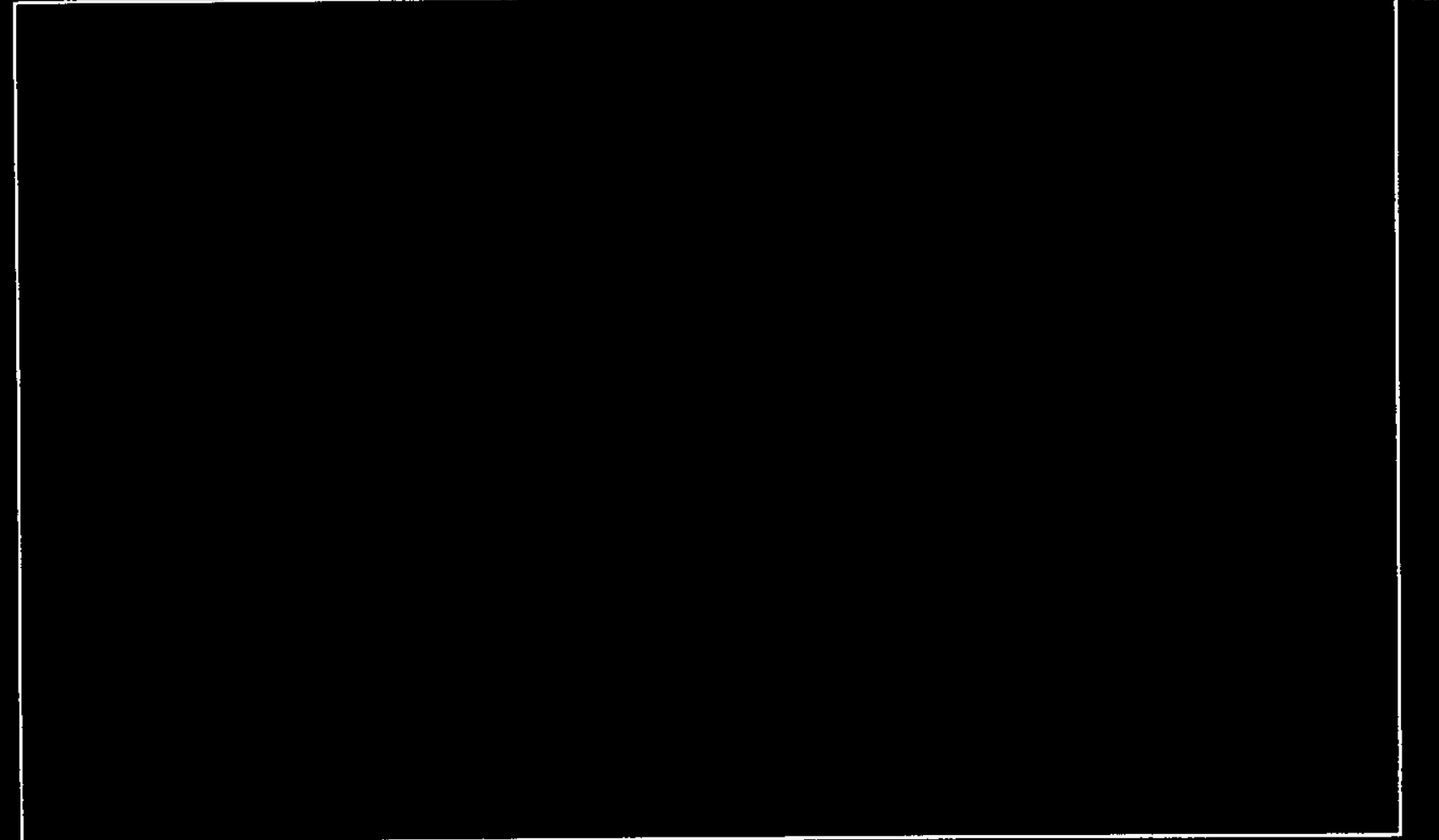

AT : LA-508-101 (D-2)

\begin{tabular}{|c|c|c|c|}
\hline LA-54 & SOLIDS & SPIKE & REPLICATE \\
\hline 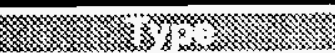 & DETECTOR NUMBER & 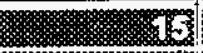 & 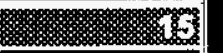 \\
\hline SPIKE & DISH SIZE $\quad 1,2$, or 5 & 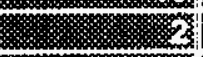 & 2 \\
\hline 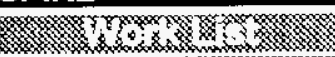 & TOTAL COUNTS & 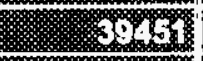 & s. \\
\hline 1190 & COUNT TIME in MINUTES & 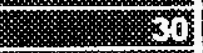 & 30 \\
\hline - & BACKGROUND in cpm & 6 & 0.7 \\
\hline 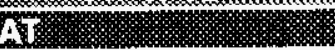 & SAMPLE VOLUME in $\mathrm{mL}$ & 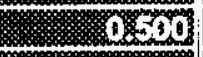 & 0.500 \\
\hline 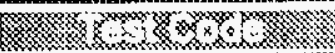 & SAMPLE DILUTION FACTOR & 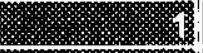 & 1 \\
\hline 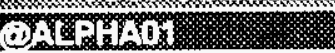 & DIGEST GRAMS of SOLIDS/L & 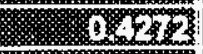 & 0.4272 \\
\hline 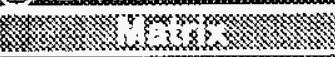 & SPIKE VOLUME in $\mathrm{mL}$ & $0 \times 101$ & 0.100 \\
\hline SOLIB & SPIKE DILUTION FACTOR & S.s.s. & 1 \\
\hline - & SPIKE VALUE in MCIL & 6. & 36.372 \\
\hline S951631: & NSTRUMENT EFFICIENCY FACTOR & 0.238 & 0.238 \\
\hline M & SAMPLE + SPIKE $\mu \mathrm{CI} / \mathrm{g}$ & $1.165+01$ & $1.27 E+01$ \\
\hline WB26622 & AVERACE or MAXIMUM HCIIG from FORM C & 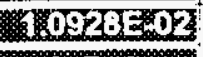 & \\
\hline . & BOOK* & 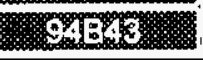 & \\
\hline $\mathrm{BCH}$ & \multirow{5}{*}{\multicolumn{3}{|c|}{ 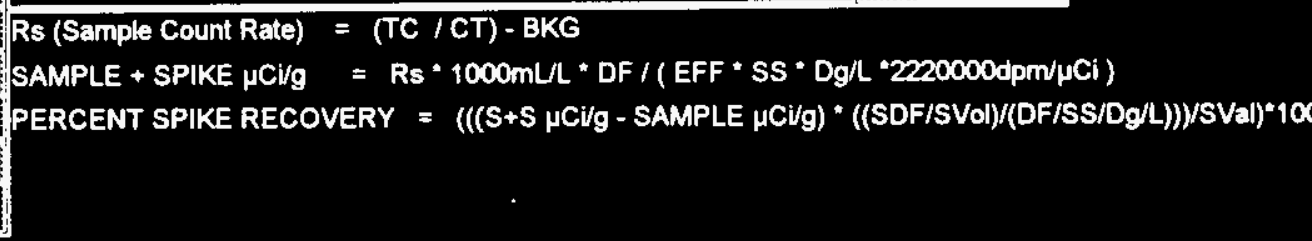 }} \\
\hline 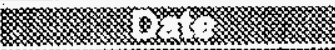 & & & \\
\hline 0.72703 . & & & \\
\hline$\quad \lim _{136}$ & & & \\
\hline 248008026 & & & \\
\hline
\end{tabular}

RESULT AVG. PERCENT SPIKE RECOVERY $=71.6 \%$

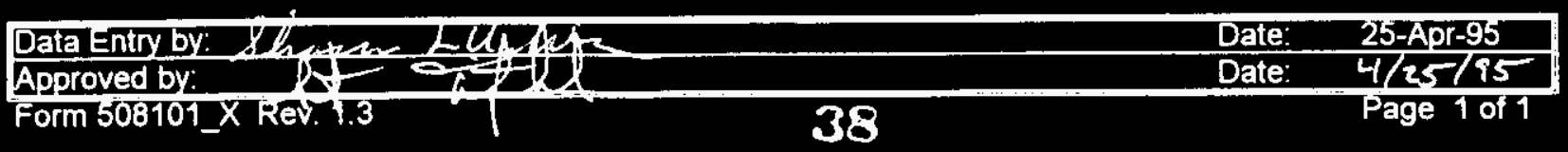


PLACE ANALYTICAL CARD IN BOX BELOW OR ATTACH TRAVELER WHC_SD_WAMDD

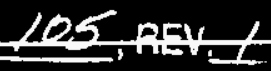

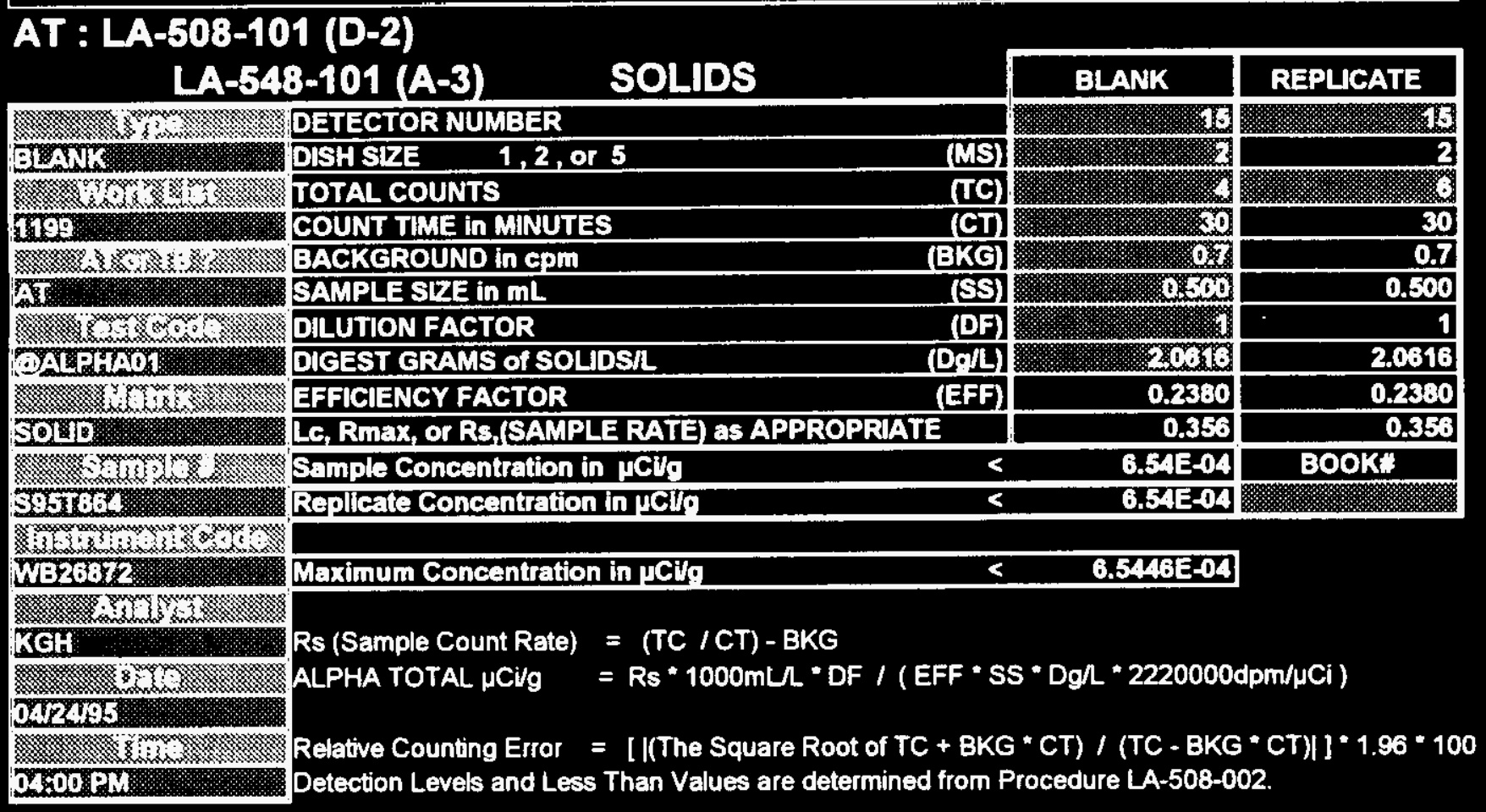

y RESULTS v

\begin{tabular}{|c|c|c|c|}
\hline ALPHA TOTAL & in yCilg (Maximum) = & $<6.54 E-04$ & DETECTION \\
\hline \multicolumn{3}{|c|}{ LESS Than Value was Determined from Lc. } & \\
\hline RELATIVE COU & NG ERROR & $67.9 \%$ & yCilg \\
\hline
\end{tabular}

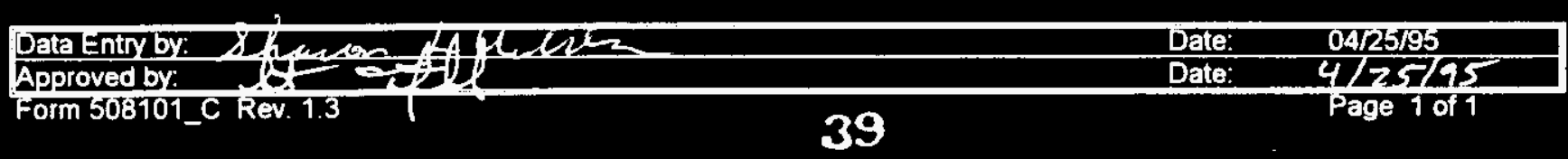


WHC-SD-WM-DP-105, REV 1

\begin{tabular}{|c|c|c|c|}
\hline \multicolumn{2}{|c|}{ AT : LA-508-101 (D-2) } & \multirow[b]{2}{*}{ SAMPLE } & \multirow[b]{2}{*}{ REPLICATE } \\
\hline & $8-101(A-3)$ & & \\
\hline$\quad 2$ & DETECTOR NUMBER & 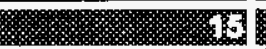 & . \\
\hline 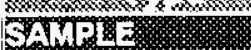 & DISH SIZE 1,2, or 5 & in & 2 \\
\hline 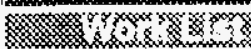 & TOTAL COUNTS & 81 & 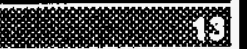 \\
\hline 199 & COUNT TIME in MINUTES & 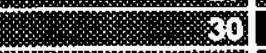 & 30 \\
\hline$\quad 0$ & BACKGROUND in cpm & oxil & 0.7 \\
\hline Ar & SAMPLE STZE in $\mathrm{mL}$ & 1200 & 0.500 \\
\hline 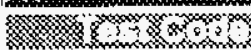 & DLUUTION FACTOR & . & 1 \\
\hline TARTHFY & DIGEST GRAMS Of SOLIDSIL & wastis & 2.0616 \\
\hline Y & EFFICIENCY FACTOR (EFF) & 0.2380 & 0.2380 \\
\hline 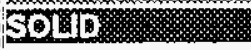 & Le, Rmax or Rs,(SAMPLE RATE) as APPROPRIATE & 0.531 & 0.356 \\
\hline .m. & Sample Concentration in $\mu \mathrm{CHg}$ & $1.07 E-03$ & BOOK: \\
\hline $325016 \% 2$ & Repleate Concentration in pCila & 6.54E-04 & \\
\hline 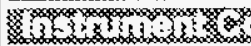 & & & \\
\hline W:26382 & Maximum Concentration in pCls? & 1.0669E-03 & \\
\hline 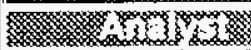 & & & \\
\hline KG & Rs (Sample Count Rate) & & \\
\hline $1 \quad 1 \quad 1$ & ALPHA TOTAL $\mu \mathrm{Ci} / \mathrm{g}=$ Rs * $1000 \mathrm{~mL} \Omega$ * DF $/$ (EFF " Ss & " Dgh * 2220000d & $m / \mu C i)$ \\
\hline $04 \sqrt{24195}$ & & & \\
\hline $04809 \mathrm{RH}$ & $\begin{array}{l}\text { Relative Counting Error }=\text { [ I The Square Root of TC }+ \text { BKG } \\
\text { Detection Levels and Less Than Values are determined from } P\end{array}$ & $\begin{array}{l}\left.{ }^{*} \mathrm{CT}\right) /(\mathrm{TC}-\mathrm{BKG} \\
\text { ocedure LA-508-00 }\end{array}$ & $(\mathrm{CT})]=1.96 * 100$ \\
\hline
\end{tabular}

v RESULTS $\mathrm{v}$

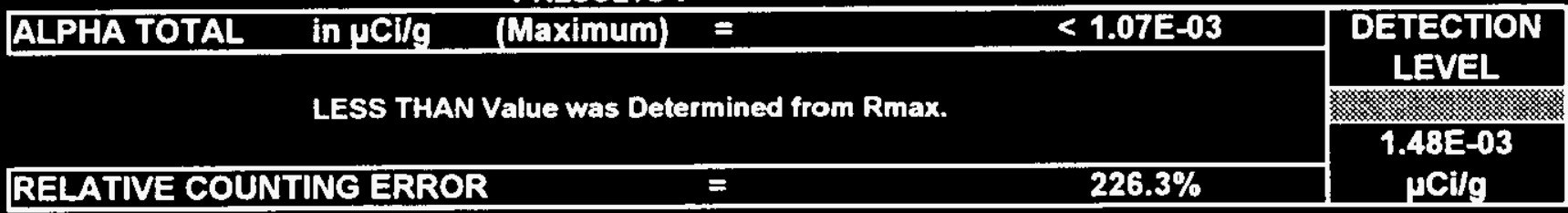

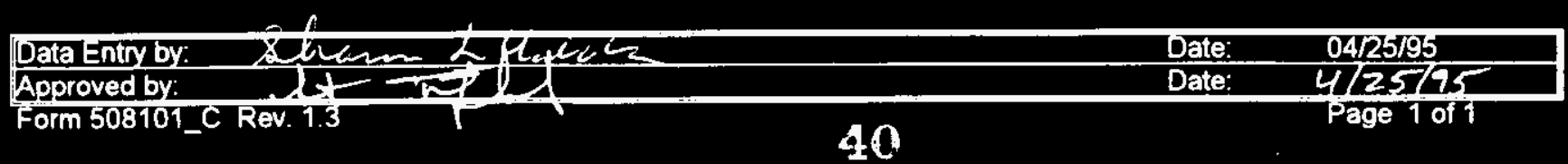




\begin{tabular}{|c|c|c|c|}
\hline \multicolumn{2}{|c|}{ AT : LA-508-101 (D-2) } & \multirow[b]{2}{*}{ SAMPLE } & \multirow[b]{2}{*}{ REPLICATE } \\
\hline LA.54 & $8-101(A-3)$ & & \\
\hline - & DETECTOR NUMBER & 6 & 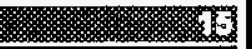 \\
\hline 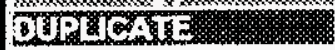 & DLSH SLRE 1,2, or 5 & 3 & 2 \\
\hline rom & TOTAL COUNTS & man & (1) \\
\hline meg & COUNT TIME in MINUTES & 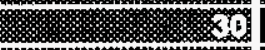 & 30 \\
\hline 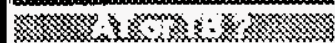 & BACKGROUND in CPM & 201 & 0.7 \\
\hline 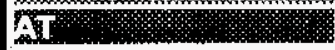 & SAMPLE SIZE in $\mathrm{mL}$ & 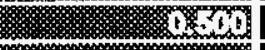 & 0.500 \\
\hline $\cos$ & DILUTION FACTOR & 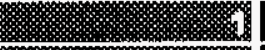 & 1 \\
\hline 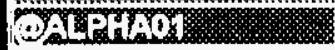 & DIGEST GRAMS of SOLIDSIL & 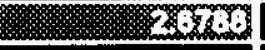 & 2.6788 \\
\hline 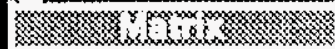 & EFFICIENCY FACTOR & 0.2380 & 0.2380 \\
\hline SOT1 & LC, Rmax, or Rs,(SAMPLE RATE) as APPROPRIATE & 0.356 & 0.356 \\
\hline 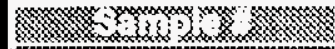 & Sample Concentration in $\mathrm{\mu CH}$ & $5.04 E-04$ & BOOK: \\
\hline $305 \mathrm{~B} \cdot \mathrm{m}$ & Replicate Concentration in ICH/ & 5.04504 & \\
\hline Mink & & & \\
\hline WB26672 & Maximum Concentration in rCHg & $5.0367=04$ & \\
\hline$\ldots$ & & & \\
\hline KaH - & Rs (Sample Count Rate) & & \\
\hline-1 & $=R s * 1000 \mathrm{~mL} \Lambda * \mathrm{DF} /(\mathrm{EFF} * \mathrm{SS}$ & " Dgg * 22200000 & $\mathrm{m} / \mu \mathrm{Ci})$ \\
\hline Trates & & & \\
\hline 500020 & $\begin{array}{l}\text { Relative Counting Error }=[\text { [ }(T \text { The Square Root of } \mathrm{TC}+\mathrm{BKC} \\
\text { Detection Levels and Less Than Values are determined from P }\end{array}$ & "CT) / (TC - BKG & CT) $]]^{* 1.96 * 100}$ \\
\hline
\end{tabular}

$\checkmark$ RESULTS $v$

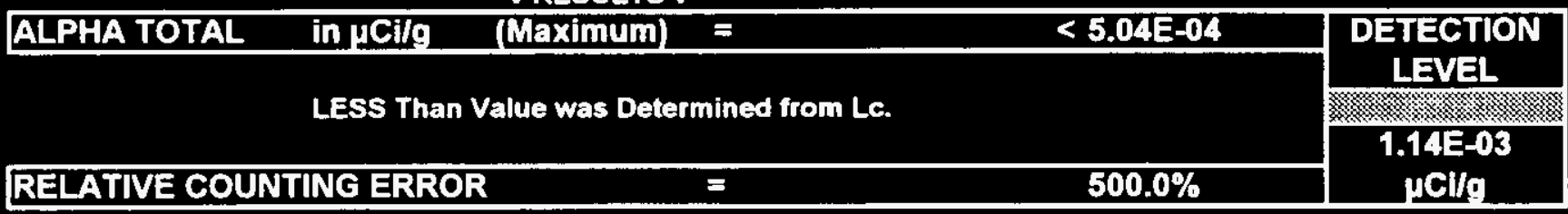

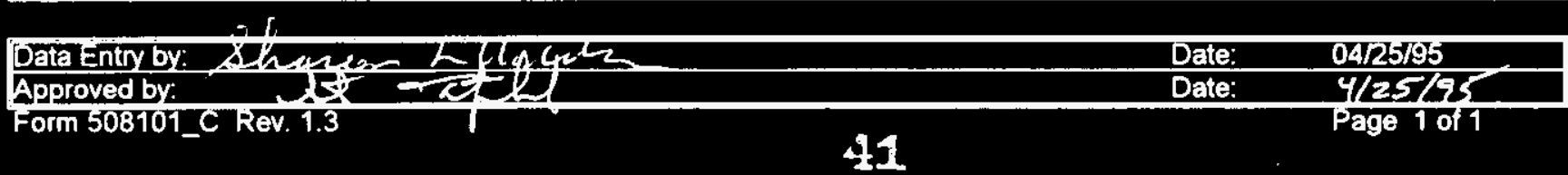


PLACE ANALYTICAL CARD IN BOX BELOW OR ATTACH TRAVELER

\begin{tabular}{|c|c|c|c|}
\hline \multicolumn{2}{|c|}{ AT : LA-508-101 (D-2) } & \multirow[b]{2}{*}{ SAMPLE } & \multirow[b]{2}{*}{ REPLCATE } \\
\hline LA & $8-101(A-3)$ & & \\
\hline$i_{i}$ & DETECTOR NUMBER & 3 & 1. \\
\hline 3AWD20 & DISH SIZE $\quad 1,2$, or 5 & 2 & 2 \\
\hline 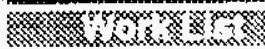 & TOTAL COUNTS & 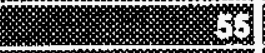 & xis \\
\hline 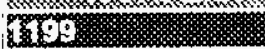 & COUNT TIME IN MINUTES & 3ai & 30 \\
\hline 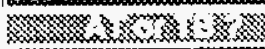 & BACKGROUND in $\mathrm{cPm}$ & 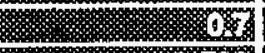 & 0.7 \\
\hline A & SAMPLE SZE in $\mathrm{mL}$ & 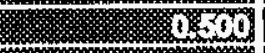 & 0.500 \\
\hline 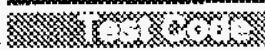 & DILUTION FACTOR & 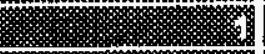 & 1 \\
\hline TARThTS & DIGEST GRAMS of SOLIDSIL & 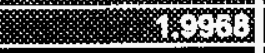 & 1.9968 \\
\hline 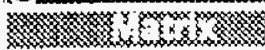 & EFFICIENCY FACTOR & 0.2380 & 0.2380 \\
\hline SOIM] & LC, Rmax, or Rs, (SAMPLE RATE) as APPROPRIATE & 1.133 & 1.433 \\
\hline - & Sample Concentration in $\mathrm{\mu Cilg}$ & 2.15E-03 & BOOK: \\
\hline 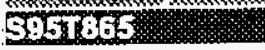 & Replicate Concentration in rClda & $2.72 E 03$ & \\
\hline 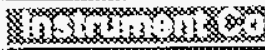 & & & \\
\hline Wez6872 & Average Concentration in ucilg & 2.4328EE-03 & \\
\hline sing & & & \\
\hline Kä & Rs (Sample Count Rate) & & \\
\hline 8 & $=R s * 1000 \mathrm{mLL} * \mathrm{DF} /(\mathrm{EFF} * \mathrm{~s}$ & " Dgl * 2220000 & $\mathrm{m} / \mathrm{NCi})$ \\
\hline Drpugs. & & & \\
\hline 04500PW & $\begin{array}{l}\text { Relative Counting Error = [ | (The Square Root of TC + BKC } \\
\text { Detection Levels and Less Than Values are determined from F }\end{array}$ & "CT) / (TC - BKG & CT) $\mid]^{*} 1.96 *$ \\
\hline
\end{tabular}

V RESULTS V

\begin{tabular}{|lll|c|}
\hline ALPHA TOTAL in UCi/g (Average) $=$ & $2.43 E-03$ & $\begin{array}{c}\text { DETECTION } \\
\text { LEVEL }\end{array}$ \\
\hline RELATIVE COUNTING ERROR & $=$ & & $1.52 E-03$ \\
$\mu C i / g$
\end{tabular}

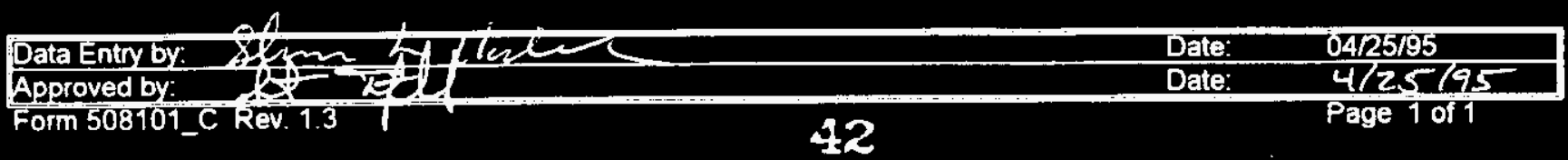


PLACE ANALYTICAL CARD IN BOX BELOW OR ATTACH TRAVELER WHC-SD-W/M-DP. 105 , REV. I

\begin{tabular}{|c|c|c|c|}
\hline \multicolumn{2}{|c|}{ AT: LA-508-101 (D-2) } & \multirow[b]{2}{*}{ SAMPLE } & \multirow[b]{2}{*}{ REPLICATE } \\
\hline & 8-101 (A-3) & & \\
\hline $3 z^{2}$ & DETECTOR NUMBER & 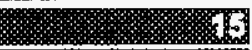 & 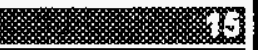 \\
\hline 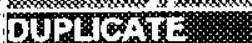 & DISH SIZE 1,2 , or 5 & 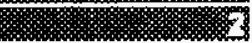 & $\mathbf{2}$ \\
\hline ins & TOTAL COUNTS & sin & xistions \\
\hline$\pi$ Tr & COUNT TIME in MINUTES & ra & 30 \\
\hline 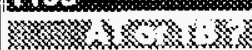 & BACKGROUND in cPm & is & 0.7 \\
\hline s. & SAMPLE SLE in $\mathrm{mL}$ & 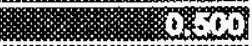 & 0.500 \\
\hline 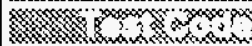 & DILUTION FACTOR & 3 & 1 \\
\hline TARERTH & DIGEST GRAMS Of SOLIDSIL & 23 & 1.992 \\
\hline $\mathrm{y}_{\mathrm{s}}^{\mathrm{mom}}$ & EFFICIENCY FACTOR (EFF) & 0.2380 & 0.2380 \\
\hline SOU: & Le, Rmax, or Rs,(SAMPLE RATE) as APPROPRIATE & 1.533 & 1.133 \\
\hline -imeng & Sample Concentration in $\mu \mathrm{CLO}$ & 2.81E-03 & BOOK: \\
\hline 3851603 & Replicate Concentration in uctra & 2.15E-03 & \\
\hline 1. & & & \\
\hline WB2382 & Average Concentration in $\mu \mathrm{CH}$ & $2.53375-03$ & \\
\hline 13 & & & \\
\hline FGI: & Rs (Sample Count Rate) & & \\
\hline 8 & $=R s * 1000 \mathrm{~mL} / \mathrm{DF} /(\mathrm{EFF} * \mathrm{Ss}$ & " Dgl * 2220000 & $\mathrm{~m} / \mu \mathrm{Ci})$ \\
\hline $0420195 \%$ & & & \\
\hline $0400 \mathrm{PLT}$ & $\begin{array}{l}\text { Relative Counting Error = [ / } \text { The Square Root of TC }+ \text { BKC } \\
\text { Detection Levels and Less Than Values are determined from } P\end{array}$ & $\begin{array}{l}{ }^{*} \text { CT) / (TC - BKC } \\
\text { ocedure LA-508-0 }\end{array}$ & CT) $]]^{* 1.96 * 100}$ \\
\hline
\end{tabular}

v RESULTS v

\begin{tabular}{|lll|c|}
\hline ALPHA TOTAL in $\mu$ Cilg (Average) $=$ & $2.53 E-03$ & $\begin{array}{c}\text { DETECTION } \\
\text { LEVEL }\end{array}$ \\
RELATIVE COUNTING ERROR & $=$ & & $1.53 E-03$ \\
$\mu C I / g$ & $50.3 \%$ & \\
\hline
\end{tabular}

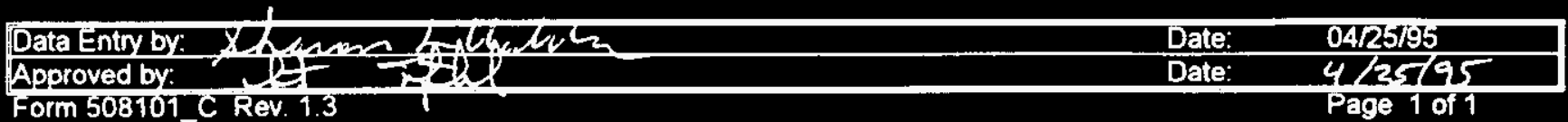


(2) Westinghrouse

Hanford Company

P.O. Box 1970 Richland, WA 99352

PART II

WHC-SD-WM-DP-105, REV. I

WHC-SD-WH-DP-105, REV. 0

ANALYTICAL SERVICES

45-DAY SAFETY SCREEN RESULTS FOR TANK 241-B-112, AUGER SAMPLES, 95-AUG-014 AND 95-AUG-015

Date Printed:

APRIL 26, 1995 
THS PAGE INTENTIONALWX

LBFT BLANK

$7 \pi$ 
WHC-SD WHA-DP. 105, HEV, I

WHC-SD-KH-DP-105, REY. O

TABLE OF CONTENTS

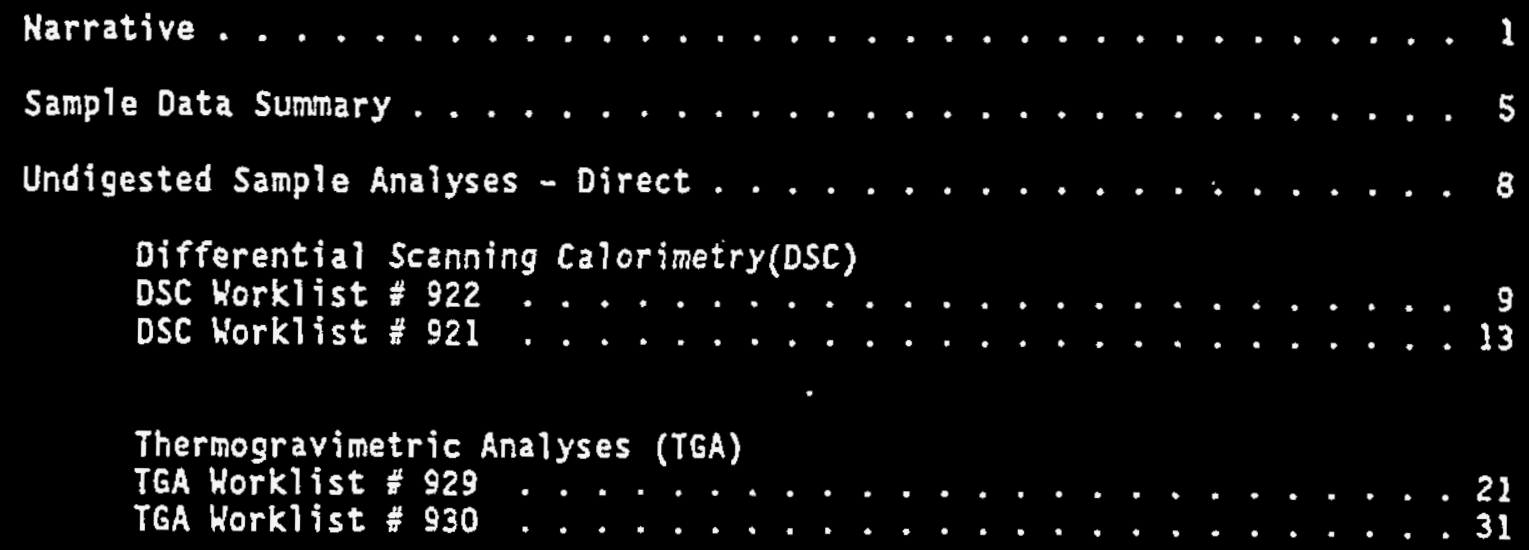

This Document consists of pages 1 through 34 . 


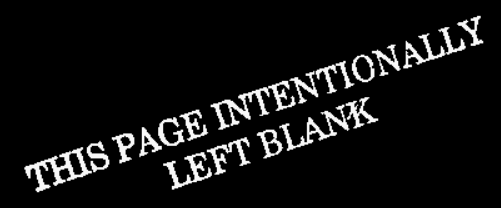


WHCCSD-WM-DP. 105 , REV _L

WHC-SD-HY-DP-105, REY. 0

NARRATIVE

46 


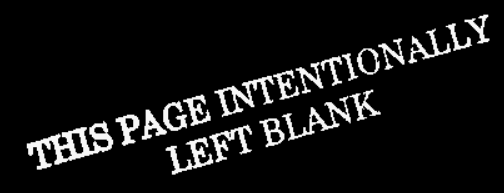




\author{
WHC-SO-WW-DP- 105, REV. I \\ HHC-SD-HH-DP-105, ReV. 0
}

45-DAY SAFETY SCREEN RESULTS FOR TANK 241-B-112, AUGER SAMPLES 95-AUG-014 AND 95-AUG-015

\title{
ANALYTICAL SUMMARY
}

Two auger samples from tank 241-B-112 (B-112) were received in the 222-S Laboratories and underwent safety screening analyses, consisting of differential scanning calorimetry (DSC), thermogravimetric analys is (TGA), and total alpha activity. All results for all analyses (DSC, TGA, and total alpha) were within the safety screening notification limits specified in the Tank Characterization Plan (TCP). No notifications nor secondary analyses were required.

\section{SCOPE}

This document serves as the 45-day report deliverable for the tank B-112 auger samples collected on March 16, 1995 (95-AUG-014 and 95-AUG-015). The 222-S Laboratories received, extruded, and analyzed each sample in accordance with the TCP referenced below. Included in this report are the primary safety screening results obtained from the analyses and copies of all DSC and TGA raw data scans as requested per the TCP. Photographs of the auger samples were taken during extrusion and, al though not included in this report, are available.

\section{SAMPLE RECEIPT, EXTRUSION, AND SUBSAMPLING}

\section{Auger 95-AUG-014}

Auger sample 95-AUG-014 was collected from riser 7 of tank B-112 using a 10 inch auger bit. It was received into the 222-S Laboratories on March 17, 1995 at 1145 and extruded on March 27, 1995. A small amount of drainable liquid dripped off the auger (less than $5 \mathrm{~mL}$ ), but no liquid was recovered for analysis. A gray substance was noticed on flutes 2 and 3 at the top of the auger. These moist solids were subsampled as a possible crust sample $(2.02 \mathrm{~g}$ recovered in vial 6499 ). Het brown solids were subsampled from flutes 4 through 8 (5.70 g subsampled into vial $\$ 6633)$. A total of $7.72 \mathrm{~g}$ of material was recovered from this sample. All analyses required per the TCP were conducted. No archive sample was retained since so little material was collected.

\section{Auger 95-AUG-015}

Auger sample 95-AUG-015 was collected from riser 3 of tank B-112 using a 10 inch auger bit. It was received into the 222-5 Laboratories on March 17, 1995 at 1145 and extruded on March 28, 1995. There was no drainable liquid, and only $2.70 \mathrm{~g}$ of material was recovered. This material (brown solids) was scraped from flutes 3 through 8 of the auger and subsampled into vial $\# 6653$. All analyses required per the TCP were conducted. No archive sample was retained since so littie material was collected. 
WHC-SD-WM-DF-105, HE! !

WHC-SD-WM-DP-105, ReV. 0

SAMPLE IDENTIFICATION INFORMATION

Table 1: Sample Identification

\begin{tabular}{|c|l|l|}
\hline $\begin{array}{c}\text { LABCORE } \\
\text { Sample ID }\end{array}$ & \multicolumn{1}{|c|}{ Customer Sample ID } & \multicolumn{1}{|c|}{ Analyses } \\
\hline S96T000356 & 95 -AUG-014, Riser 7, auger sample & Extrusion \\
\hline S95T000357 & $\begin{array}{l}\text { 95-AUG-014, Riser 7, upper half (crust) } \\
\text { sUbsample }\end{array}$ & DSC/TGA \\
\hline S95T000864 & $\begin{array}{l}\text { 95-AUG-014, Riser 7, upper half (crust) } \\
\text { Subsample fusion/digest }\end{array}$ & Total alpha \\
\hline S95T000452 & $95-A U G-014$, Riser 7, remaining solids subsample & OSC/TGA \\
\hline S95T000481 & $\begin{array}{l}95-A U G-014, \text { Riser 7, remaining solids } \\
\text { fusion/digest }\end{array}$ & Total alpha \\
\hline S95T000453 & $95-A U G-015$, Riser 3, auger sample & Extrusion \\
\hline S95T000454 & 95 -AUG-015, Riser 3, subsample & OSC/TGA \\
\hline S95T000865 & $95-A U G-015$, Riser 3, fusion/digest & Total alpha \\
\hline
\end{tabular}

\section{ANALYTICAL RESUITS}

Analytical results are presented in Tables 2 and 3 , with the applicable notification limits shaded.

\section{DSC (Energetics Content)}

DSC analyses were performed under a nitrogen atmosphere using procedure LA514-113, Rev. B-1. For samples exhibiting exotherms, dry weight basis results were caiculated from the wet weight basis results by using the average percent water by TGA for each sample. Both the wet and dry resuits are given in Tables 2 and 3 .

Sample S95T000357 exhibited a small exotherm (in both sample and duplicate). The average value for the exotherm was $13.80 \mathrm{~J} / \mathrm{g}$ with a relative percent difference (RPD) of $117 \%$, which failed the precision criterion stated in the TCP. The large RPD is due to the presence of an exotherm near the detection limit and lack of homogenization (as explained below for TGA). The average exotherm is less than $3 \%$ of the limit of $481 \mathrm{~J} / \mathrm{g}$. A third sample was anaiyzed for 5957000357 , resulting in an exotherm of $18.7 \mathrm{~J} / \mathrm{g}$. This result does not appear in Table 2, but is provided in the raw data. No other sample exhibited an exotherm. 


\section{WHC-SD-WA-DP-105, REV. I}

MHC-SD-WH-DP-105, ReV. 0

\section{IGA (Moisture Content)}

Weight percent water was performed under a nitrogen atmosphere using procedure LA-560-112, Rev. A-2. Results for samples 595T000357, 595T000452, and \$95T000454 and their duplicates ranged in value from $21 \%$ to $47 \%$ percent water by weight. These results are all above the safety screening action 1 imit of $17 \%$ by weight.

The RPD for sample S95T000357 was $62.4 \%$ (results were 40.19 and 21.08 ). This disparity is most likely due to the fact that the sample was not homogenized. Due to the very small amount of sample available, the material was subsampled directly from the auger into a $20 \mathrm{ml}$ vial without homogenization, which would have resulted in unacceptable sample loss.

A third sample was analyzed for sample $\$ 95 T 000357$, yielding a result of $32.75 \%$ water by weight. A third sample was also analyzed for 595000452 , yieiding a result of $46.96 \%$ water by weight. These results do not appear in Table 2 , but are provided in the raw data.

\section{Total Alpha Activity}

Analyses for total alpha activity were performed on samples 5957000481, S95T000864, and $595 T 000865$. Samples were prepared by fusion using procedure LA-549-141, Rev. C-2, and analyses were performed using procedure $L A-508-101$, Rev. 0-2. A sample duplicate was performed on each sample and a spike was performed on sample S95T000481.

The spike recovery was $71.6 \%$, but no rerun was deemed necessary since the samples were near detection i imits and far below the safety screening action limit of $41 \mathrm{uCi} / \mathrm{g}$.

\section{REFERENCE}

Schreiber, R. D. 1995, WHC-SD-WH-TP-302, Rev, 0, "Tank 241-B-112 Tank Characterization Plan," dated February 6, 1995. 

THS PAGE TNTDNTIO
LETI BLANK

TAIIX 
WHC-SD-WM-DP-105, REV 1

WHC-SD-WM-DP-105, REV. O

SAMPLE DATA SUMMARY

50 


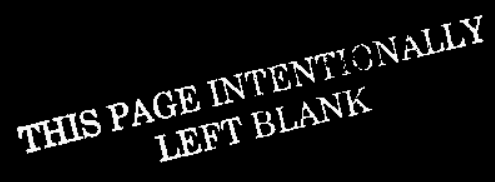



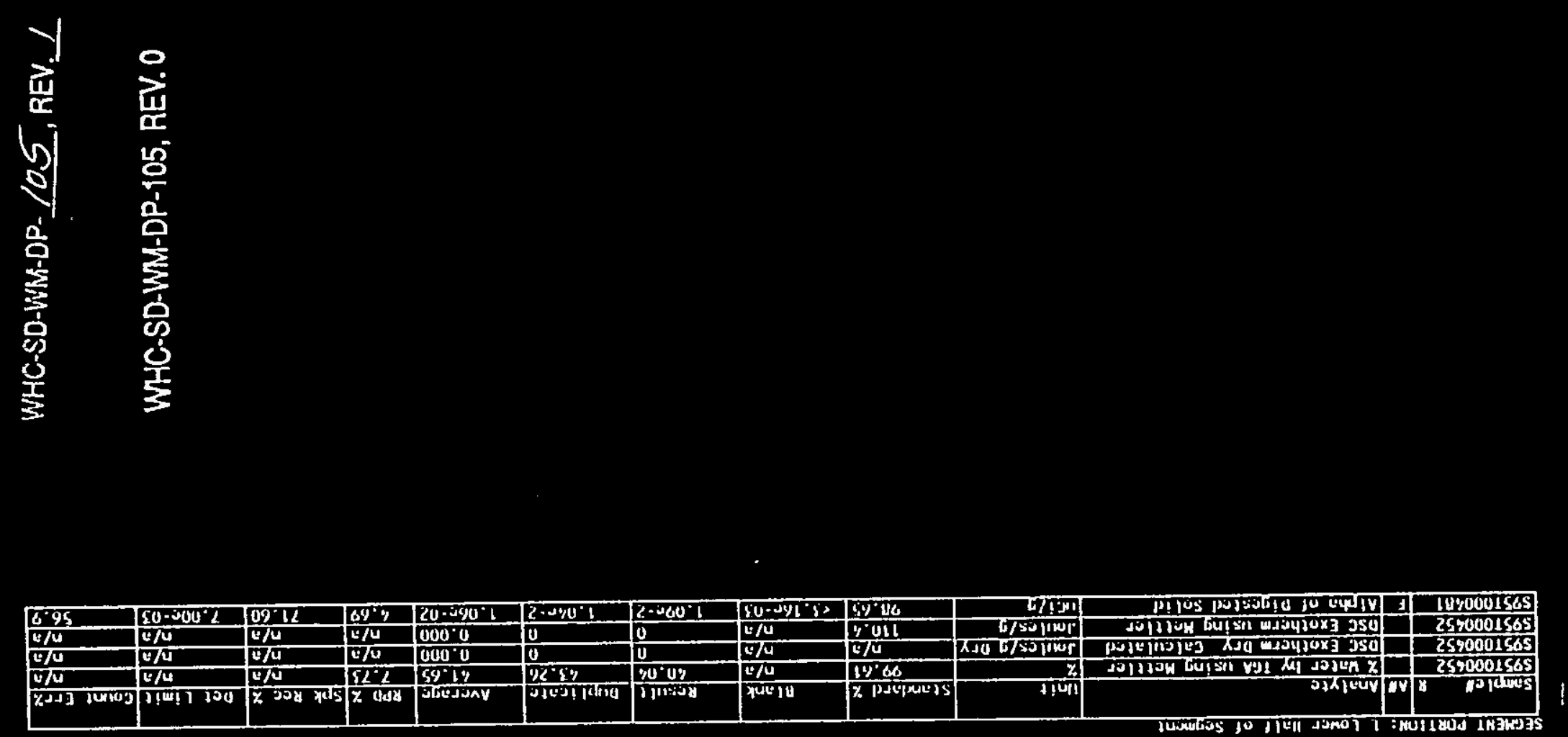

\begin{tabular}{|c|c|c|c|c|c|c|c|c|c|c|c|}
\hline [.922 & $50.500^{\circ}$ & [0/4 & प5u & $0 / \mathrm{M}$ & $y .590 c^{2}$ & $\varepsilon-0<012$ & $80-24,593$ & $50 \div 6$ & $07 ! 31$ & 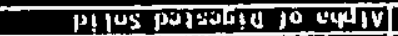 & 2980001569 \\
\hline 870 & EJU & E/u & LII & $08 \mathrm{i}$ & $L^{\cdot S}$ & 6.12 & to/ & 6.011 & G/sojilur & 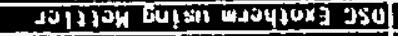 & $\angle 550001568$ \\
\hline EN & Q/u & E/u & 211 & 01.02 & Fo & $b^{\circ} \cdot \bar{L}$ & $0 / \mathrm{u}$ & E/4 & 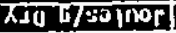 & 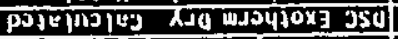 & $\angle 50000150 S$ \\
\hline $0 / 0$ & 世/u & E/T & 129 & SOT & $00^{\circ} 12$ & 61.09 & $0 / 0$ & 1966 & -2 & 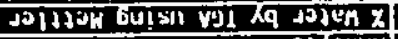 & 4550001505 \\
\hline QIJJ JW105 & गोणी गुण & $x$ 50y $x^{05}$ & $X$ odd & DET JOAY & 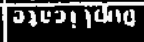 & Intisty & प्रण011 & x pstputis & ग!णी & 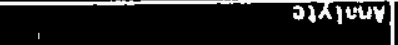 & pojotes \\
\hline
\end{tabular}

2 aาavs

$211-9$

$211-8$ jos sjinsay uados kjojes kea sh

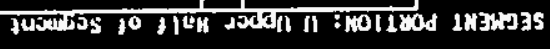

710-onv-ss :y 14akeas

$L$ :yasig

$1 \cdot 2000-4$ 
RISER: 3

TABLE 3

SECHENT \#: 95 -NUE-015

SECHEAr PGRrien: 4 thote segment

Samilen R An Analyte

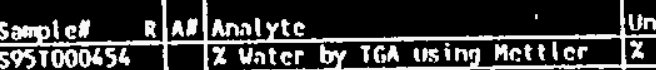

595500045h Dosc Exotheren hry Calculater

5951000454

DSt Exothere using Mattler

Alphin of pirested sol i

standard $x$

\begin{tabular}{|c|c|c|c|c|c|c|c|}
\hline Mlank & Result & monlicats. & Aunrage & $\operatorname{sen} x$ & Sok ReC $X$ & Dat limil & int Erry \\
\hline$n / a$ & 45.57 & 67.14 & 46.36 & 3.39 & $n / 0$ & $n / s$ & $n / a$ \\
\hline$n / 2$ & 0 & 0 & 0.000 & $n / 9$ & $n / a$ & no & $\mathrm{n} / \mathrm{a}$ \\
\hline$n / n$ & 0 & $\overline{\mathbf{n}}$ & 0.000 & $n / n$ & $n / a$ & $n / n$ & $\frac{n / a}{6-1}$ \\
\hline
\end{tabular}
ix.ila 


$$
\text { WHC-SD-WM-DP. 105, REV. I }
$$

WHC-SD-WH-DP-105, REY. O

UNDIGESTED . SAMPLE ANALYSES - DIRECT 


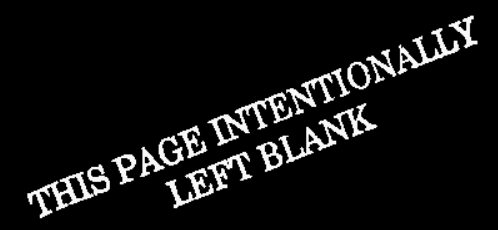


WHC-SD-WHA-DP. LOS, REV

norklistrpt Version 2.0 02/21/95

WHC-SD-WM-DP-105, REV. 0

Ṕage: I

\section{LABCORE Data Entry Template for Worklist\# 922}

Analyst:

Instrument: DSCO1

Book \#/ZN/4-AT

Method: LA-514-113 Rev/Mod $\beta-/$

Worklist Comment: Please run B-112 DSC under N2. bdv

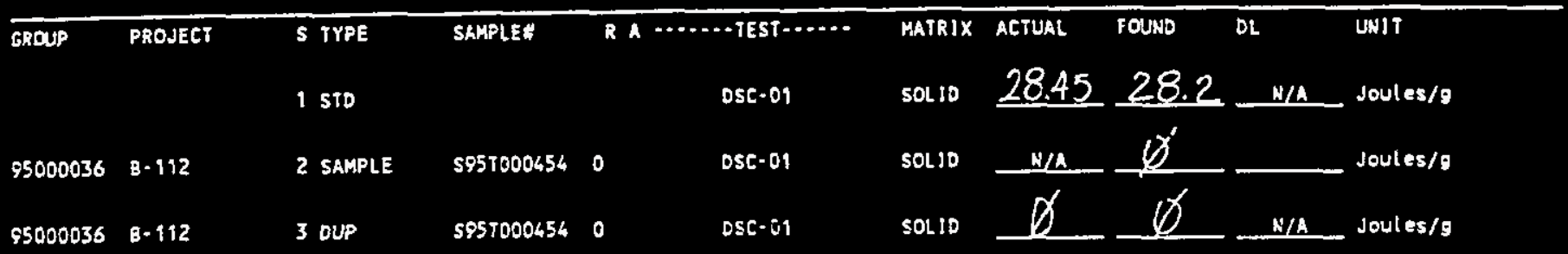

\section{Final page for worklist \# 922}

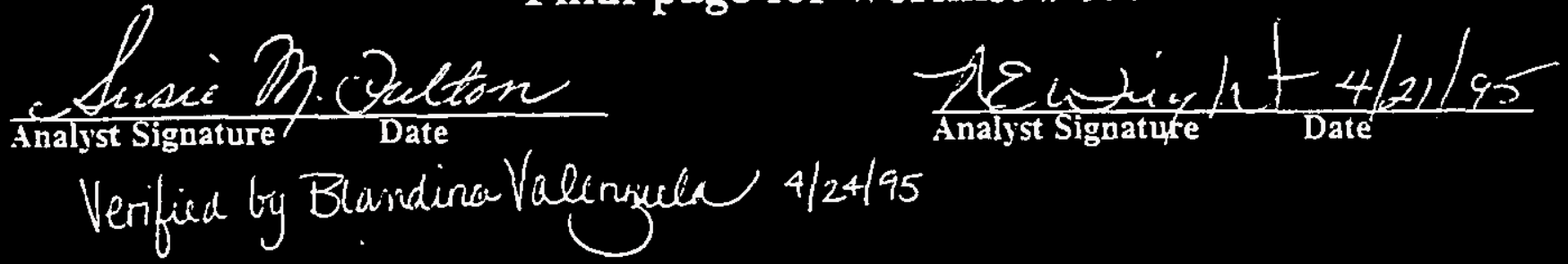

Data Entry Comments: Sample 5957000454 produced tuso endotherms ou at $121.3^{\circ} \mathrm{C}$ with a decte $H$ of $1117.6 \mathrm{~J} / \mathrm{g}$ and the surad at $211.0^{\circ} \mathrm{C}$ with e dele $\mathrm{H}$ of $4.5 \mathrm{~J}$

Units shown for $Q C$ (SPK \& STD) may not reflect the actual units. $D L=$ Detection Limit, $S=$ Worklist Slot Number, $R=$ Replicate Number, $A=$ Aliquot Code. 
STGUTURE BGLOW RERTESGNS CHEMICAL TECINOLOGIST/CIIENIST THITT

CONPLOARD/VERIFIDD TIE CALTBRATION/ANALYSIS ON PAGES S5 TO 27.

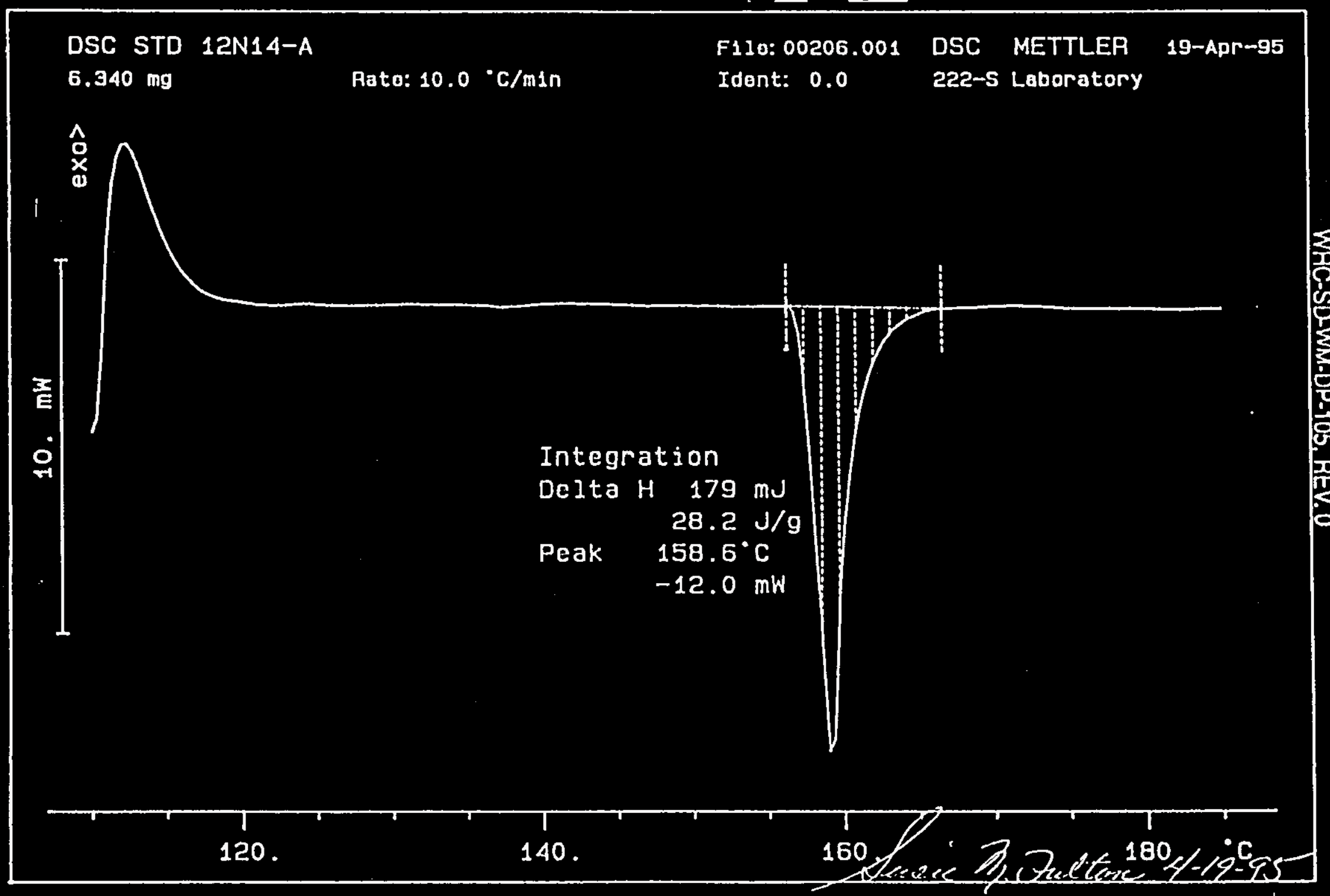




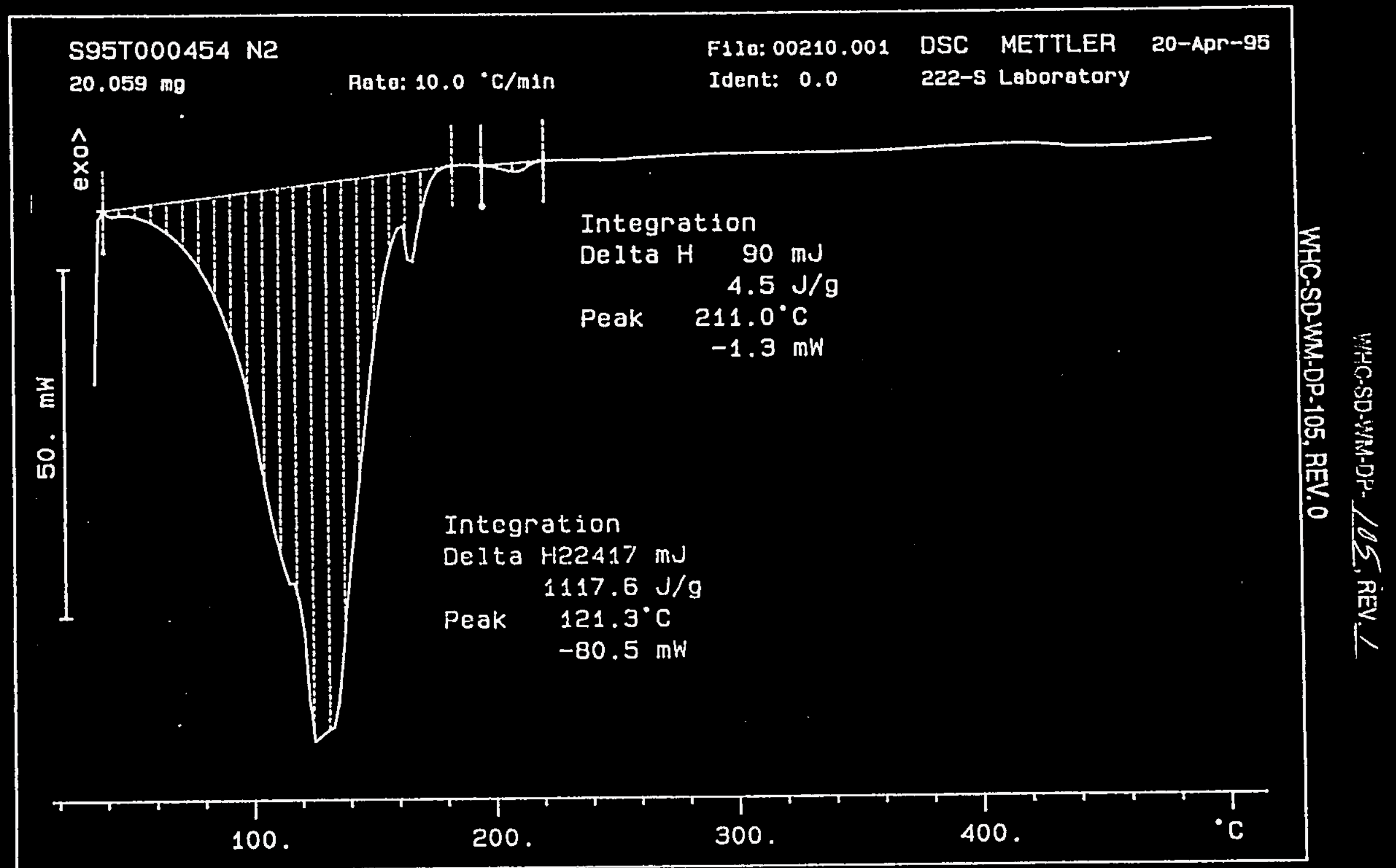




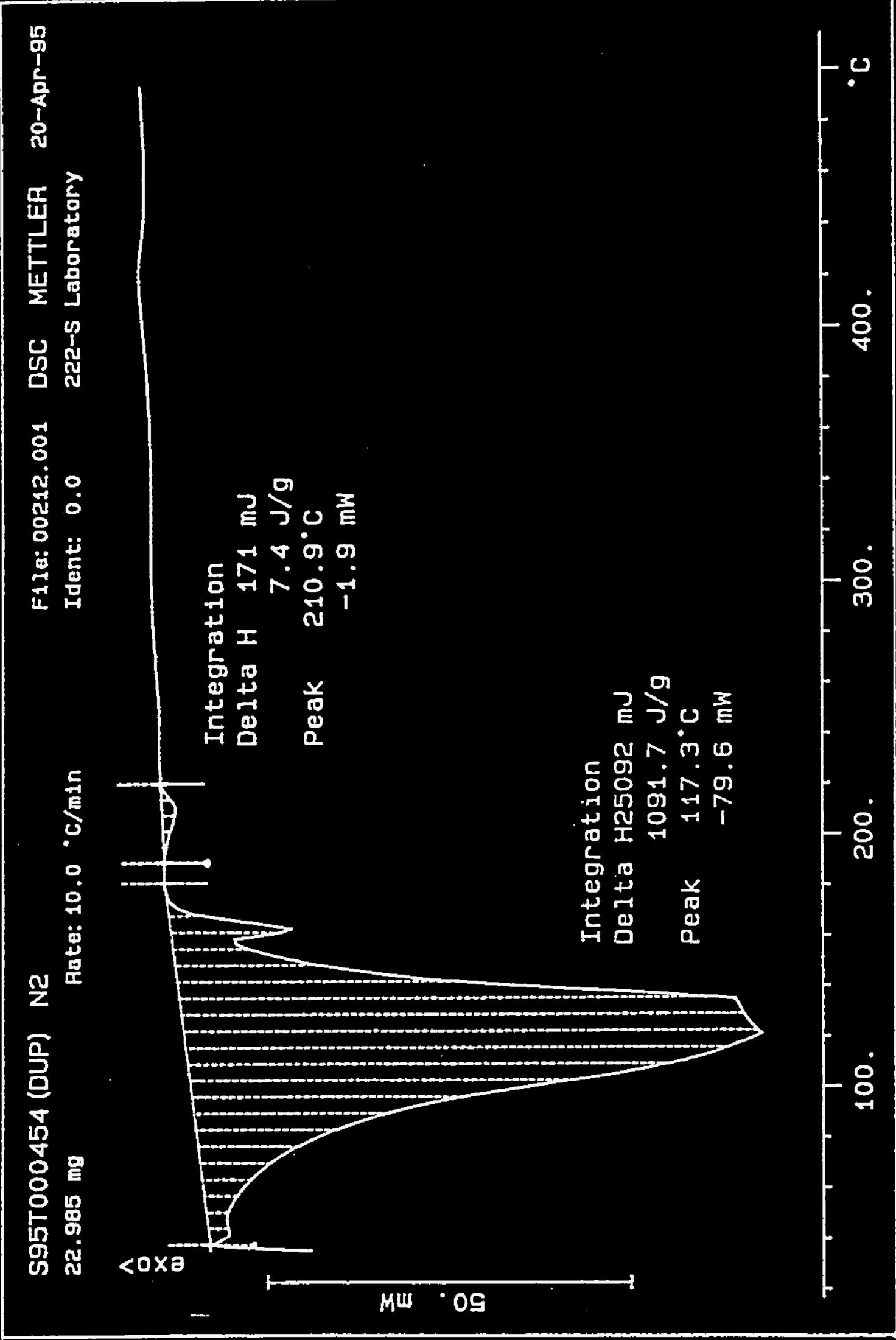




\section{LABCORE Data Entry Template for Worklist\# 921}

Analyst: DWS Instrument: DSC01 Book \# 12N14-A

Method: LA-514-113 Rev/Mod B-1

Worklist Comment: Please run B-112 DSC under N2. bdv

\begin{tabular}{|c|c|c|c|c|c|c|c|c|c|c|}
\hline GROJP & PROJECT & 5 TYPE & SAMPLE\# & $R A$ & 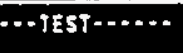 & MATRIX & ACTUAL & FOUND & DL & UNIT \\
\hline & & 1570 & & & DSE-01 & SOL10 & 28.45 & 31.0 & N/A & Joules $/ 9$ \\
\hline 95000032 & B-112 & 2 SAMPLE & $\$ 951000357$ & 0 & Esc-01 & SOL ID & N/A & 21.9 & & Joules/g \\
\hline 95000032 & 8-112 & 3 DUP & 5957000357 & 0 & OSE. 01 & SOLID & 2.1 .9 & 5.7 & N/A & Joules/s \\
\hline & & $45 T 0$ & & & DSC-01 & SOLID & 28.45 & 31.4 & N/A & Joules $/ 9$ \\
\hline 95000032 & B-112 & 5 DUP2 & $\$ 957000357$ & 0 & DSC- 01 & SOLID & 01.9 & 18.7 & N/A & Joules/g \\
\hline 95000032 & 8-112 & 6 SAMPLE & $\$ 951000452$ & 0 & DSC-01 & SOL ID & N/A & $\varnothing$ & & Joules $/ \mathrm{g}$ \\
\hline 95030032 & B-112 & 7 DUP & $\$ 957000452$ & 0 & $0 \leq c .01$ & SOLID & $\varnothing$ & $\phi$ & $N / A$ & Joules/9 \\
\hline
\end{tabular}

Final page for worklist \# 921

$\frac{\text { Seocettached Lor Simatures }}{\text { Analyst Signaturet }}$

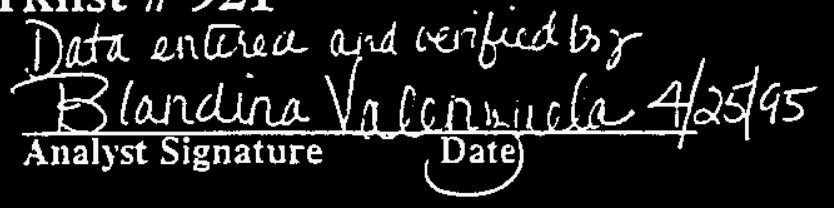

Dara Entry Comments: $\$ 957000357$ produced two endotherms one at $115.3^{\circ} \mathrm{C}$

With a delta $H$ of $883.0 \mathrm{~J} / \mathrm{g}$, and the second at $258.1^{\circ} \mathrm{C}$ with a delta H of $96.9 \mathrm{~J} / \mathrm{g}$. The SHd and DUP2 for 5957000357 was per formud later, cince the chenist was able to lock at the results.

Uniss shown for $Q C$ (SPK \& STD) may notrefiect the octual upirs. DL = Detection Limit, $S$ = Worklist Slor Number,

$R=$ Replicare Number, $A=$ Aliquot Code. $\$ 951000452$ produced $a-d o$ an endo therm

of $947.4 \mathrm{~J} / \mathrm{g}$ t/25/45 Epstios 
WHC-SD-WM-DP- 105, REV. I

worklistrpt Version 2.0 02/2I/95

04/03/95 12:51

\section{LABCORE Data Entry Template for Worklist\# 921}

Poge: $\quad I$

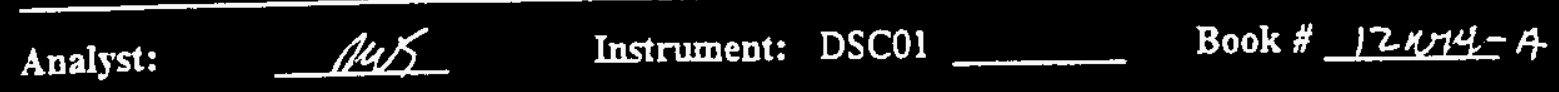

Method: LA-514-113 Rev/Mod $B-1$

Worklist Comment: Please run B-112 DSC under N2. bdv

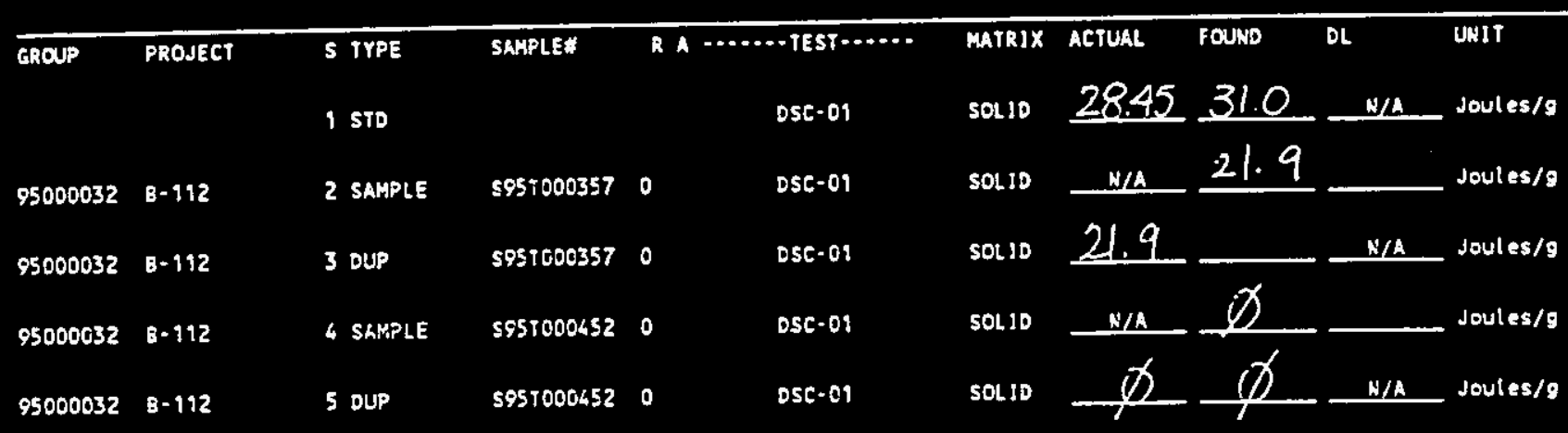

\section{Final page for worklist \# 921}

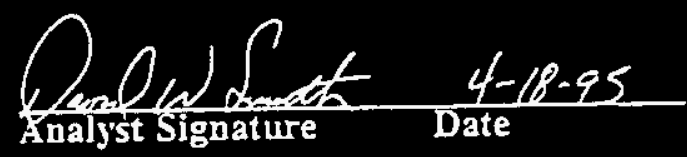

Analyst Signature Date

Data Entry Comments:

Unizs shown for $Q C$ (SPK \& STD) may notreflect the actual uniss. $D L=$ Decetion Limit, $S=$ Worklist Slot Number, $R=$ Replicase Number, $A=$ Aliquor Code. 


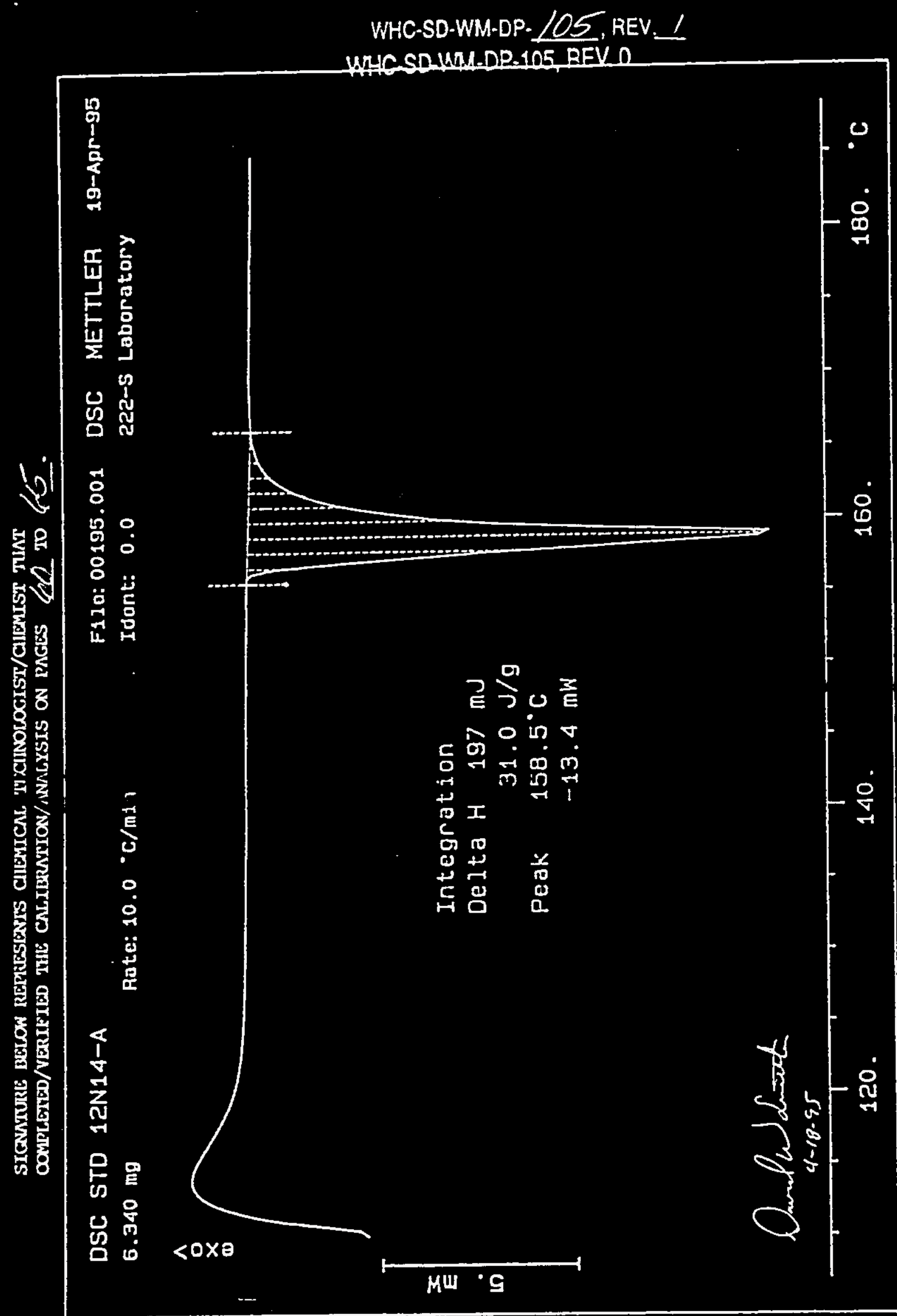

$60^{-}$ 


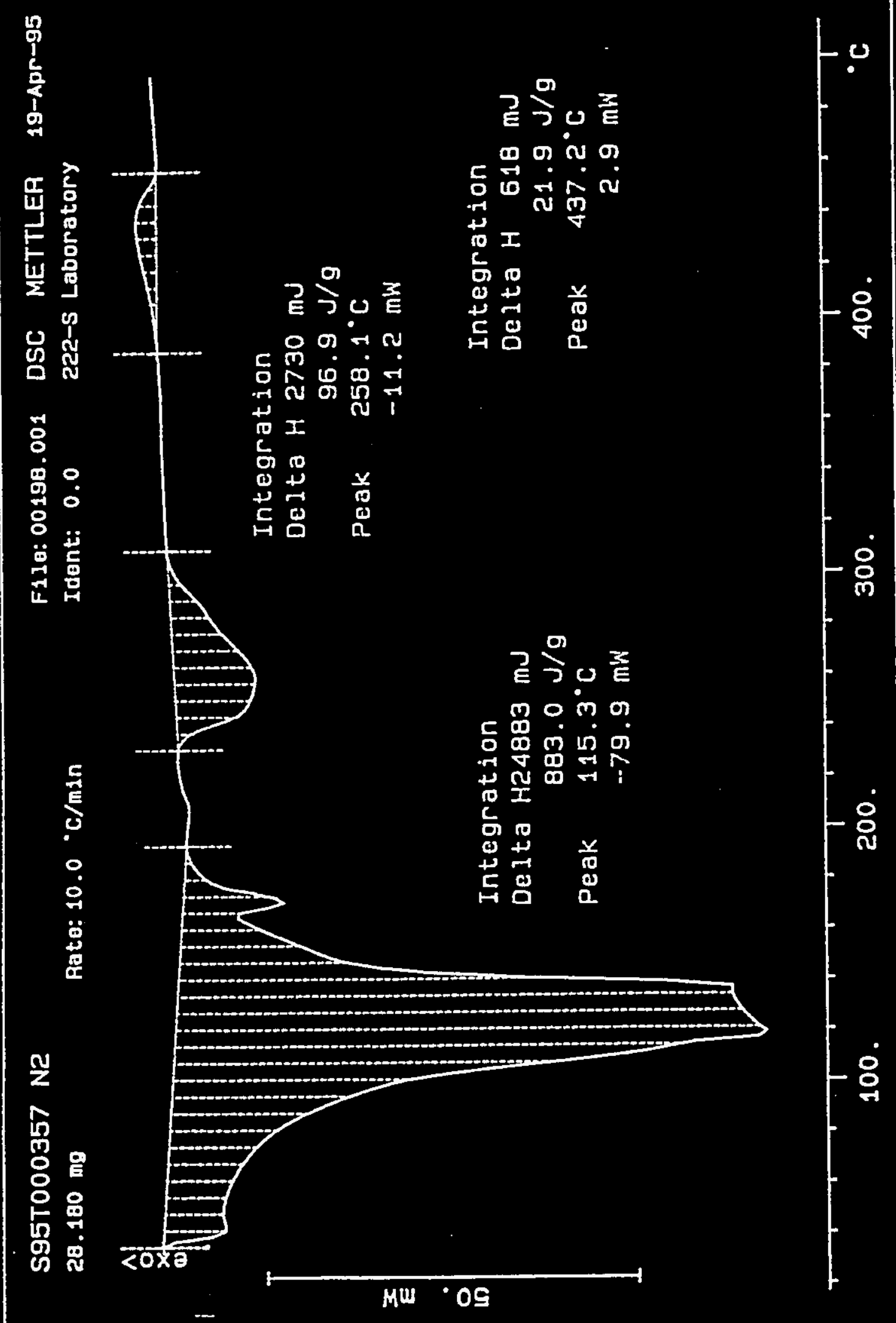




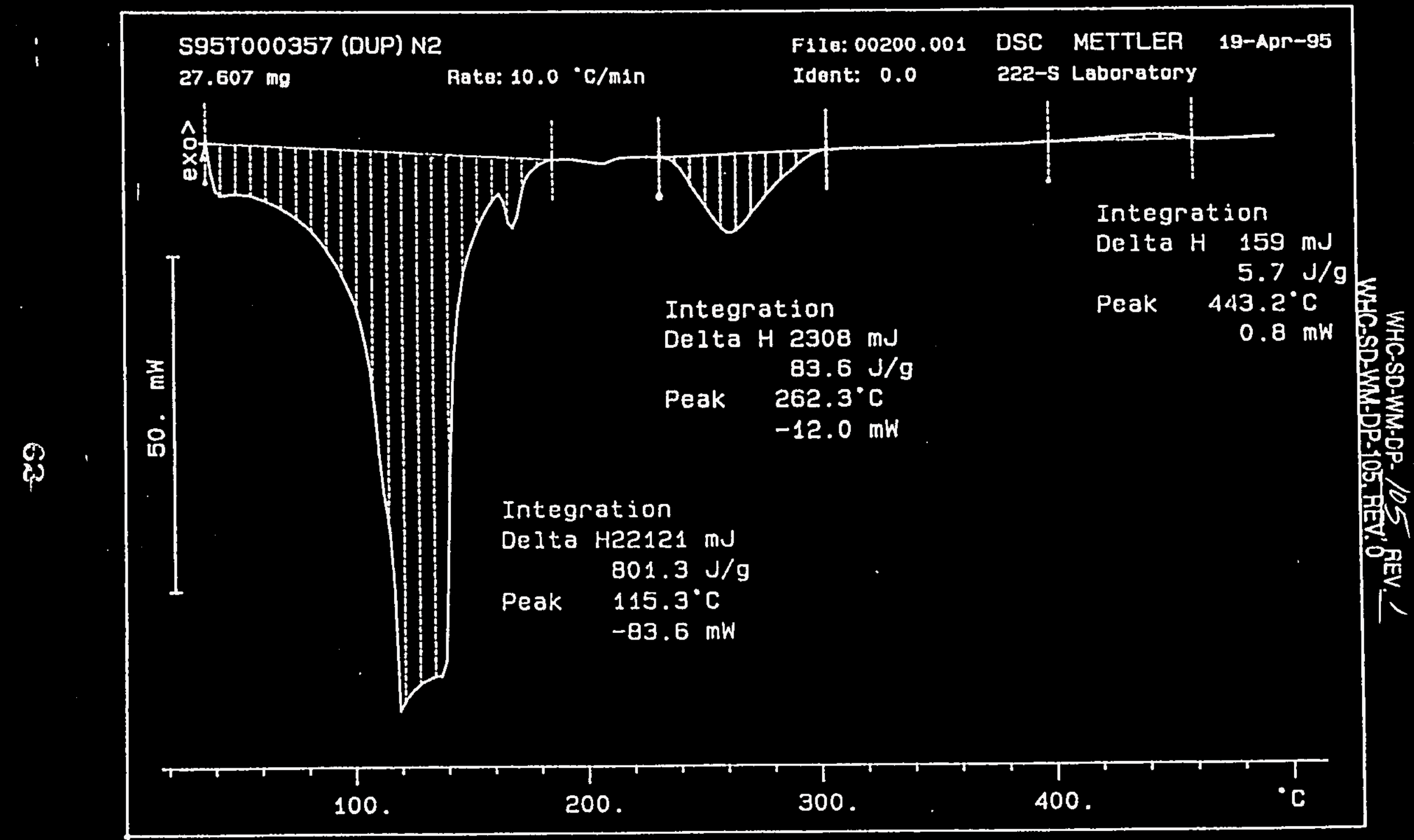




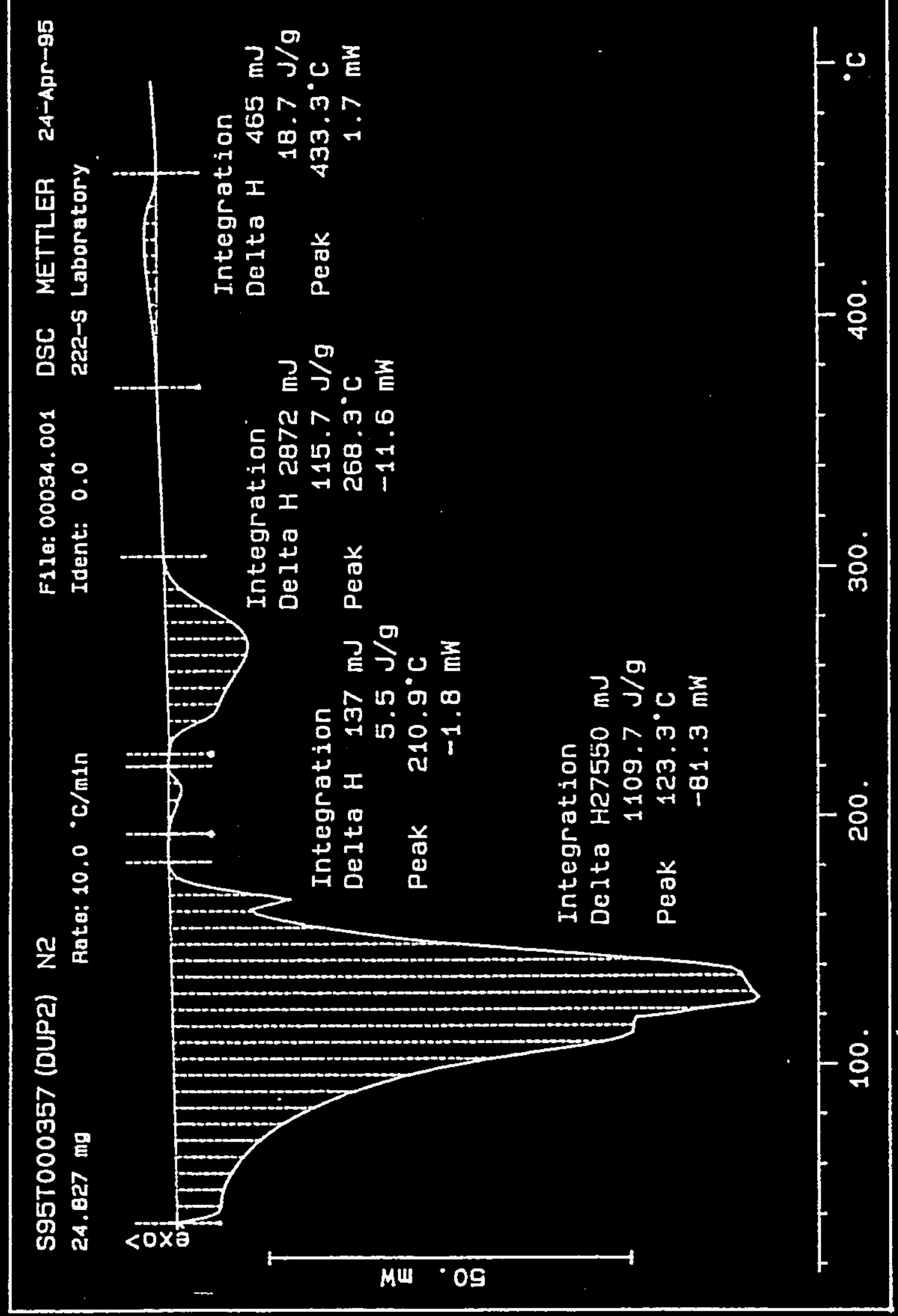

63 
WHC-SD-WM-DP. 105, REV. I UHCSDUM DP $105, R E V O$

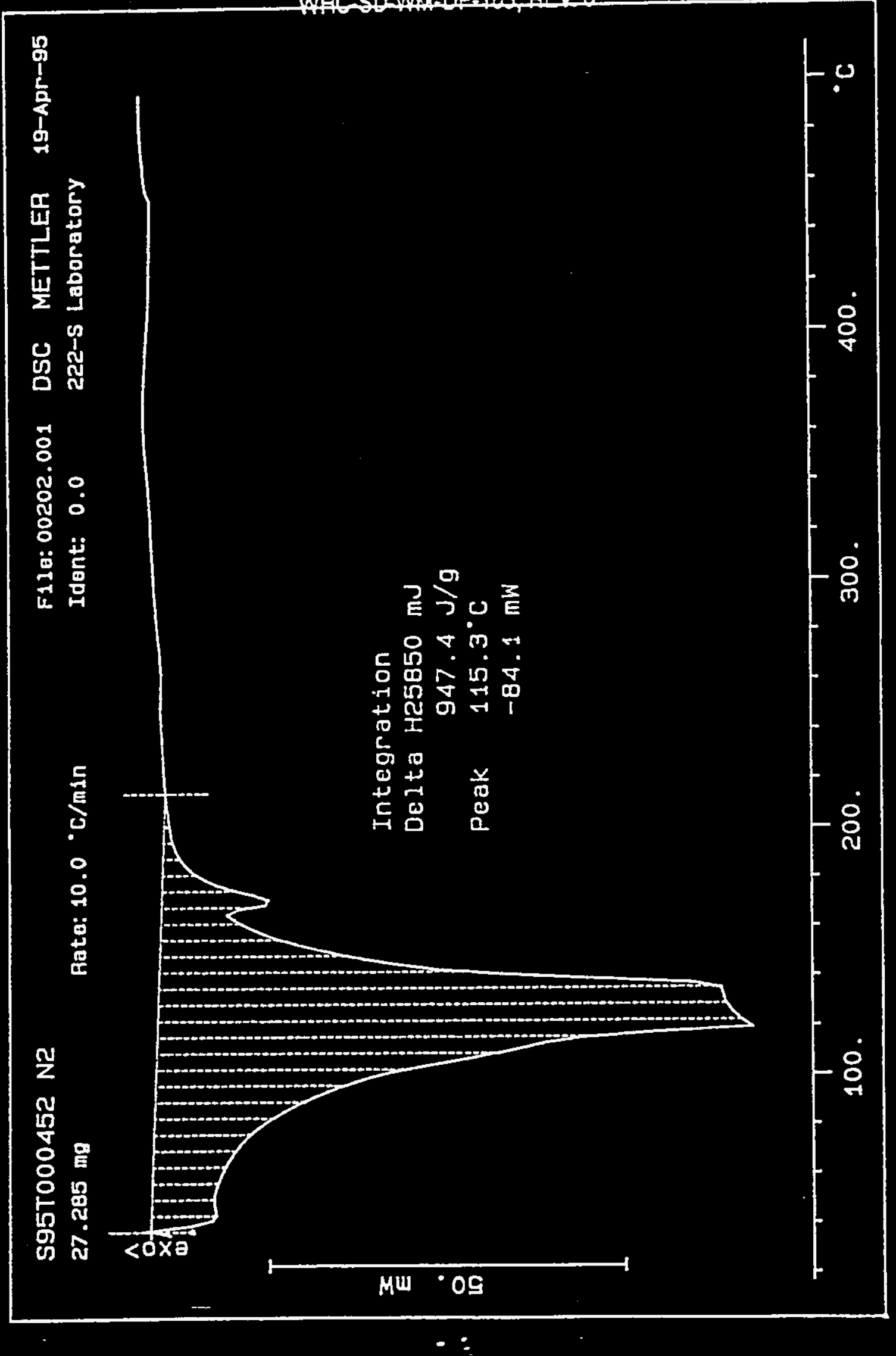




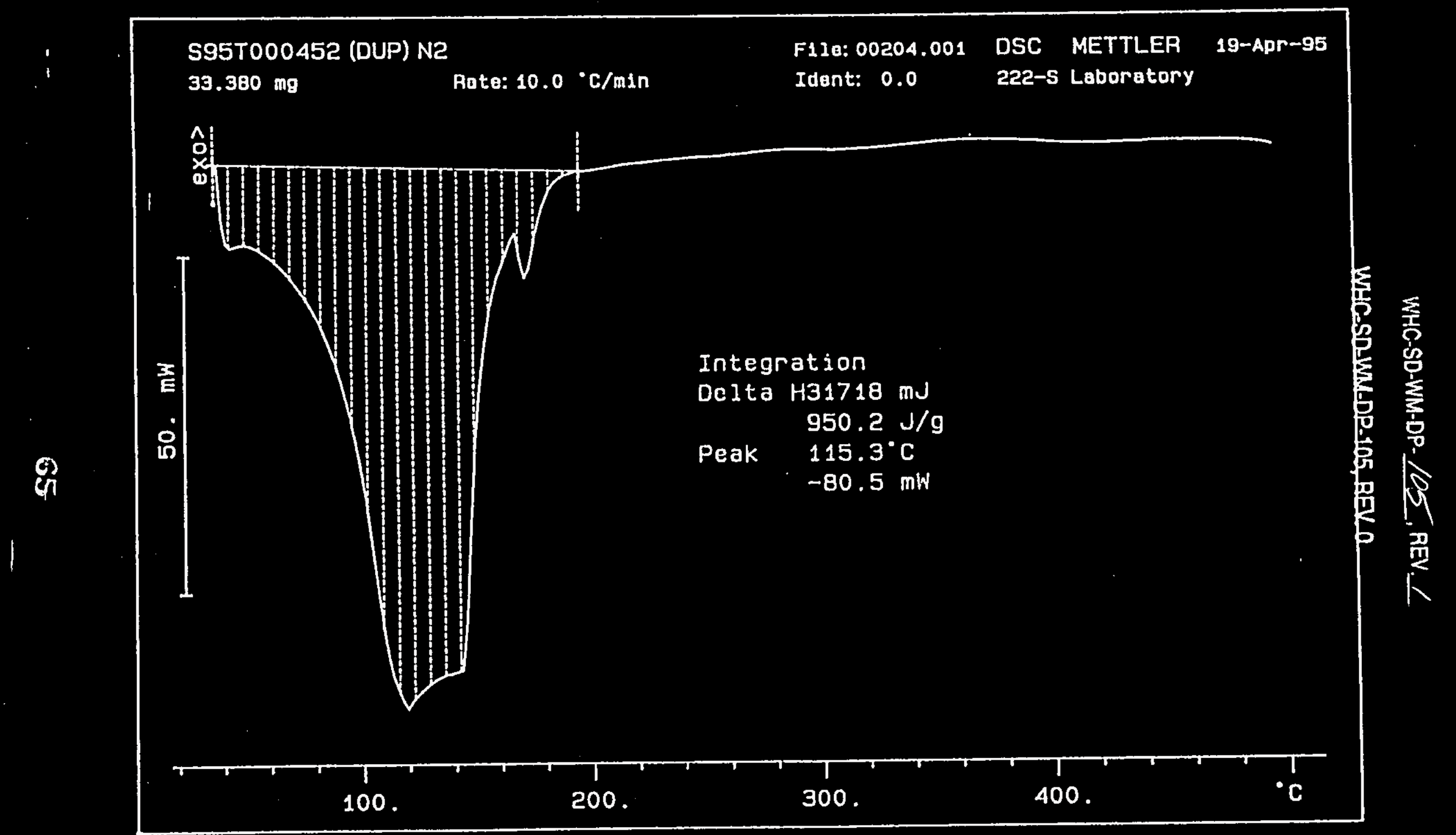




\section{LABCORE Data Entry Template for Worklist\# 929}

Analyst: $\quad$ DWS $\quad$ Instrument: TGA01 _ Book $\# 420184$

Method: LA-560-112 Rev/Mod A-2

Worklist Comment: Please run B-112 TGA under N2. bdv

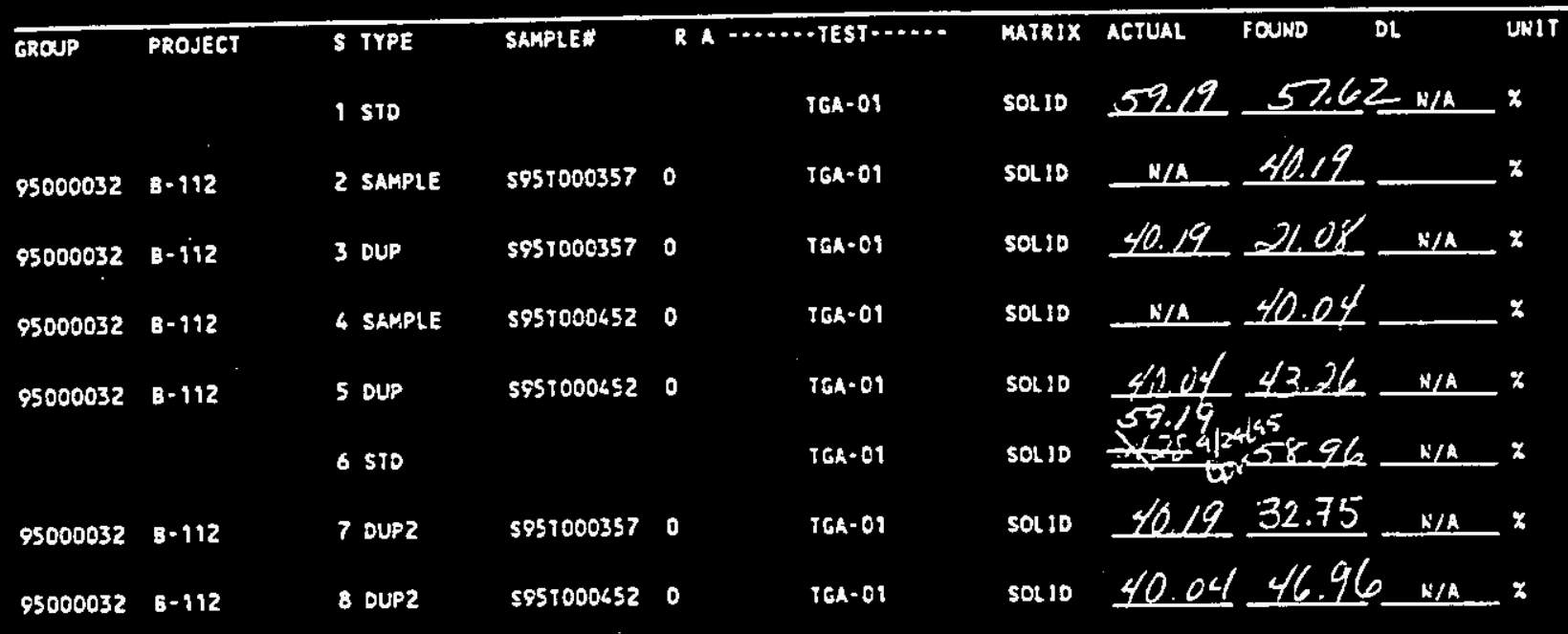

\section{Final page for worklist \# 929}

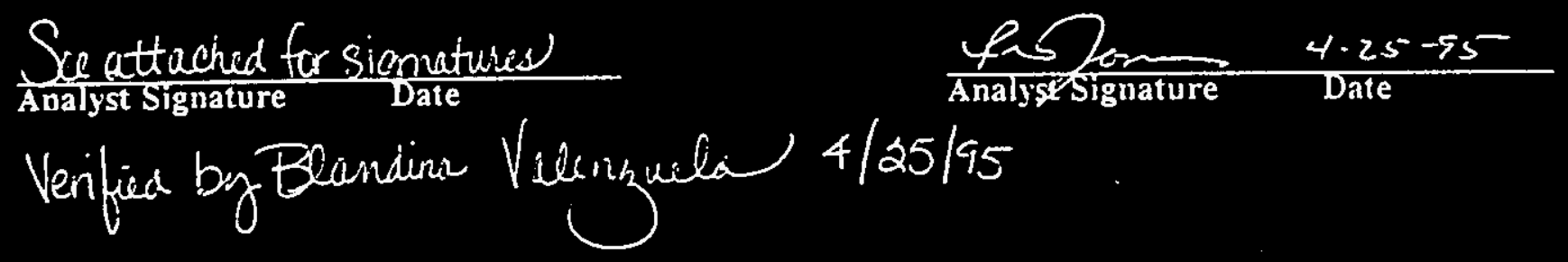

Dara Enry Comments: The DuP2 for Samples S957000357 and 5957000452 uere

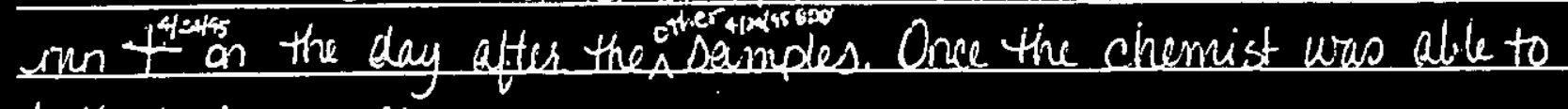
lok at the results.

Units shown for QC (SPK \& STD) may not reflect the actual units. $D L=$ Detection Limit, $S=$ Worklist Slor Number, $R=$ Replicare Number, $A=$ Aliquot Code. 


\section{LABCORE Data Entry Template for Worklist\# 929}

Analyst: $\quad$ ews $\quad$ Instrument: TGA01 Book $\#-42 k 872$

Method: LA-560-112 Rev/Mod Af-2

Worklist Comment: Please run B-112 TGA under N2. bdv

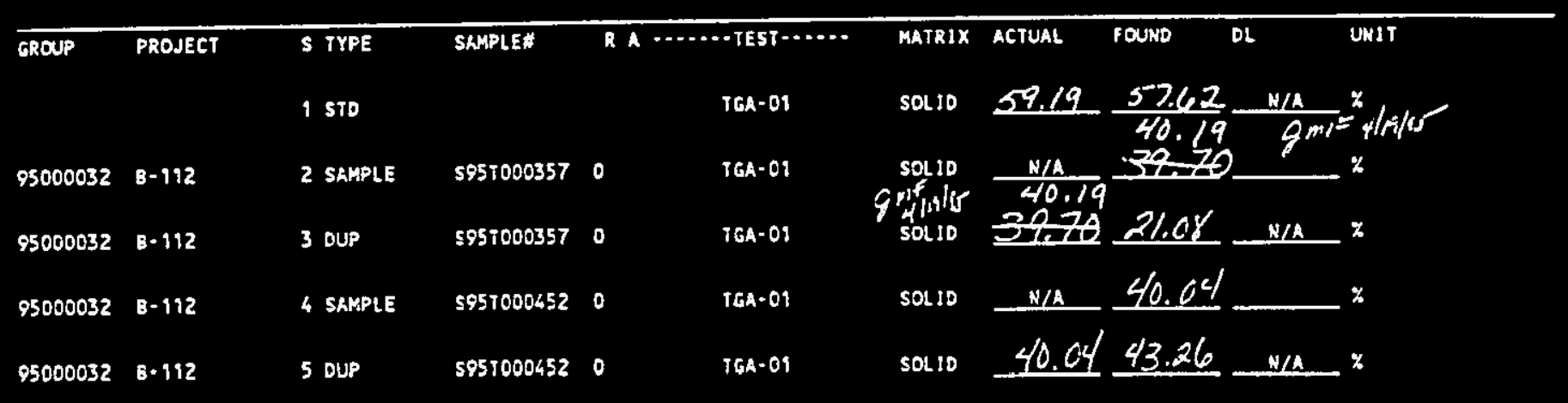

\section{Final page for worklist \# 929}
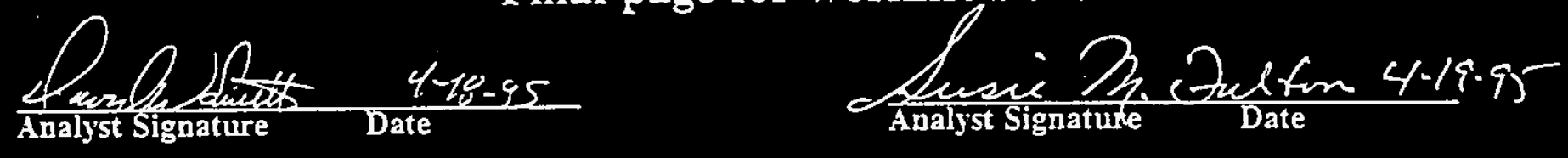

Dara Entry Comments:

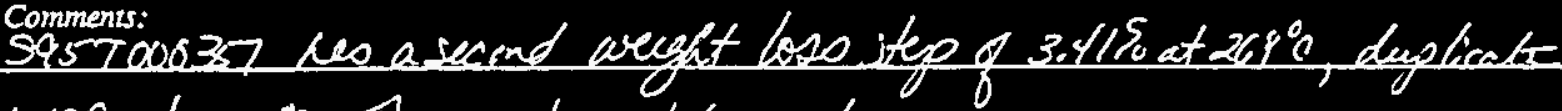

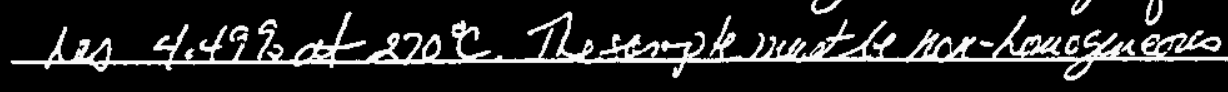

Units shown for QC (SPK \& STD) may not-reflect the actual units. $D L=$ Detection Limit, $S=$ Worklist Slot Number, $R=$ Replicate Number, $A=$ Aliquot Code. 


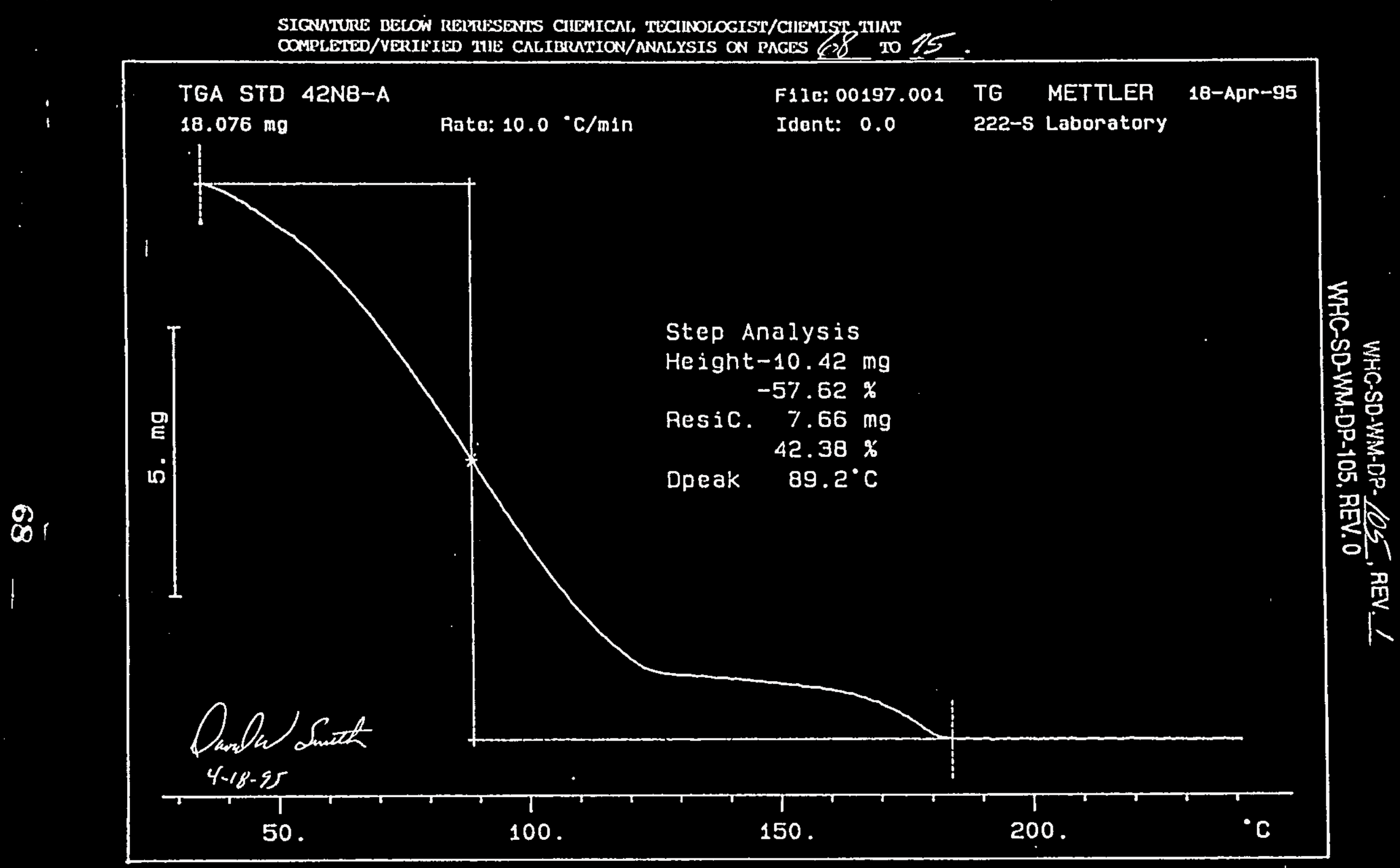




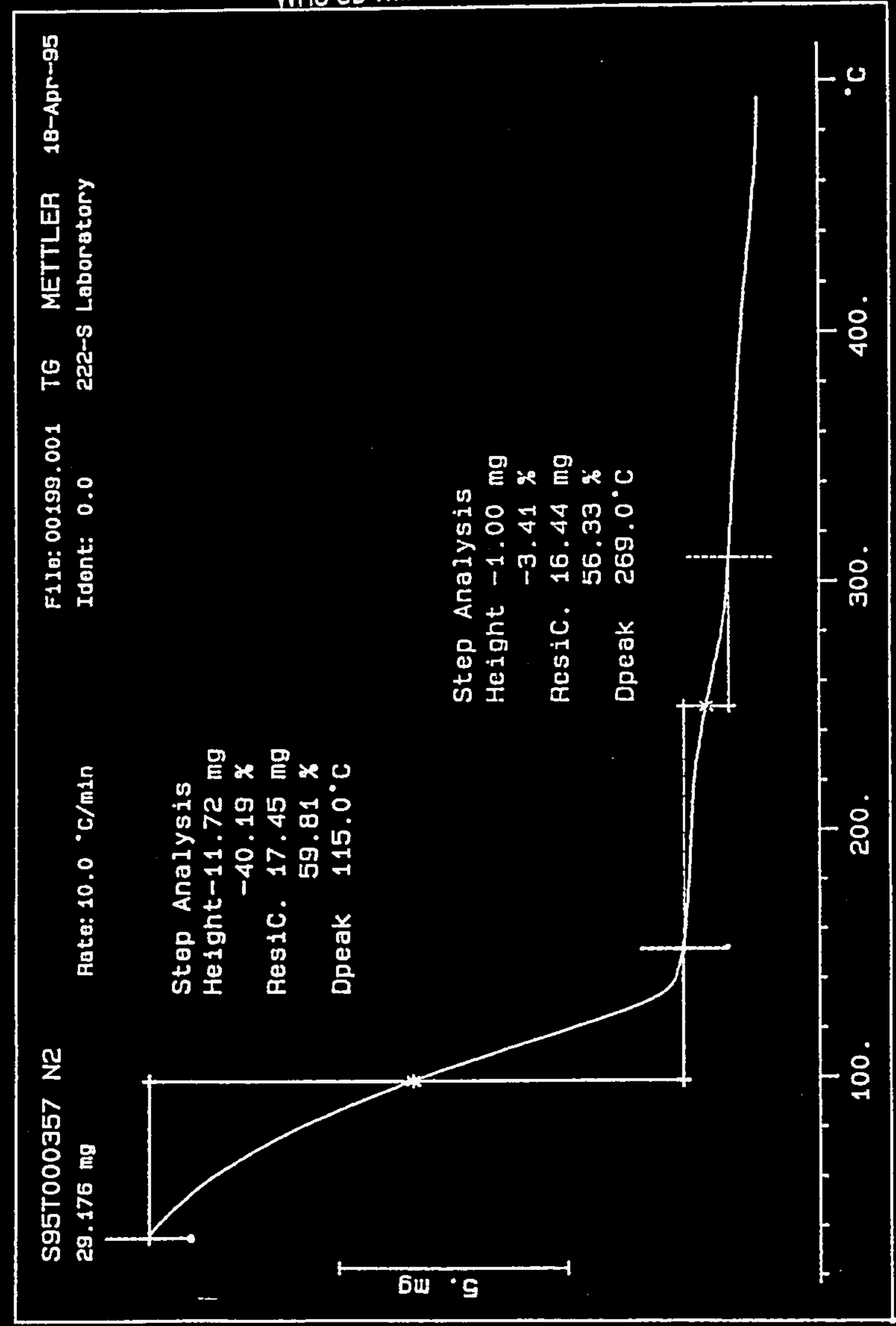




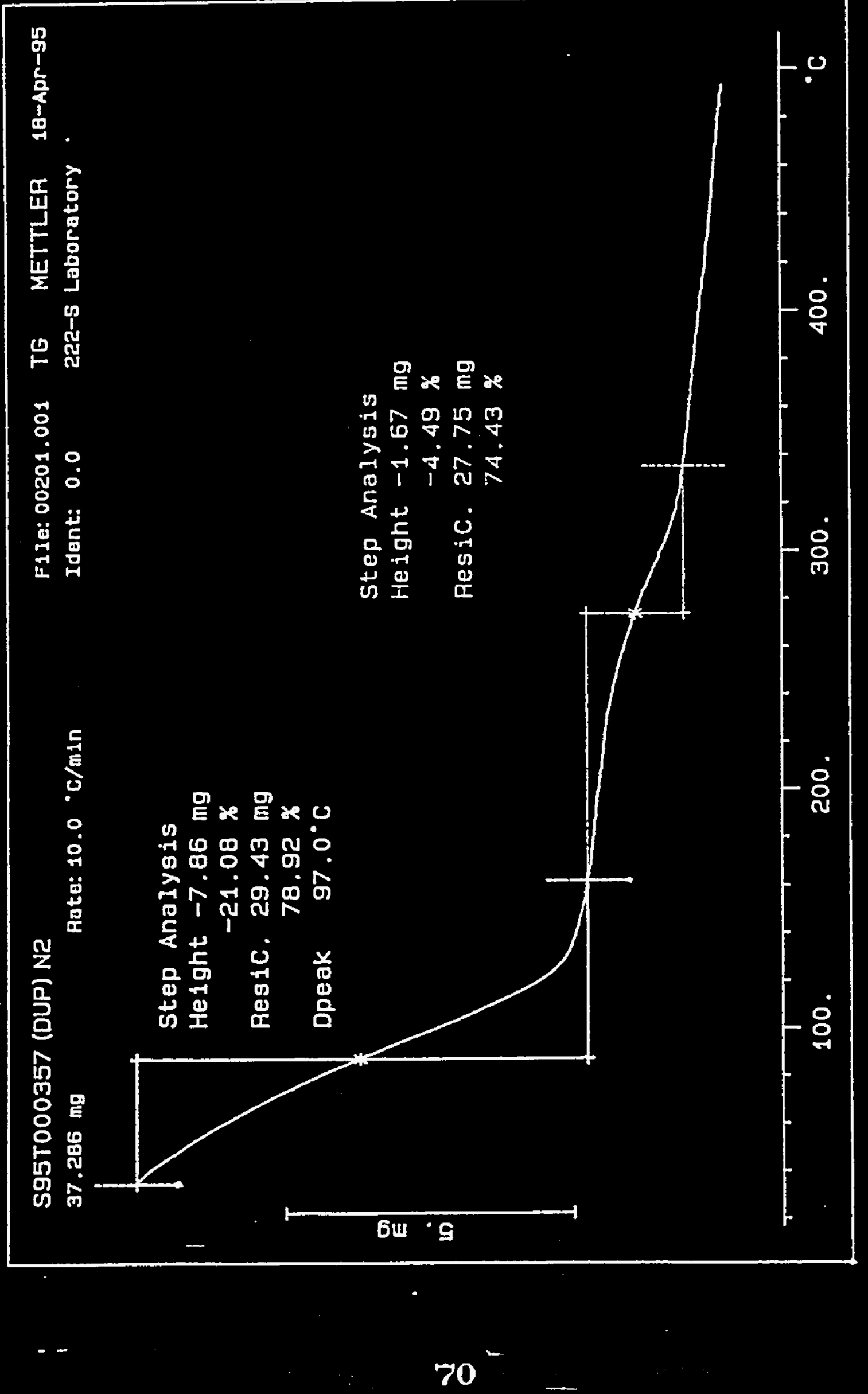




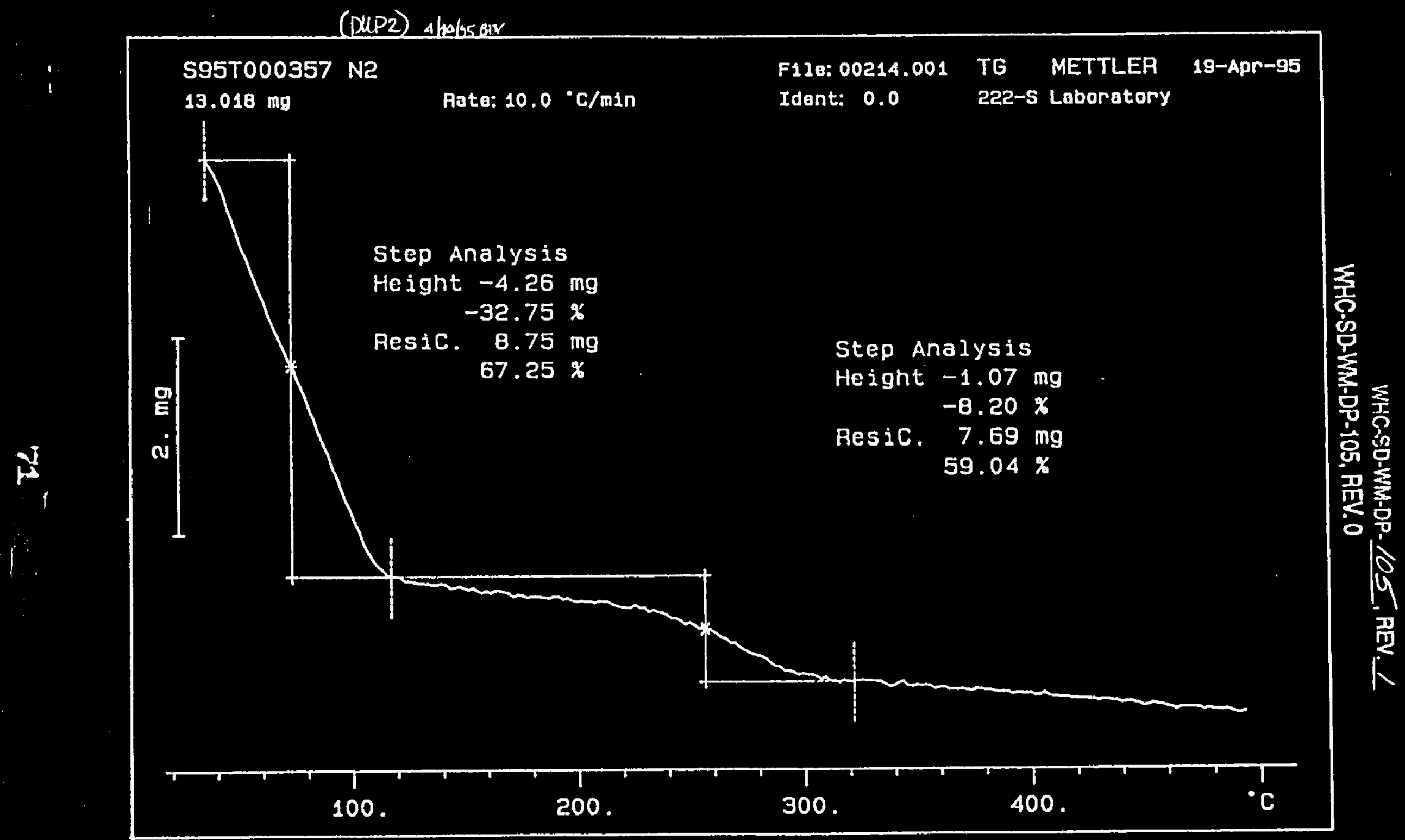




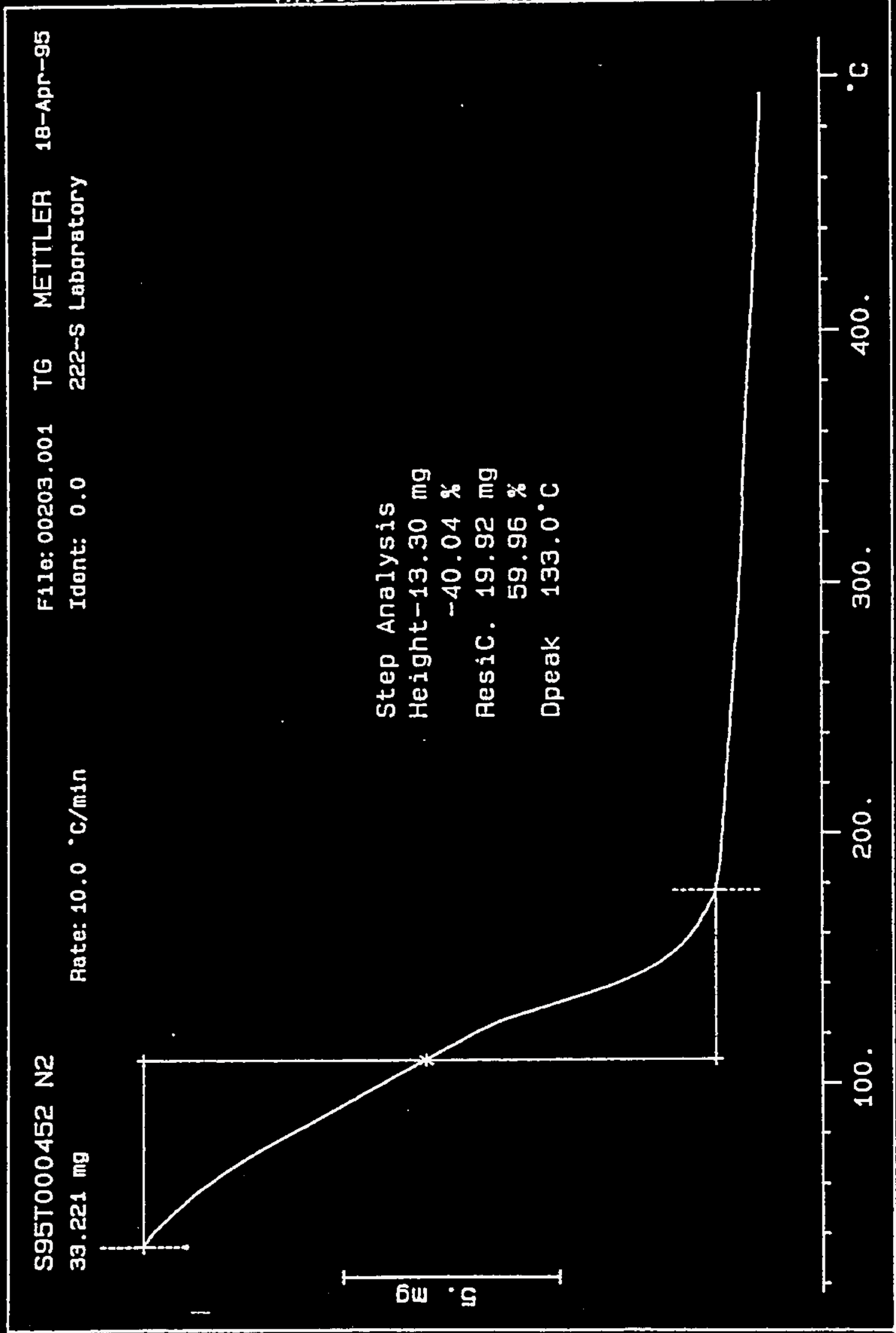




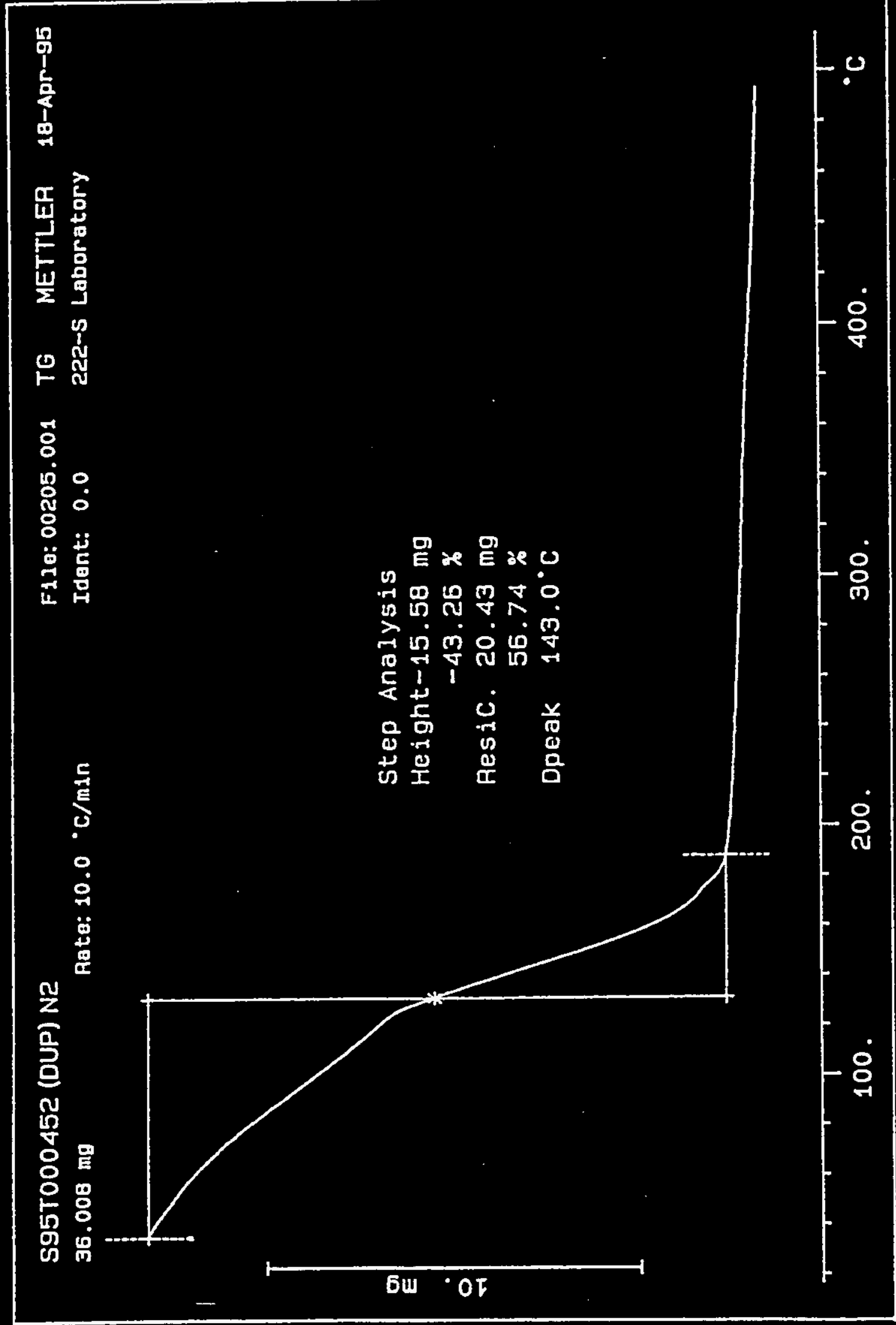




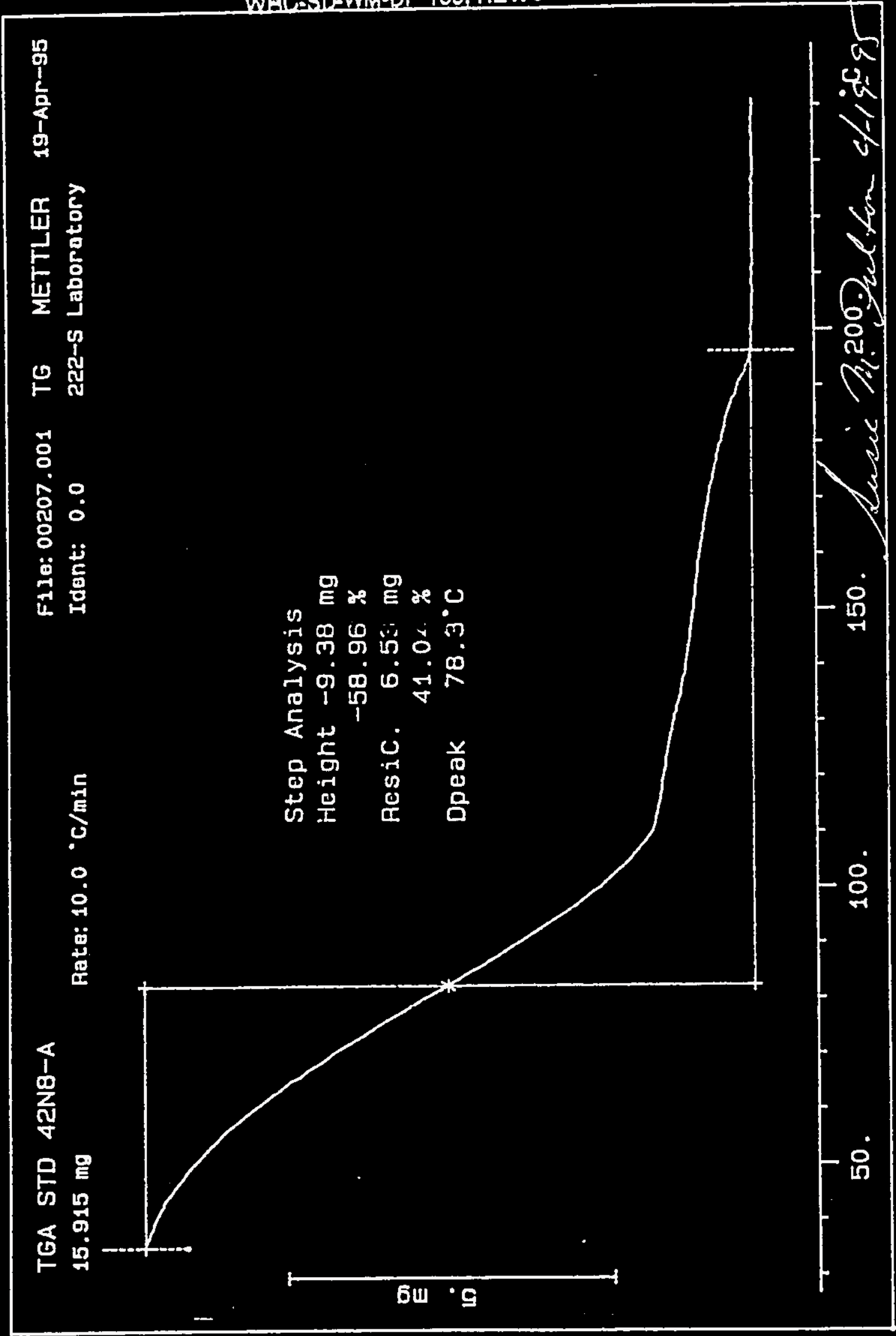




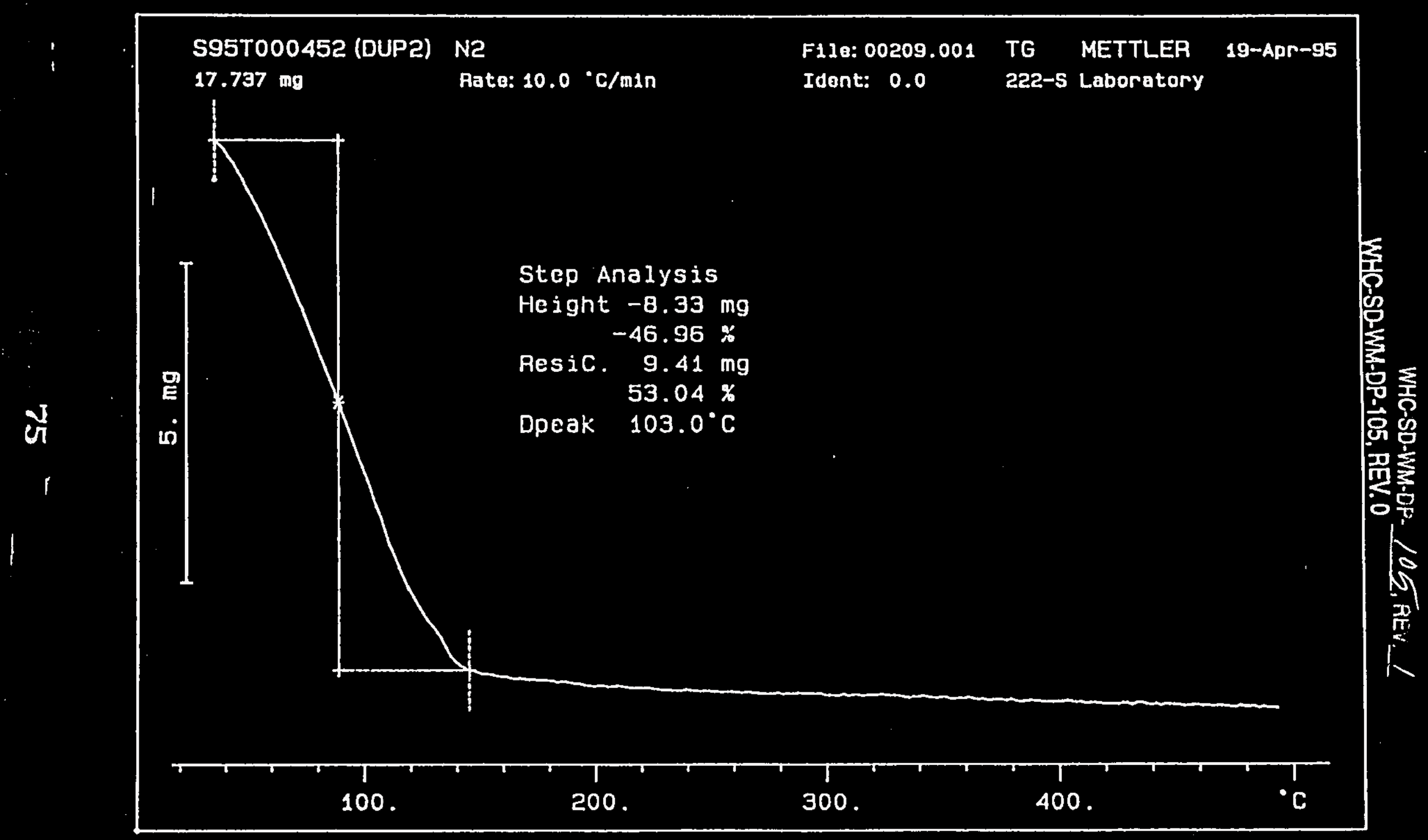


WHC-SD-WM-DP-105, REV.L.

worklistrpt Version 2.002/21/95

\section{WHC-SD-WM-DP-105, REV. 0
LABCORE Data Entry Template for Worklist\# 930}

Page: I

Analyst: $\quad$ ENE Instrument: TGA01 Book $42 N 8-A$

Method: LA-560-112 Rev/Mod A. 2

IVorklist Comment: Please run B-112 TGA under N2. bdv

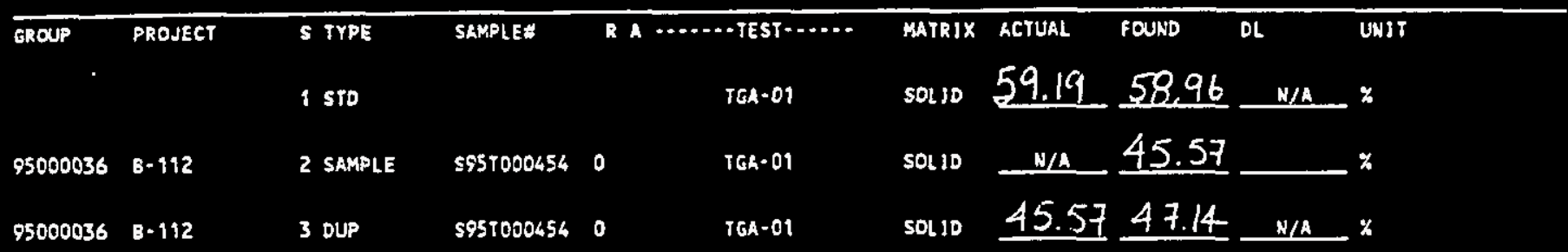

\section{Final page for worklist \# 930}

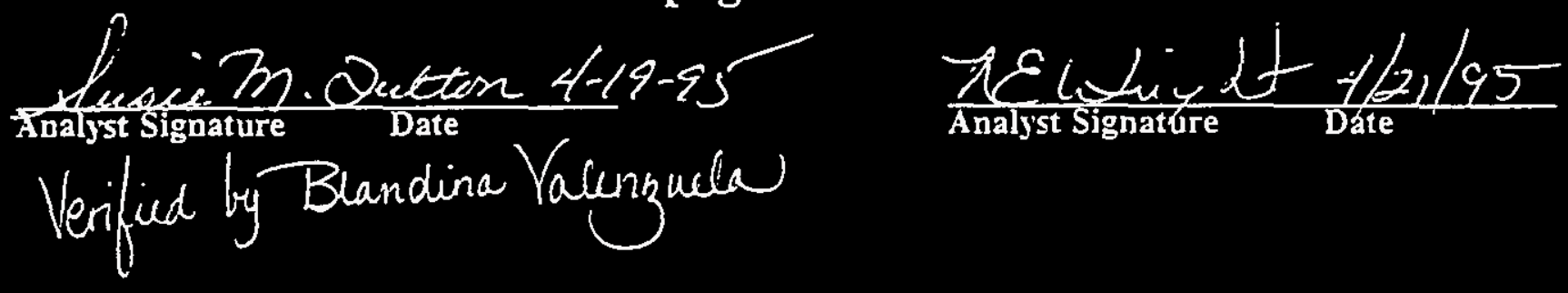

Data Entry Comments:

Units shown for QC (SPK \& STD) may not rglect the actual units. $D L=$ Detection Limit, $S=$ Worklist Slot Number, $R=$ Replicate Number, $A=$ Aliquot Code. 
WHC-SD-WM-DP-105, REV. 0

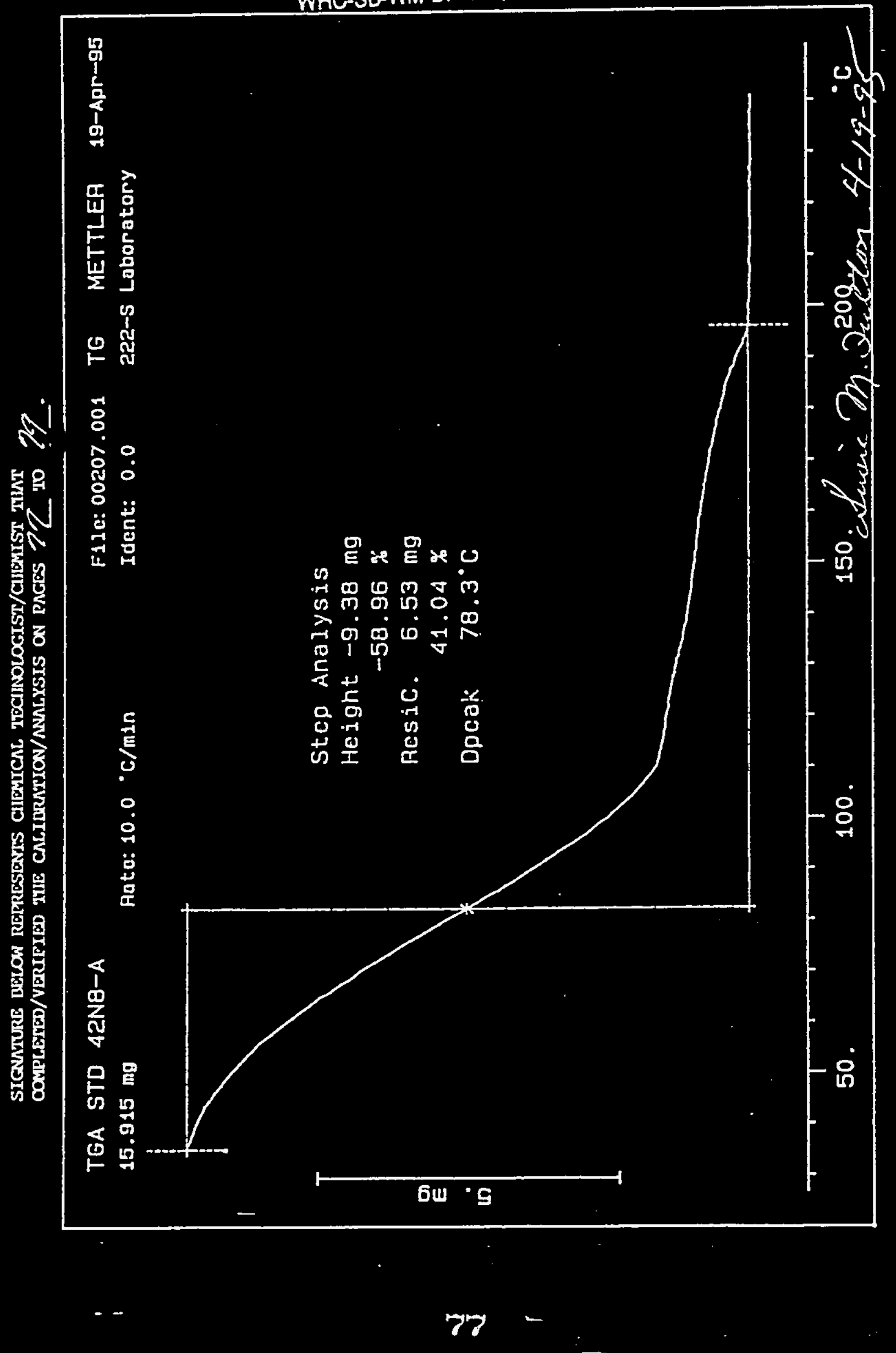


WHC-SD-WM-CP-105, REV. I

WHC-SD-WM-DP-105, REV. O

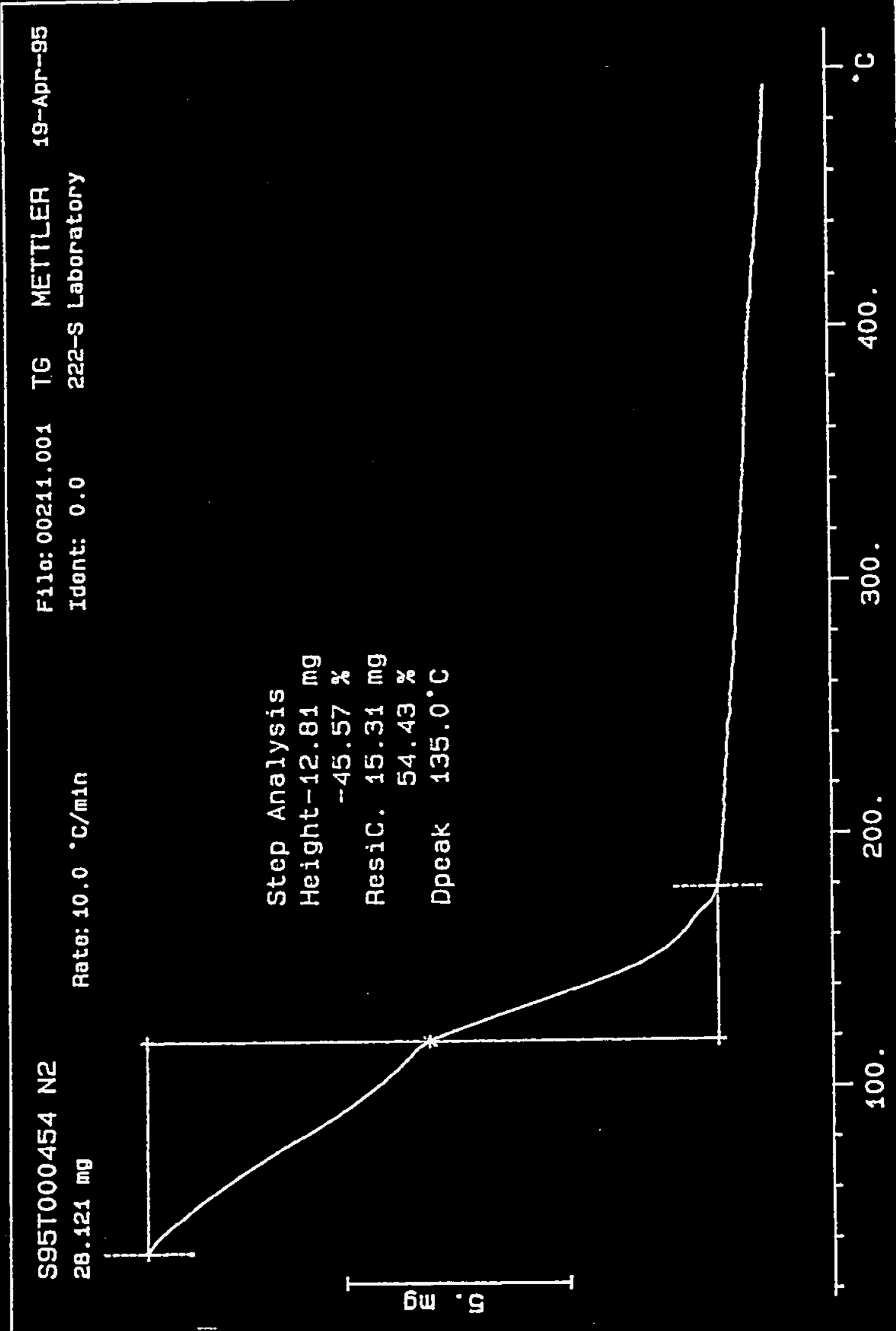

$\cdots$ 


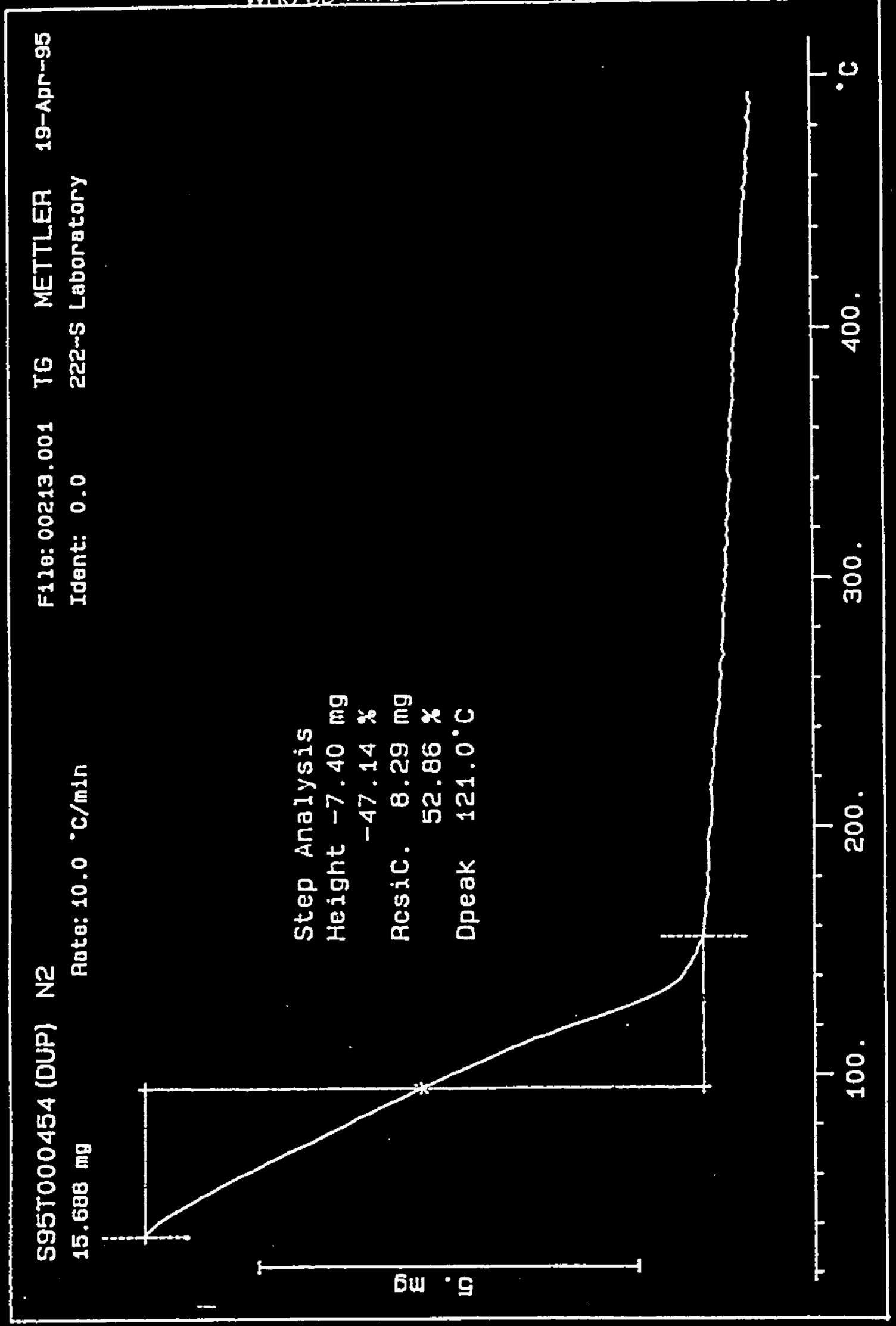

79 